\title{
Cassini Exploration of the Planet Saturn: A Comprehensive Review
}

\author{
Andrew P. Ingersoll ${ }^{1}$
}

Received: 10 October 2019 / Accepted: 10 October 2020 / Published online: 26 October 2020

(C) The Author(s) 2020

\begin{abstract}
Before Cassini, scientists viewed Saturn's unique features only from Earth and from three spacecraft flying by. During more than a decade orbiting the gas giant, Cassini studied the planet from its interior to the top of the atmosphere. It observed the changing seasons, provided up-close observations of Saturn's exotic storms and jet streams, and heard Saturn's lightning, which cannot be detected from Earth. During the Grand Finale orbits, it dove through the gap between the planet and its rings and gathered valuable data on Saturn's interior structure and rotation. Key discoveries and events include: watching the eruption of a planet-encircling storm, which is a 20- or 30-year event, detection of gravity perturbations from winds $9000 \mathrm{~km}$ below the tops of the clouds, demonstration that eddies are supplying energy to the zonal jets, which are remarkably steady over the 25-year interval since the Voyager encounters, re-discovery of the north polar hexagon after 25 years, determination of elemental abundance ratios $\mathrm{He} / \mathrm{H}, \mathrm{C} / \mathrm{H}, \mathrm{N} / \mathrm{H}, \mathrm{P} / \mathrm{H}$, and $\mathrm{As} / \mathrm{H}$, which are clues to planet formation and evolution, characterization of the semiannual oscillation of the equatorial stratosphere, documentation of the mysteriously high temperatures of the thermosphere outside the auroral zone, and seeing the strange intermittency of lightning, which typically ceases to exist on the planet between outbursts every 1-2 years. These results and results from the Jupiter flyby are all discussed in this review.
\end{abstract}

Keywords Saturn · Jupiter · Cassini $\cdot$ Atmosphere $\cdot$ Interior $\cdot$ Giant planet

\section{Introduction}

The planets themselves, Saturn and Jupiter, were an important scientific objective of the Cassini mission, and they are the focus of this review. The review is taken from Volume 1 of the Cassini Final Report (https://pds-rings.seti.org/cassini/). That document contains instrument-specific chapters, other discipline chapters beside the planets themselves, and a

\footnotetext{
A.P. Ingersoll

api@gps.caltech.edu

1 Division of Geological and Planetary Sciences, California Institute of Technology, 1200 East

California Blvd, Pasadena, CA 91125, USA
} 
science summary with a science assessment for the complete mission. For setting priorities and planning, Cassini had five discipline working groups: Icy satellites, magnetosphere, rings, Saturn, and Titan. The report is taken from the Saturn chapter. The overlap region, where the upper atmosphere of the planet merges with the near-vacuum of space, is covered in the magnetosphere chapter if charged particles are the dominant medium, and in the Saturn chapter if neutral gases are the dominant medium

The focus is on Cassini and the discoveries it made. The report is not a complete review of giant planet atmospheres and interiors. The reference list was compiled from the Cassini instrument reports and from literature searches with keywords Cassini, Saturn OR Jupiter, atmosphere OR interior but NOT magnetosphere, rings, Titan, or other satellites. I was selective with keywords words like ionosphere and aurora. I was also selective with models and laboratory work that didn't explicitly use Cassini data. I read abstracts and tried to paraphrase the most important results, but I downloaded and read only about $20 \%$ of the papers. The final literature search was done in mid-July of 2020.

Science results are in the first 9 sections of the paper: 1. Introduction. 2. Clouds, composition and chemistry. 3. Heat flux, winds and temperatures. 4. The great storm of 2010-2011. 5. Vortices, mean flow and eddies. 6. Interior structure and rotation. 7. Upper atmosphere physics and chemistry. 8. Jupiter science results. 9. Venus atmosphere. Section 10 gives the open science questions after Cassini. Section 11 gives the Saturn portion of the scientific objectives for the prime and solstice missions. The latter was a mission extension that allowed Cassini to observe all four seasons-summer and fall in the south and winter and spring in the north. The scientific objectives are quite general, but that's as it should be. Cassini was the first Saturn orbiter, and it was an extremely well-equipped spacecraft. It was a flagship sailing into unknown waters, and it was impossible to limit the objectives to a specific list drawn up in advance. Cassini was on a voyage of discovery, and it was wildly successful because there was much to be discovered.

\section{Clouds, Composition and Chemistry}

\subsection{Clouds and Haze}

The temperature field, cloud properties, and composition are an interacting system that controls the energy budget of the planet and the clouds and haze that we see in the planet's atmosphere (Fig. 1). Clouds are a tracer of the motion. They form on updrafts, which are part of the energy cycle that controls the zonal winds. Also, clouds interact with the temperature field through condensation and evaporation. Cassini, with its comprehensive array of instruments and unique perspective from different positions in the orbit, has contributed critical data on all of these objectives. In addition, Cassini has spawned exploration of Saturn from Earth-based telescopes.

Timed with the start of Cassini's exploration of the Saturn system, Beebe (2005) presented the case for comparative study of the outer planets. Papers on the Cassini instruments and their ability to measure clouds and haze include: Baines et al. (2005) on the Visual and Infrared Mapping Spectrometer (VIMS), Porco et al. (2005) on the Imaging Science Subsystem (ISS); Flasar et al. (2004, 2005) and Jennings et al. (2017) on the Composite Infrared spectrometer (CIRS); and Janssen et al. (2013) on the Cassini RADAR radiometer. Schinder et al. (2015) give the techniques for probing the electron density and temperature structure with radio occultations.

Banfield et al. (1998) describe the use of near-infrared (IR) spectroscopy from the 5-m Hale telescope at Palomar, California to measure the altitudes and scattering properties of 
Fig. 1 Image taken by the ISS in 2004 as Cassini was approaching Saturn. It was summer in the south, and the sunlight was driving a rich hydrocarbon chemistry. Temperatures were $10-20 \mathrm{~K}$ warmer in the south. The north was mostly shielded from sunlight, and the skies were blue, as shown by the sliver of atmosphere visible above the ring (PIA05389)

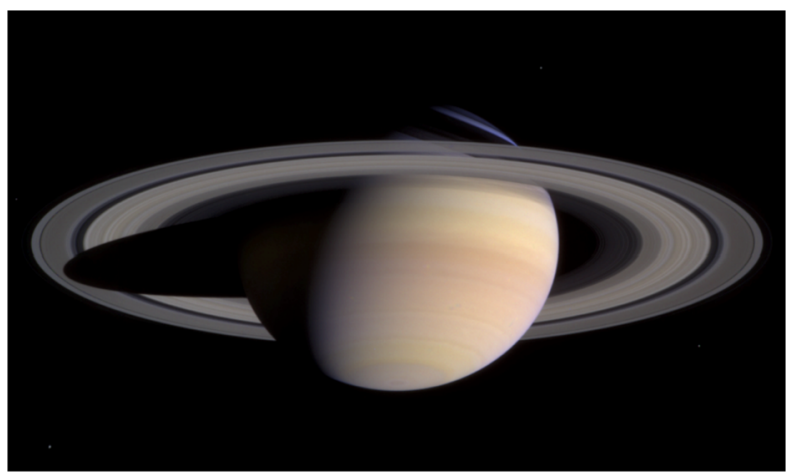

clouds and haze. Similarly, Sanchez-Lavega et al. (2004) described Hubble Space Telescope observations of cloud morphology in Saturn's atmosphere. Temma et al. (2005) used Earthbased telescopic observations to probe the vertical structure of Saturn's cloud layers. Using Hubble Space Telescope (HST) data, Perez-Hoyos et al. (2006) documented short-term variability (weeks to months) associated with cloud reflectivity of the belts and zones. West et al. (2009) gives a comprehensive survey of clouds and haze in Saturn's atmosphere as inferred from Cassini VIMS and ISS, as well as HST and other Earth-based telescopes. Generally cloud particles form by condensation of simple molecules, and haze particles form by condensation of complex molecules that are produced by photochemistry. Ingersoll et al. (2018) present images taken during the last few months of the mission at resolutions down to $0.5 \mathrm{~km}$ per pixel. The interesting features include filamentary clouds coherent over $20,000 \mathrm{~km}$ and puffy clouds resembling terrestrial cumulus. Spilker (2019) gives the highlights and discoveries obtained during Cassini's final year, including the some of the highest-resolution information about the interior and atmosphere.

Fletcher et al. (2007b) used Cassini CIRS data to probe the vertical and horizontal temperature structure and its relation to the belt and zone cloud structure. Roman et al. (2013) used ISS data to probe the layered cloud structure from the haze layers at 40 mbar to tropospheric clouds at 2.5 bar. Laraia et al. (2013) used Cassini RADAR radiometer data to probe the vertical distribution of $\mathrm{NH}_{3}$ to depths below its nominal cloud base. Barstow et al. (2016) use Cassini VIMS to document a photochemical origin for the stratospheric haze and either $\mathrm{NH}_{3}$ or $\mathrm{NH}_{4} \mathrm{SH}$ for the deeper tropospheric cloud. Using Cassini ISS data, Dyudina et al. (2016) and Perez-Hoyos et al. (2016) quantified the way the atmosphere scatters light, including the phase function and the spherical and Bond albedos.

Del Genio et al. (2009) give a comprehensive review of Saturn's atmospheric structure and dynamics. The early data come from the Voyager flybys and Earth-based telescopes and then from the remote-sensing instruments on Cassini. The review covers the deep atmosphere, the troposphere, the stratosphere, and dynamical features such as the jets, waves, discrete features. Del Genio and Barbara (2016) provide a classification scheme based on cloud morphology for revealing the underlying dynamics.

\subsection{Elemental and Isotopic Abundances}

Counted by the number of atoms, the Sun is approximately $99.9 \%$ hydrogen/helium and $0.1 \%$ everything else. In our solar system, Jupiter comes closest to having the same abundance ratios relative to $\mathrm{H}$ as the Sun and Saturn comes next, with higher heavy-element ratios than Jupiter. Abundance ratios relative to $\mathrm{H}$ that are different from the solar ratio provide observational constraints for the formation and evolution of the planet. 
The helium to hydrogen ratio in the atmosphere reflects the value in the solar nebula when the planets were forming as well as the degree to which helium has settled toward the core. Settling is thought to happen because helium becomes immiscible in metallic hydrogen, which is the dominant constituent at megabar pressures. The settling releases gravitational potential energy, which affects the cooling history and planetary evolution. In principle, helium is detected by remote sensing through its effect on the molecular weight of the gas. One uses occultation data to get density vs. altitude, and from the hydrostatic equation one gets density vs. pressure. Then one uses infrared data to get temperature vs. pressure, and with the equation of state one can solve for the molecular weight. The method was used for Jupiter and Saturn using Voyager data (Conrath et al. 1984), but the Galileo probe gave a different result for Jupiter and forced a reanalysis for Saturn (Conrath and Gautier 2000). Cassini has collected excellent data using CIRS for the temperature and occultations by several instruments—radio science, VIMS, and the Ultraviolet Imaging Spectrograph (UVIS), but getting a consistent estimate of the helium abundance is difficult. The occultation result depends on the ray path, and that depends on unknown winds and temperatures above the occultation point. The most recent estimate (Koskinen and Guerlet 2018) uses Cassini UVIS for the occultations and CIRS for temperature versus pressure. They get a helium mass fraction $\mathrm{Y}$ of $0.16-0.22$, which is about $70 \%$ of the protosolar mass fraction of 0.27 , implying that a certain amount of settling has occurred in Saturn's interior.

Before Cassini reached Saturn, the $\mathrm{C} / \mathrm{H}$ abundance ratio was known to be higher on Saturn than on Jupiter, which was known to be higher than that on the Sun (Atreya et al. 1999, 2003; Owen and Encrenaz 2003). The C/H ratio is perhaps the most accurately known ratio of all the heavy elements because its molecular form, methane, doesn't condense at Saturn temperatures and isn't readily destroyed in chemical reactions. Cassini CIRS probes the far-infrared rotational lines of the molecule and has determined a volume mixing ratio of $(4.7 \pm 0.2) \times 10^{-3}$, corresponding to a $\mathrm{C} / \mathrm{H}$ enrichment relative to solar of 10.4 (Fletcher et al. 2009b). This is more than a factor of 2 greater than that for Jupiter but consistent with conventional models of the formation and evolution of the giant planets, which predict increasing ratios to $\mathrm{H}$ of the heavy element abundances relative to the Sun from Jupiter to Neptune (Atreya et al. 2019).

Saturn's greater enrichment is also qualitatively consistent with the masses and radii of the two giant planets. These, coupled with the gravitational moments, rotation rate, and an equation of state, give information about the core mass and amount of heavy elements in the molecular envelope (Guillot 1999; Nettelmann et al. 2013). Chemical models preceding the arrival of Galileo and Cassini listed many gases that were potentially observable in the atmospheres of Jupiter and Saturn (Fegley and Lodders 1994). Water is crucial because O is the third most abundant element after $\mathrm{H}$ and $\mathrm{He}$, but the vapor pressure is so low at the tops of the clouds that Cassini could not detect it. Methane is much more volatile than water, so it does not condense in Saturn's atmosphere. Thus the $\mathrm{C} / \mathrm{H}$ ratio derived from methane is likely to be representative of the planet as a whole.

The high $\mathrm{C} / \mathrm{H}$ ratio is taken as evidence of heavy element enrichment in general and has implications for possible differential sedimentation in the planet's interior over its lifetime (Mousis et al. 2006). Measurements of $\mathrm{NH}_{3}, \mathrm{PH}_{3}, \mathrm{C}_{2} \mathrm{H}_{2}, \mathrm{C}_{2} \mathrm{H}_{6}$, and $\mathrm{CH}_{4}$ all are consistent with these gases trapped as clathrate hydrate in the feeding zone of Saturn as the planets were forming (Hersant et al. 2008). Clathrate hydrates require water, and our lack of knowledge of the oxygen abundance allows for a number of interior models with a range of values for the C/O ratio (Lunine 2011). CIRS measurements suggest that Saturn's D/H ratio is less than that for Jupiter, which mostly reminds us that our understanding planetary formation and evolution is incomplete (Pierel et al. 2017). 

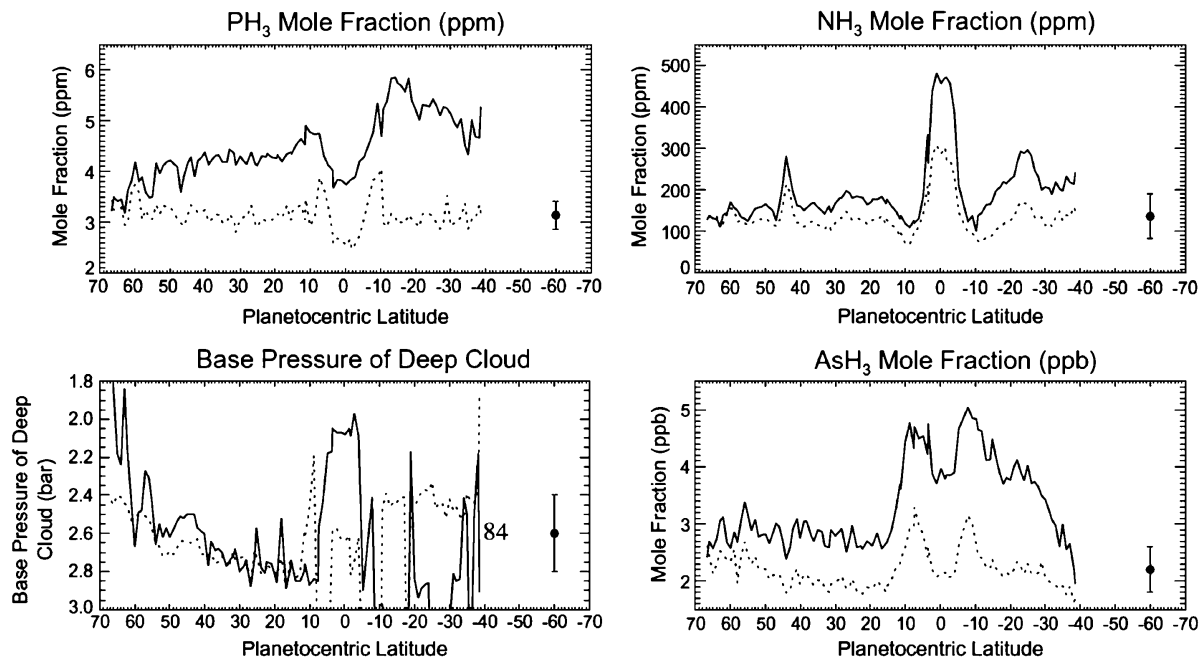

Fig. 2 Tropospheric chemical abundances from Cassini VIMS. On first look, the profiles do not tell a consistent story. $\mathrm{NH}_{3}$ shows a high mole fraction at the equator, consistent with upwelling there and downwelling on either side at $10-12^{\circ}$ latitude, but $\mathrm{PH}_{3}$ and $\mathrm{AsH}_{3}$ show low abundances at the equator, consistent with downwelling. The data may be indicating a double Hadley cell, with rising motion at the higher altitudes at the equator and sinking motion deeper down (Fletcher et al. 2011a)

Although ammonia is the main carrier of nitrogen on Jupiter and Saturn, the isotopic ratio ${ }^{15} \mathrm{~N} /{ }^{14} \mathrm{~N}$ can reveal insights into the molecular carrier $\left(\mathrm{N}_{2}\right.$ versus $\left.\mathrm{NH}_{3}\right)$ in the protoplanetary nebula. The Saturnian and Jovian isotopic ratios appear indistinguishable (Abbas et al. 2004; Fletcher et al. 2014; Fouchet et al. 2004a) and distinctly lower in ${ }^{15} \mathrm{~N}$ than Titan. That result favors accretion of primordial $\mathrm{N}_{2}$ on both planets, either in the gas phase from the solar nebula or as ices formed at very low temperature (Fletcher et al. 2014), and a different source reservoir for the ${ }^{15} \mathrm{~N}$-enriched atmosphere of Titan.

\subsection{Variability of Trace Gases}

The gaseous composition of a giant planet atmosphere varies due to several processes: condensation and precipitation that remove the particles from the gas, updrafts and downdrafts that alter the chemical equilibrium of the various reacting species, and photochemical reactions that lead to dissociation and/or ionization. In addition, the upper atmosphere may receive molecules and solid particles from the rings and satellites.

Fletcher et al. (2011a) review the elements that appear in simple compounds that are detectable in VIMS spectra, including $\mathrm{PH}_{3}, \mathrm{NH}_{3}, \mathrm{AsH}_{3}, \mathrm{GeH}_{3}, \mathrm{CO}, \mathrm{CH}_{3} \mathrm{D}$, and ${ }^{13} \mathrm{CH}_{4}$. They use VIMS 4.6-5.1 $\mu \mathrm{m}$ nightside spectra and solve for cloud properties and molecular abundances in the 1-4 bar pressure range. Figure 2 is the result, and there seem to be contradictions in whether there is upwelling or downwelling at the equator. $\mathrm{NH}_{3}$ is removed from the gas by condensation and precipitation, but only above the 1.5 bar level. $\mathrm{PH}_{3}$ and $\mathrm{AsH}_{3}$ are removed from the gas by chemical reactions at pressures $\leq 3$ bars. The VIMS is seeing variability at different altitudes. Fletcher et al. calculate that the maximum of $\mathrm{NH}_{3}$ at the equator indicates equatorial upwelling at $\mathrm{P}<1.3$ bars, and the minima of $\mathrm{PH}_{3}$ and $\mathrm{AsH}_{3}$ at the equator indicate equatorial downwelling at $\mathrm{P}>2.8$ bars. In other words, Fletcher et al. are postulating two stacked, oppositely-directed Hadley cells based on their 
data. Clouds make the inversion non-unique, and the authors concede that the idea is speculative. The 2-cell idea originated with the Galileo mission to Jupiter (Ingersoll et al. 2000; Gierasch et al. 2000; Showman and de Pater 2005) to explain the preponderance of lightning in the (cyclonic) belts despite strong evidence of upwelling in the upper troposphere of the (anticyclonic) zones (Gierasch et al. 1986).

Fouchet et al. (2009) give a comprehensive review of Saturn composition and chemistry. Howett et al. (2007) and Guerlet et al. (2009) focus on $\mathrm{C}_{2} \mathrm{H}_{2}, \mathrm{C}_{2} \mathrm{H}_{6}$, and $\mathrm{C}_{3} \mathrm{H}_{8}$, which are produced above the $1 \mathrm{mbar}$ level by photodissociation of methane. They use Cassini CIRS data to infer a stratospheric circulation from south to north during the southern summer. Guerlet et al. (2010) added $\mathrm{C}_{3} \mathrm{H}_{8}$ and $\mathrm{CH}_{3} \mathrm{C}_{2} \mathrm{H}$ to the list of tracers and again found evidence of upwelling in the south and downwelling in the north. Fletcher et al. (2007a) also used CIRS data, focusing on phosphine, $\mathrm{PH}_{3}$. It is in chemical equilibrium below the cloud tops and is destroyed by photolysis in the stratosphere. The altitudes of sources and sinks are opposite to that of $\mathrm{C}_{2} \mathrm{H}_{2}$ and $\mathrm{C}_{2} \mathrm{H}_{6}$, but the inferred circulation is similar.

Cassini CIRS data stimulated photochemical modeling in order to quantify the inferred circulations (Dobrijevic et al. 2011; Hue et al. 2015). The data also stimulated laboratory measurements (Auwera et al. 2007; Devi et al. 2014) to refine the infrared absorptions of these gases. Gases like $\mathrm{CH}_{4}, \mathrm{C}_{2} \mathrm{H}_{2}$, and $\mathrm{C}_{2} \mathrm{H}_{6}$ absorb and emit in the infrared, and their spatial and temporal variations create temperature variations, which in turn affect the circulation. These interactions are studied with a 2-D photochemical model (Hue et al. 2016). They obtain "moderate" agreement with the observed species distributions, but they say that more sophisticated 2D and 3D representations of transport are needed.

Intensified Earth-based observations are a further desirable result of Cassini's mission to Saturn. Some of the observations immediately preceded the outpouring of Cassini data (Kim and Geballe 2005; Tejfel 2005), both to extend the range of temporal variability and to compare inferences based on different data sets. For instance, microwaves are sensitive to ammonia vapor at deeper levels than those probed by infrared radiation. The Cassini RADAR used in passive mode collects thermal radiation at $2.2 \mathrm{~cm}$ wavelength. With laboratory measurements of the ammonia opacity (Hanley et al. 2009), the Cassini $2.2 \mathrm{~cm}$ data were used to infer depletion of ammonia below the expected base of the ammonia cloud (Janssen et al. 2013; Laraia et al. 2013). This depletion is observed on Jupiter as well and has not been fully explained. The $\mathrm{NH}_{3}$ abundance is controlled mostly by condensation and evaporation, which is more tied to the cloud structure, so it reveals different aspects of the circulation (Hurley et al. 2012b) than that of the hydrocarbons.

In giant planet atmospheres, the principal oxygen-bearing molecules are $\mathrm{H}_{2} \mathrm{O}$ and $\mathrm{CO}$, with the latter in equilibrium at higher temperatures and pressures than the former. However, water is frozen out at high altitudes, so the presence of $\mathrm{CO}_{2}$ at the 1 mbar level suggests a source outside the planet. Abbas et al. (2013) argued that the oxygen source is interstellar dust. Cavalie et al. (2019) argued that the plumes of Enceladus are the most likely source. Abbas et al. use Cassini CIRS data and Cavalie et al. use Hershel data, but the latter group base their argument on the fact that stratospheric water peaks at the equator whereas interstellar dust would not.

\section{Heat Flux, Winds and Temperatures}

\subsection{Radiation Budget}

The internal heat coming out of the planet is a fundamental quantity relevant to formation and evolution. Because giant planets in our solar system are massive and their surfaces are 
Fig. 3 Thermal emission of the planet in units of $\mathrm{W} \mathrm{m}^{-2}$ from Cassini CIRS. In the south the season was early summer in 2004 and mid fall in 2013. The decrease in thermal emission in the south was due to cooling of the atmosphere. Comparing the years before and after 2011, it is clear that the great storm increased the thermal emission by $5-10 \%$ in the $30-40^{\circ}$ latitude band, and that the anomaly persisted for several years $(\mathrm{Li}$ et al. 2015)

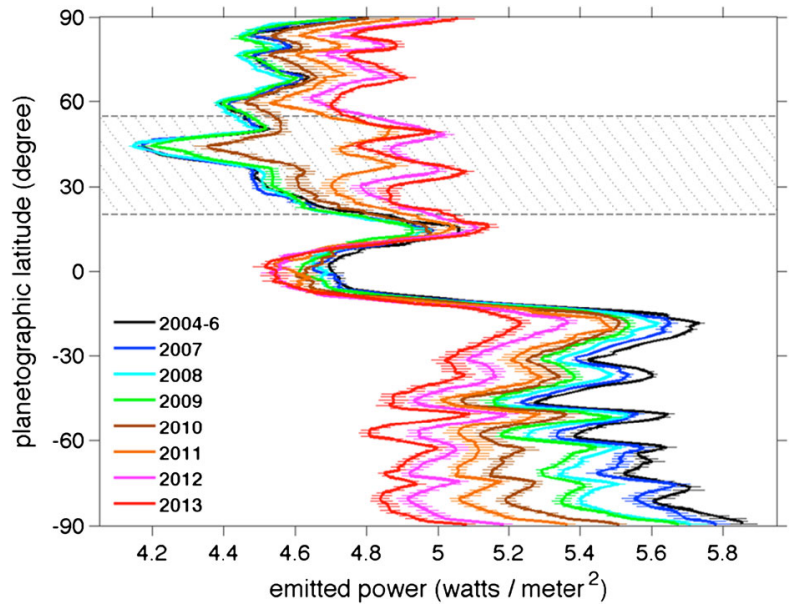

cold, they cool off slowly. Their interiors are still warm, and they are still releasing some of their heat of formation. To measure the internal power one takes the difference between the emitted infrared power and the absorbed solar power, which is the difference between the incident and reflected sunlight. Using Voyager data, Hanel et al. (1983) estimated the effective emission temperature as $95.0 \pm 0.4 \mathrm{~K}$, corresponding to an average emitted heat flux of $4.62 \pm 0.8 \mathrm{~W} \mathrm{~m}^{-2}$, and the ratio of emitted power to absorbed power as $1.78 \pm 0.09$. Thus the internal power divided by the absorbed solar power is 0.78 . Using data from Cassini CIRS, Li et al. (2010) estimated the emitted heat flux as $4.952 \pm 0.035 \mathrm{~W} \mathrm{~m}^{-2}$ during 2004 2009 , although it decreased by $2 \%$ during that time and was $16 \%$ higher in the southern hemisphere than in the northern hemisphere. These were likely seasonal changes associated with the approach of vernal equinox in 2009. Li et al. (2015) measured the emitted power vs. latitude from 2004 to 2013 and documented the 2010 great storm's effect, which was to increase the global emitted power by $2 \%$ and the power at the latitude of the storm by $9 \%$ (Fig. 3). These changes were in addition to a seasonal warming of the north and cooling of the south from 2004 to 2013.

\subsection{Seasonal Variations}

Two opposing effects control the amplitude of seasonal variations on Saturn (Fig. 4). The obliquity is $26.73^{\circ}$, which is larger than that of Earth, and is tending to produce large seasonal variations in incident sunlight. These variations are augmented by the rings, which block sunlight from reaching large parts of the winter hemisphere. On the other hand, Saturn's troposphere is massive, with clouds of water down to the 20-bar level, assuming the enrichment of water relative to solar composition is the same as that of methane, whose abundance can be measured spectroscopically. Further, the high specific heat of hydrogen gives the troposphere a huge thermal inertia, so the atmosphere tends to average over the seasonal cycle rather than respond to it. Seasonal averaging is reduced in the stratosphere (Fig. 4), because it has less thermal inertia than the troposphere. Seasonal change is reflected in the emitted power as a function of latitude ( $\mathrm{Li}$ et al. 2015) as the northern hemisphere season changed from winter to late spring over the period 2004-2013 (Fig. 3).

Using Cassini CIRS data Fletcher et al. (2010) showed that the stratosphere has a greater range of temperatures than the troposphere, which is consistent with the greater thermal 
(a) 2005

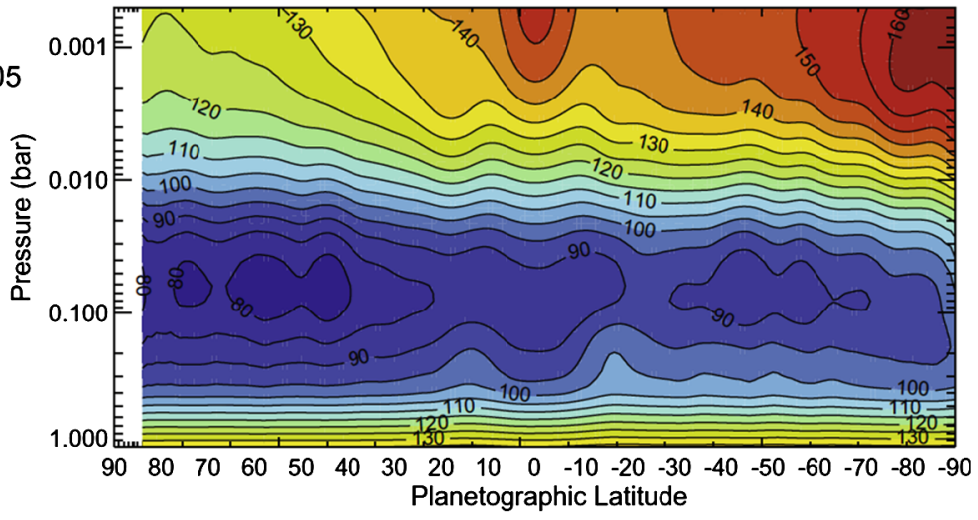

(b) 2008

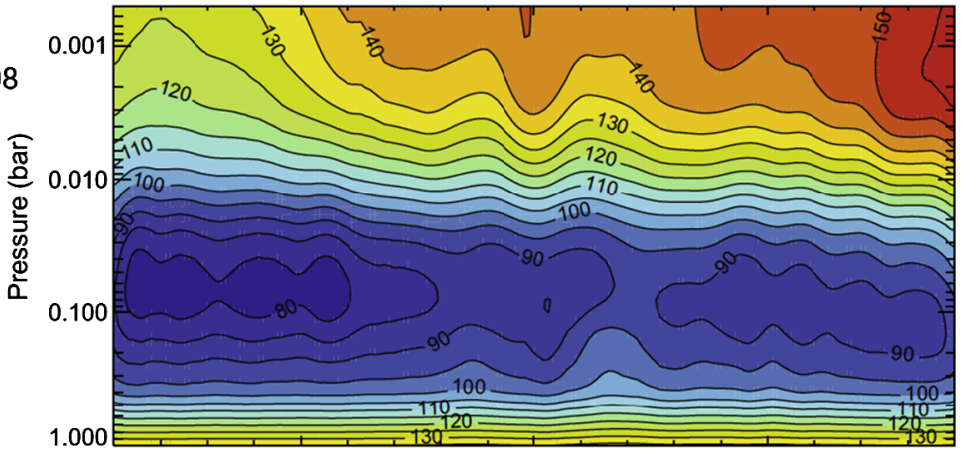

$\begin{array}{lllllllllllllllllll}90 & 80 & 70 & 60 & 50 & 40 & 30 & 20 & 10 & 0 & -10 & -20 & -30 & -40 & -50 & -60 & -70 & -80 & -90\end{array}$

Planetographic Latitude

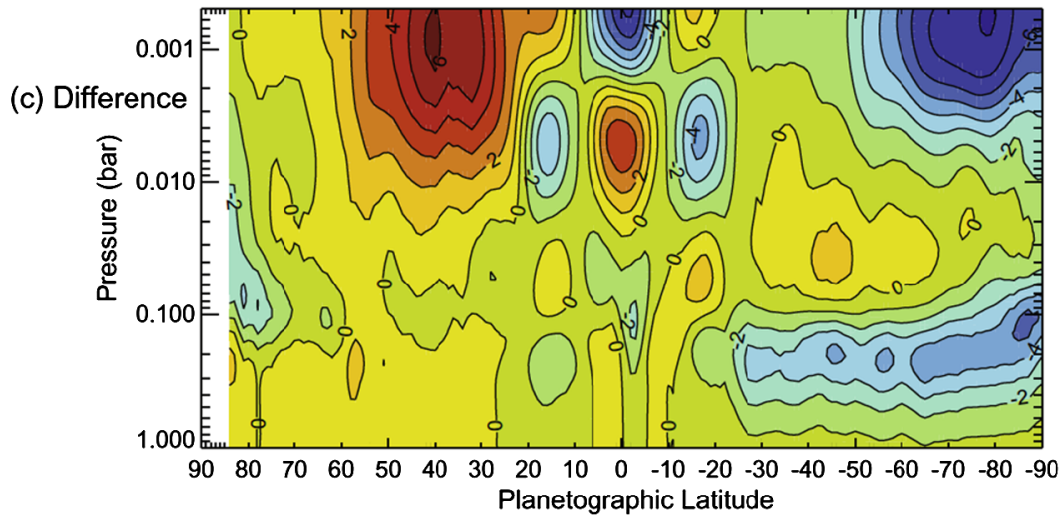

Fig. 4 Temperature as a function of latitude and altitude from Cassini CIRS. In 2005 the season was mid-winter in the north, and in 2008 the season was late winter. Vernal equinox was August 10, 2009. The south is warmer than the north in both years, although most of the hemispheric contrast and most of the difference between 2005 and 2008 is confined to high altitudes (Fletcher et al. 2010)

inertia of the latter. They point out that a photochemical haze develops in the spring/summer hemisphere (Fig. 1). Fletcher et al. (2015) observed polar cooling and depletion of acetylene in the southern hemisphere during fall and winter. Tropospheric temperature variations are 


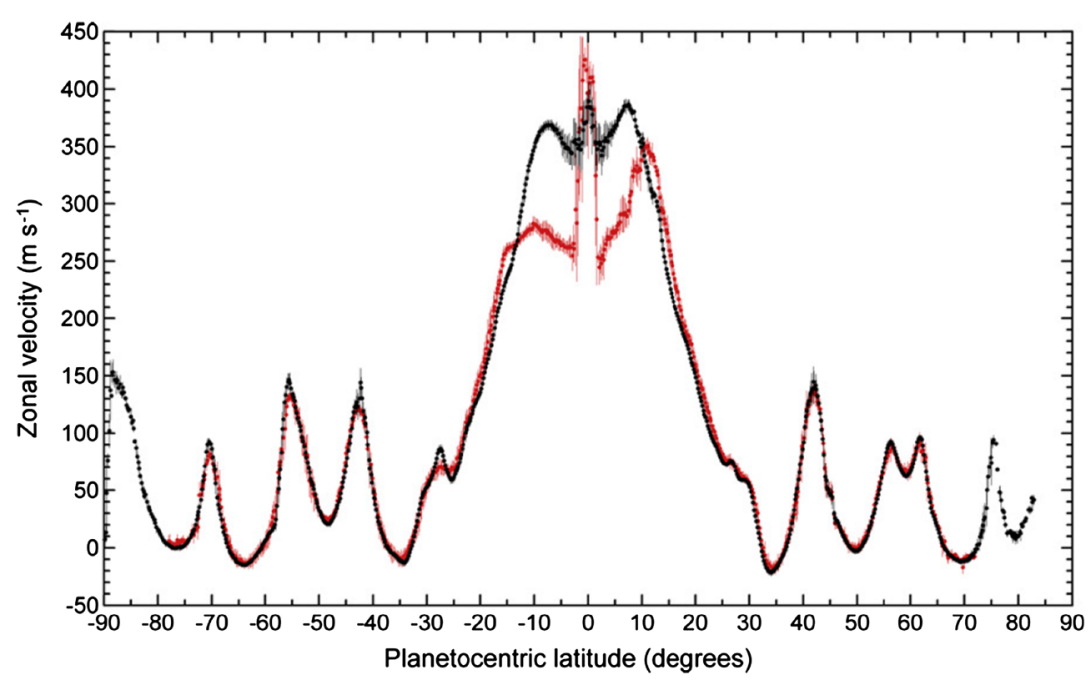

Fig. 5 Atmospheric zonal velocity for Saturn from Cassini ISS. The black curve is data from the 350 to 700 mbar levels, and the red curve is data from 100 to 200 mbar. The differences are small except for a latitude band $\pm 15^{\circ}$ from the equator (Garcia-Melendo et al. 2011b)

small (Fletcher et al. 2016) and show a substantial seasonal lag time, as expected. These seasonal changes are superposed on a general upwelling in the tropics and downwelling at the poles, as revealed by the two states (ortho and para) of the $\mathrm{H}_{2}$ molecule (Fletcher et al. 2016, 2017). Sinclair et al. (2013) and Sylvestre et al. (2015) compare CIRS observations of $\mathrm{C}_{2} \mathrm{H}_{2}, \mathrm{C}_{2} \mathrm{H}_{6}, \mathrm{C}_{3} \mathrm{H}_{8}$ and temperature between 2005 and 2010 with a static photochemical model (Moses and Greathouse 2005) and find substantial effects of circulation and transport in addition to variations due to chemistry. Seasonal change of temperature, composition and aerosol is extensively reviewed by Fletcher et al. (2018c).

\subsection{Zonal Jets and Temperatures}

Winds are motions of the atmosphere relative to the rotating planet, and for Jupiter, Uranus and Neptune the wobble of the planet's tilted magnetic field provides the planetary rotation rate. Saturn's field is tilted less than $0.007^{\circ}$ and therefore does not provide a physicallybased reference frame (Cao et al. 2020). For locating cloud features, scientists use a period (10:39:24 s) that was defined following the Voyager encounters, knowing that the computed winds might be systematically too fast or too slow by over $100 \mathrm{~ms}^{-1}$.

Tracking small clouds in sequences of images is the principal way of measuring the winds (Fig. 5). ISS provided the first data at the start of the Cassini mission (Garcia-Melendo et al. 2011b; Porco et al. 2005; Vasavada et al. 2006) and a valuable comparison with Voyager 25 years earlier. VIMS can measure the winds at somewhat deeper levels than ISS. CIRS can measure the gradient of the wind with respect to altitude by measuring the gradient of temperature with respect to latitude and applying the thermal wind equation. Except at the equator, the mean zonal winds - the eastward winds averaged with respect to longitude - are remarkably steady. This conclusion follows, in part, by comparing the mean zonal winds during Voyager times with those measured by Cassini 25-30 years later (Antunano et al. 2015; Choi et al. 2009; Li et al. 2011, 2013; Vasavada et al. 2006). Images taken at wavelengths where methane is a strong absorber tend to sample higher altitudes than those where methane 

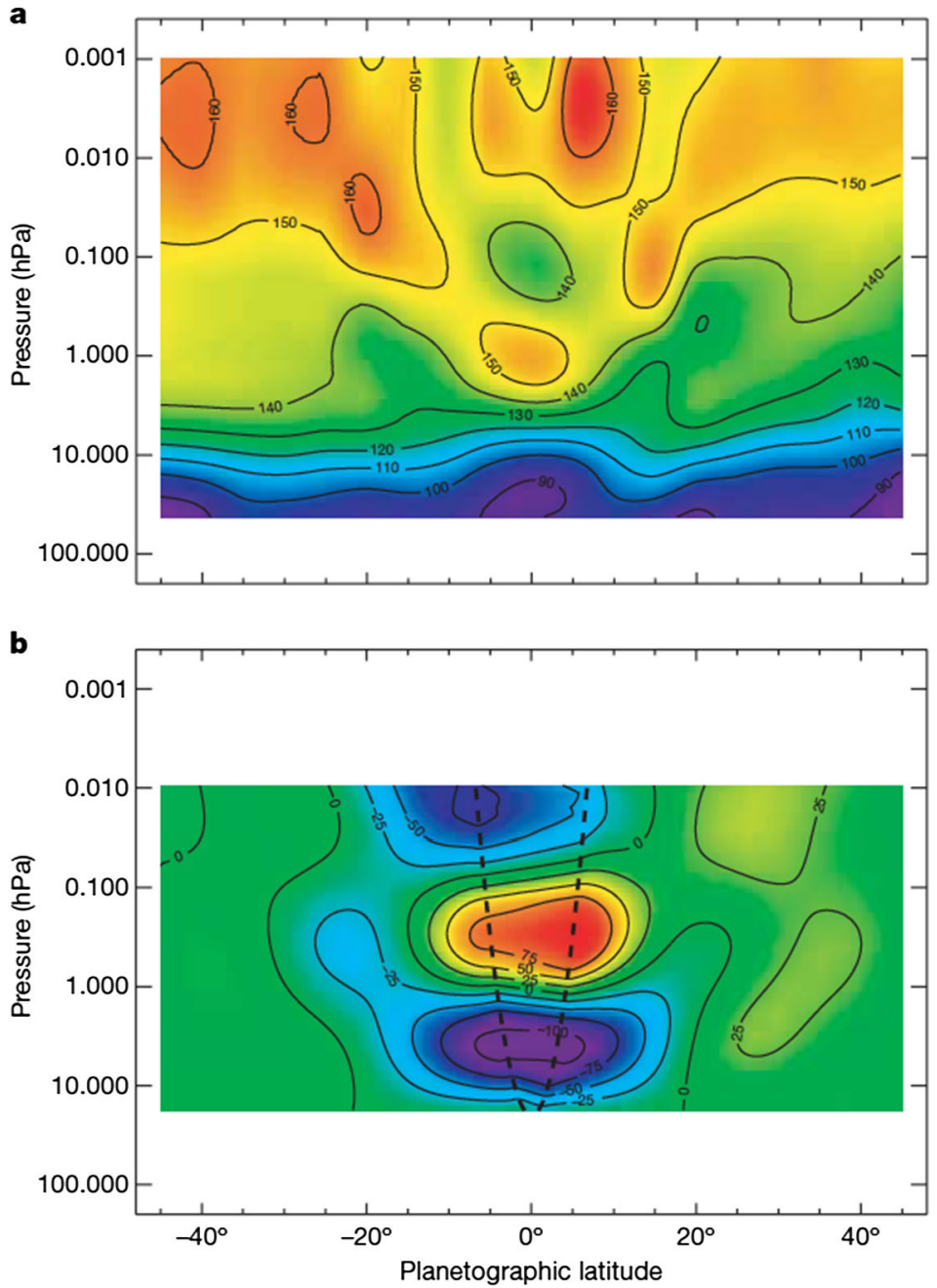

Fig. 6 Oscillation of temperature (upper panel) and mean zonal wind (lower panel) at the equator from Cassini CIRS. The pattern moves down with time and has a $\sim 15$-year period (Orton et al. 2008; Fouchet et al. 2008; Guerlet et al. 2018). A similar pattern on Jupiter has a period of $\sim 4$ years (Orton et al. 1991). The Figure is from Fouchet et al. (2008)

is transparent. Vertical shears show up in the eastward jets (Garcia-Melendo et al. 2009, 2011a, 2011b), which is consistent with the horizontal temperature gradient and the thermal wind equation

At the equator, the speed of the zonal wind in the upper troposphere and stratosphere oscillates with a $\sim 15$-year period, which is half the Saturn year (Fouchet et al. 2008) (Fig. 6). In that sense, it is a semiannual oscillation. Since vertical shear in the zonal wind is related to horizontal gradients of temperature through the thermal wind equation, the oscillation reveals itself in the temperatures on either side of the equator. It shows up in the lower panel of Fig. 4 as the tripolar structure spanning the equator. Similar oscillations occur in the stratospheres of Earth and Jupiter. On Jupiter, the period is about four years. On 
Earth, there are two oscillations: the semi-annual oscillation (SAO), which has a period of six months, and the quasi-biennial oscillation (QBO), which has a period of about 22 months. The QBO is thought to be forced by waves propagating up from the troposphere, with feedback between the wind profile and the altitude where the waves deposit their zonal momentum. Downward propagation of the wind profile and the upward-propagating waves needed to support it have been extracted from CIRS limb observations (Guerlet et al. 2011, 2018).

The SAO period is the time between successive solstices, regardless of sign, and it could be forced by radiation. Sinclair et al. (2014) compare temperatures and gaseous abundances from Cassini with those from Voyager exactly one Saturn year later. They find changes, but most of them could be accounted for by a change in phase of the stratospheric oscillation, suggesting that the oscillation is only "quasi-semiannual". For a review, see Read (2018).

In preparation for the Cassini encounter, Voyager and Hubble Space Telescope observations were used to document the variability of the zonal wind in the equatorial stratosphere (Perez-Hoyos and Sanchez-Lavega 2006; Sanchez-Lavega et al. 2000). Sanchez-Lavega et al. (2007) and Garcia-Melendo et al. (2010) used Cassini ISS images in the methane bands to further document the vertical wind shear and its temporal variability. Li et al. (2008) used Cassini CIRS data, where the oscillation is revealed in stratospheric temperatures, and Li et al. (2011) showed that the temperatures and cloud-tracked winds are consistent with the QBO oscillation mechanism. Schinder et al. (2011) used radio occultations by the Cassini spacecraft to measure temperature and showed that the zonal wind profile propagates downward as on Earth, consistent with the wave forcing mechanism of the QBO. Downward propagation also shows up in the thermal infrared maps based on CIRS data (Fouchet et al. 2008; Guerlet et al. 2011). Sanchez-Lavega et al. (2016) used ISS data to track individual features in the upper haze and clouds and further demonstrated the intense vertical shears and temporal variability.

A fundamental question dating back to Voyager days (Gierasch et al. 1986), for both Jupiter and Saturn, is why the zonal jets and the flow around the large ovals decay with height above the tops of the clouds. For Saturn this decay is seen, first, by tracking clouds in filters that sample different altitudes, like the winds within $\pm 15^{\circ}$ of the equator in Fig. 5 (Garcia-Melendo et al. 2009, 2011b). Second, the decay is inferred from the temperature gradients with respect to latitude-the spiky excursions in Fig. 3 and the little bumps and wiggles at mid latitudes in Fig. 4. These bumps and wiggles are not noise; the peak gradients are located at the latitudes of the zonal jets, and the thermal wind equation integrated upward from the cloud tops indicates that both the eastward jets and the westward jets are losing speed with altitude (Fletcher et al. 2008; Read et al. 2009b). The opposite behavior seems to occur from 2 bars to 300-500 mbar, as seen in Cassini VIMS images (Studwell et al. 2018). The temperature gradients could be forced by differential heating, or the decay of the jets could be forced by wave transport of momentum or both, since the two are linked by the thermal wind equation. Either way, the forcing has not been properly identified and the question remains open.

\section{The Great Storm of 2010-2011}

\subsection{Storm Clouds and Convection}

Saturn is prone to large-scale eruptions (Fig. 7). They start with a small cloud that grows rapidly during its first week and expands zonally, until after 2 or 3 months the disturbance 
Fig. 7 Saturn imaged by Cassini ISS on February 25, 2011. The great storm of 2010-2011 is clearly visible in a band centered at $30-40^{\circ}$ latitude. Since its appearance on December 5, 2010, the head of the storm had drifted west and overtaken the tail (PIA 12826)
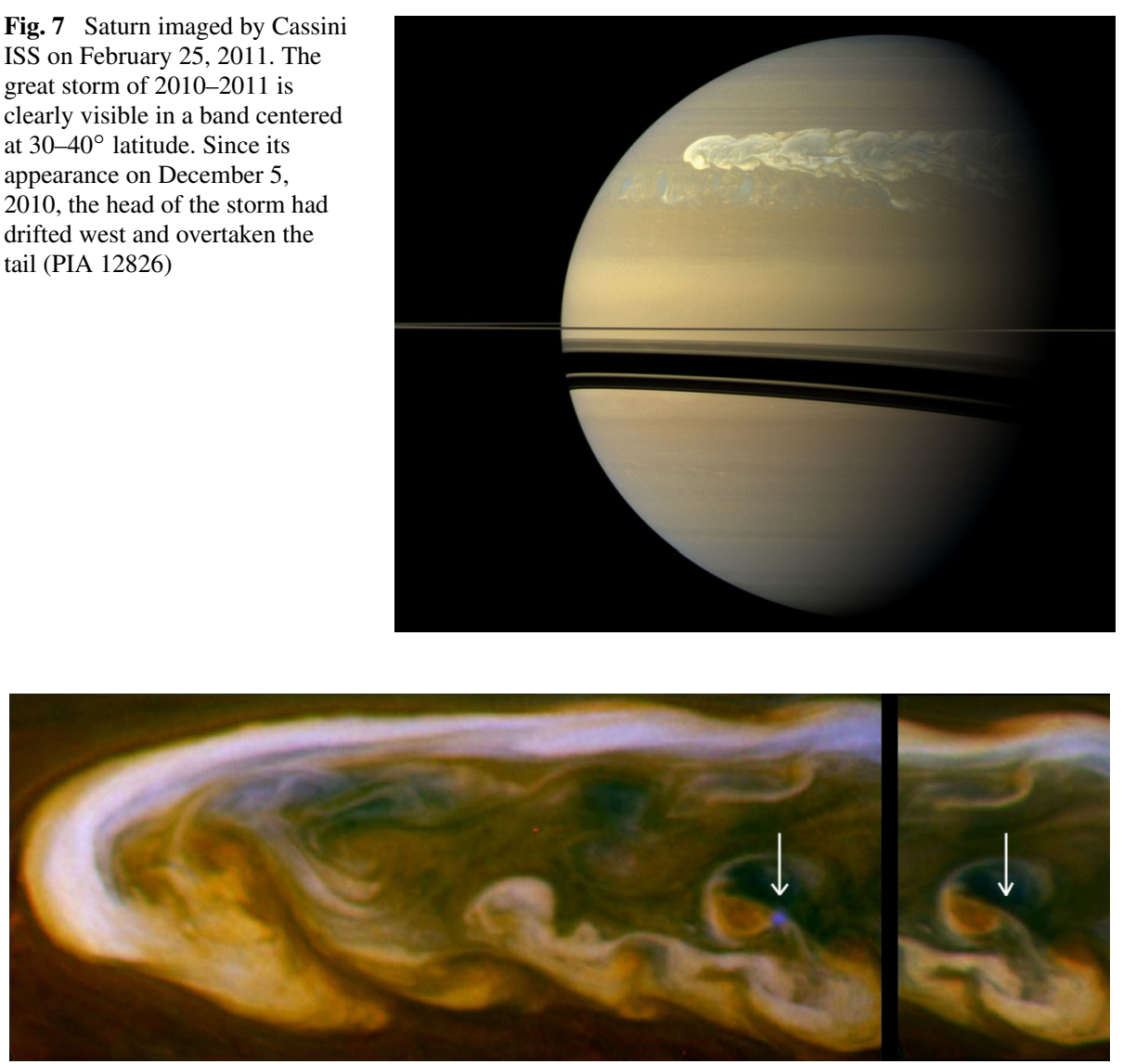

Fig. 8 Lightning flash in Saturn's great storm of 2010-2011. The color composite consists of three images taken in rapid succession at three different wavelengths. A lightning flash occurred while the blue-filtered image was taken, making a blue spot in the composite image, left. The same region was imaged 30 minutes later and did not see a lightning flash, right (PIA14921)

has fully encircled the planet within its latitude band. The paper by Sanchez-Lavega (1994) is the definitive pre-Cassini review of these phenomena. He calls them Great White Spots, and he lists five of them before the Cassini era: 1876, 1903, 1933, 1960, and 1990. This gives an average periodicity of 28.5 Earth years, which is close to Saturn's year of 29.46 Earth years. The corresponding latitude bands of these storms are $8 \pm 3^{\circ}, 36 \pm 2^{\circ}, 2 \pm 3^{\circ}, 58 \pm 1^{\circ}$, and $12 \pm 1^{\circ}$, all in the northern hemisphere. Whether the disturbances are in a unique class or just the largest in a continuous distribution of sizes is uncertain. Intermediate-scale storms do exist (Sanchez-Lavega et al. 2020a). And whether the disturbances are locked in phase to Saturn's year is also uncertain, because within each latitude band the period is much longer than a Saturn year. And the great storm of 2010-2011 came 10 years too early to satisfy the annual cycle hypothesis.

Saturn's great storm of 2010-2011 was a singular event in Cassini's 13-year tour of the Saturn system (Sanchez-Lavega et al. 2011, 2018). It began as a small $(\sim 1000 \mathrm{~km})$ spot captured in a routine ISS image on December 5, 2010. Cassini Radio and Plasma Wave Spectrometer (RPWS) had been detecting lighting discharges (Fig. 8) from the storm for 


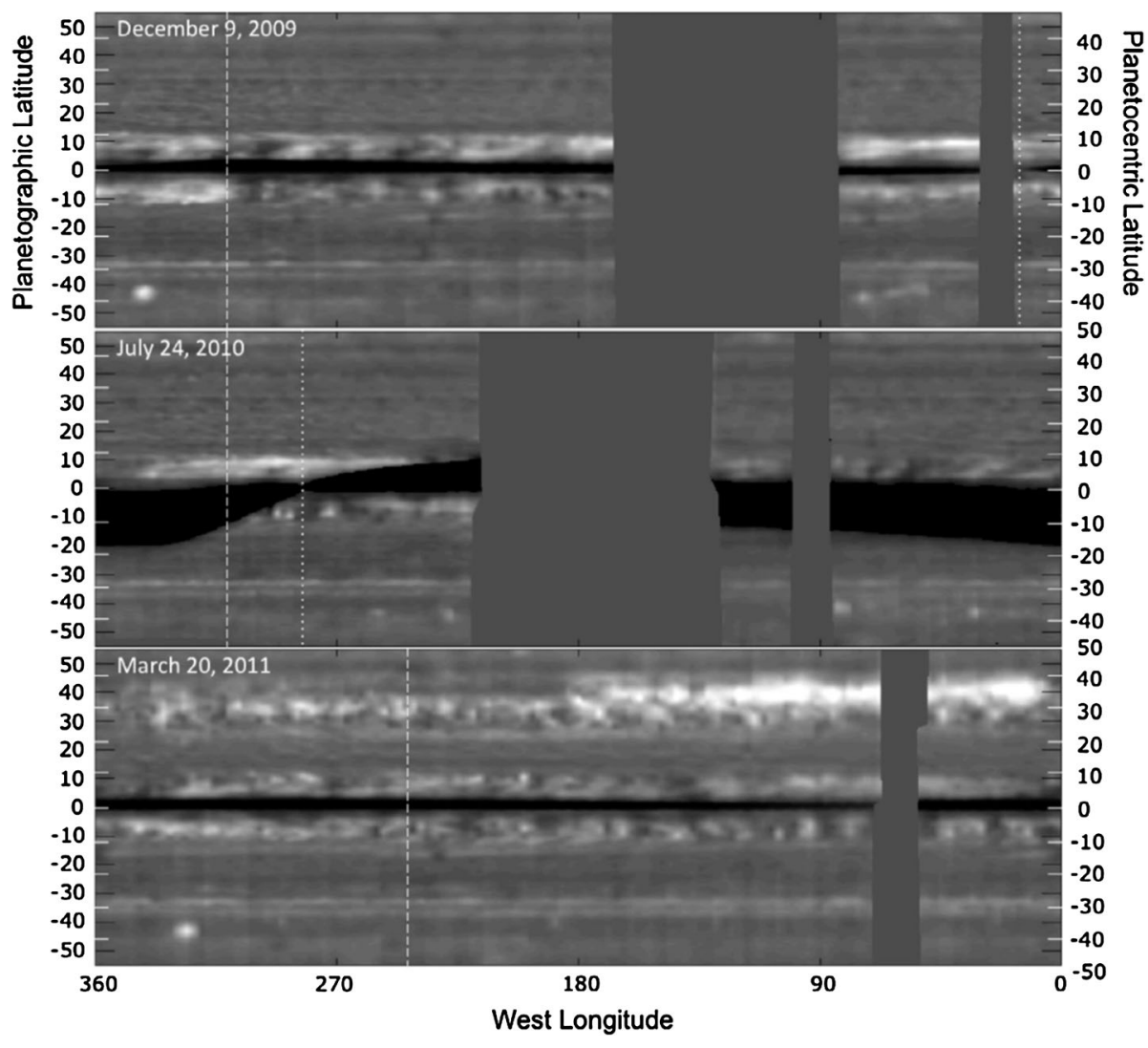

Fig. 9 Cylindrical projection of Saturn's thermal emission at $2.2 \mathrm{~cm}$ wavelength, obtained by the Cassini RADAR in passive (non-transmitting) mode. The dark band at the equator is due to the rings, which are colder than the planet itself. The top two panels show the planet before the great storm. The lower panel shows warm emission at the location of the storm. Ammonia is the principal absorber at $2.2 \mathrm{~cm}$ wavelength, so the warm emission is due to ammonia depletion allowing radiation from deeper levels to reach the detector (Janssen et al. 2013)

a few hours when the image was taken (Fischer et al. 2011). The lightning continued for the $\sim 7$ month lifetime of the storm as the westward-moving head left behind a tail that eventually wrapped around the planet. In its mature phase, the storm filled a latitude band $10,000 \mathrm{~km}$ wide that was centered at a planetocentric latitude of $35^{\circ}$. Amateur astronomers with Earth-based telescopes were following the storm within days of its appearance, and Cassini began systematic imaging in January 2011.

Visible-light images of clouds in the troposphere (Sanchez-Lavega et al. 2012; Sayanagi et al. 2013) showed that the head spawned large $(10,000 \mathrm{~km}$ diameter $)$ anticyclones that drifted off to the east and became part of the tail. Amateur astronomers provided nearly continuous coverage. The storm seems to have originated from a feature called the string of pearls, a train of cyclones at the latitude of the great storm (Sayanagi et al. 2014). GarciaMelendo et al. (2013) are able to reproduce many of the observed morphological features of the storm with a prescribed heat source moving at a prescribed velocity. Li and Ingersoll (2015) present a theory that reproduces the observed drying (low mixing rate of ammonia) of the storm's latitude band (Fig. 9) (Janssen et al. 2013; Laraia et al. 2013) as well as 


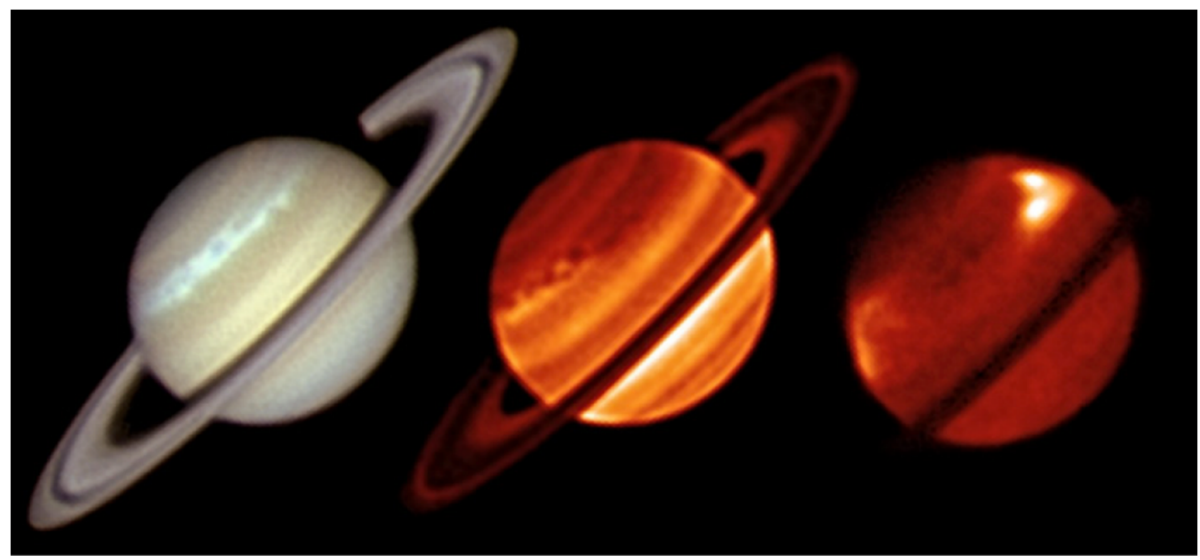

Fig. 10 Visible light image, left, and infrared images sensitive to temperatures in the 200-500 mbar level, center, and the 1-10 mbar level, right, on January 19, 2011. The stratospheric beacon stands out in the right image. The visible light image was from the International Outer Planet Watch database (Hueso et al. 2010), and the infrared images were from the Very Large Telescope (VLT) in Chile (Fletcher et al. 2011b)

the multi-decadal interval between great storms. The latter is tied to mass loading, i.e., the stabilizing effect of water and ammonia condensation in a low molecular weight $\left(\mathrm{H}_{2}\right.$ and $\left.\mathrm{He}\right)$ atmosphere. Above a critical abundance of the condensates, the atmosphere becomes stable after a convective event, and the time needed to destabilize the atmosphere is decades. Based on the $\mathrm{C} / \mathrm{H}$ ratios for Jupiter and Saturn, assuming the same enrichment factors relative to solar for $\mathrm{O} / \mathrm{H}$, Saturn is above the critical value and Jupiter is below it, which could explain why only Saturn has these great storms.

\subsection{Chemical Tracers and Vertical Motion}

Thermal infrared spectroscopy by Cassini CIRS revealed effects of the storm that penetrated into the stratosphere to the $1 \mathrm{mbar}$ level (Fletcher et al. 2011b). Subsidence of air in the stably-stratified stratosphere produced beacons as much as $16 \mathrm{~K}$ warmer than their surroundings (Fig. 10). By May 5, 2011, the beacons had reached temperatures of $226 \mathrm{~K}$, about $80 \mathrm{~K}$ warmer than the surrounding stratosphere (Fletcher et al. 2012; Fouchet et al. 2016). The storm produced a 100-fold increase of ethylene in the stratosphere near the beacon (Hesman et al. 2012). Photochemical models cannot explain this increase, so some dynamical mechanism must be at work (Cavalie et al. 2015; Moses et al. 2015). Temperatures in the troposphere at the latitude of the storm increased by $3 \mathrm{~K}$, and the para fraction of $\mathrm{H}_{2}$ decreased by about 0.4 , indicating warm air upwelling from below (Fig. 11) (Achterberg et al. 2014). The speed of the upwelling is uncertain partly because the time constant for approach of para $\mathrm{H}_{2}$ to equilibrium is uncertain and partly because the existence of a hydrogen dimer complicates the interpretation of the spectrum (Fletcher et al. 2018a).

The 2010-2011 storm seems to have affected clouds and haze, both at the latitude of the storm and farther away. Sromovsky et al. (2013) used VIMS spectra to study the composition of the cloud particles lofted by the storm, and found evidence of ammonia ice, water ice, and ammonium hydrosulfide. This is the first spectroscopic evidence of water ice in Saturn's atmosphere and indicates upwelling from $200 \mathrm{~km}$ below the tops of the ammonia clouds. Further study of the aftermath of the storm reveals clearing of the ammonia cloud with 


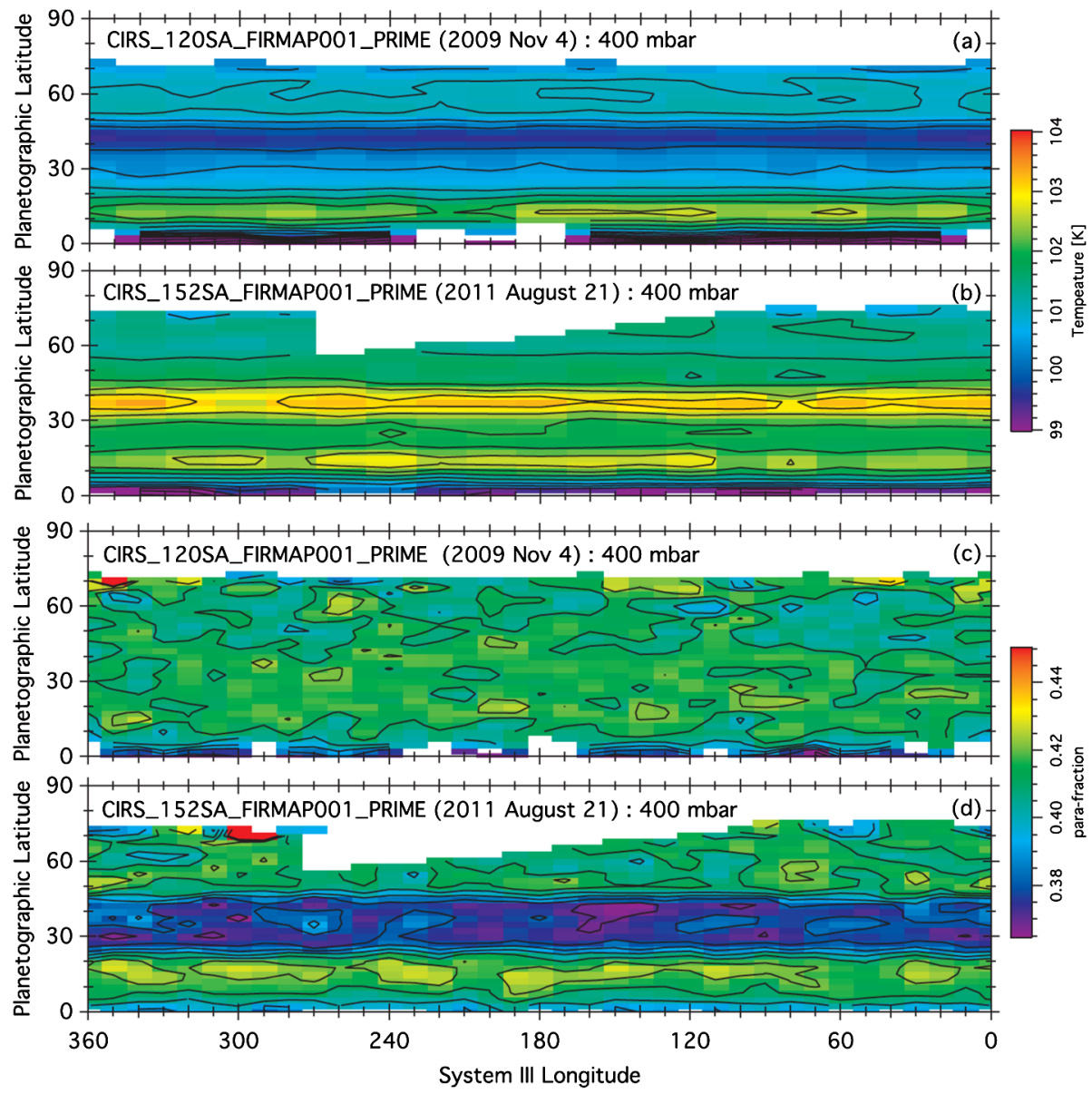

Fig. 11 Cassini CIRS cylindrical projections showing evidence of air being dredged up from below. The top two panels show tropospheric temperature before and after the storm and the band from $30-40^{\circ}$ planetographic has warmed by several degrees. The bottom two panels show the two chemical states (nuclear spins parallel and nuclear spins anti-parallel) of molecular hydrogen $\mathrm{H}_{2}$ before and after the great storm. The $30-40^{\circ}$ band shows a low para fraction, which indicates that the air has risen from below cloud base (Achterberg et al. 2014)

some residual particles of other species (Sromovsky et al. 2016). According to an analysis of VIMS data, a residual haze layer persisted in the upper troposphere and lower stratosphere as well (Oliva et al. 2016). The great storm seems to have caused a $10 \mathrm{~K}$ warming of the middle atmosphere $(0.5-5$ mbar $)$ at the equator, providing evidence of teleconnections between latitudes (Fletcher et al. 2017).

\section{Vortices, Mean Flow and Eddies}

\subsection{North Polar Hexagon}

Saturn's hexagon is a six-lobed meandering pattern in an eastward jet centered at $75^{\circ}$ north latitude (Fig. 12). The excursions in latitude are approximately $\pm 1^{\circ}$, which gives the struc- 


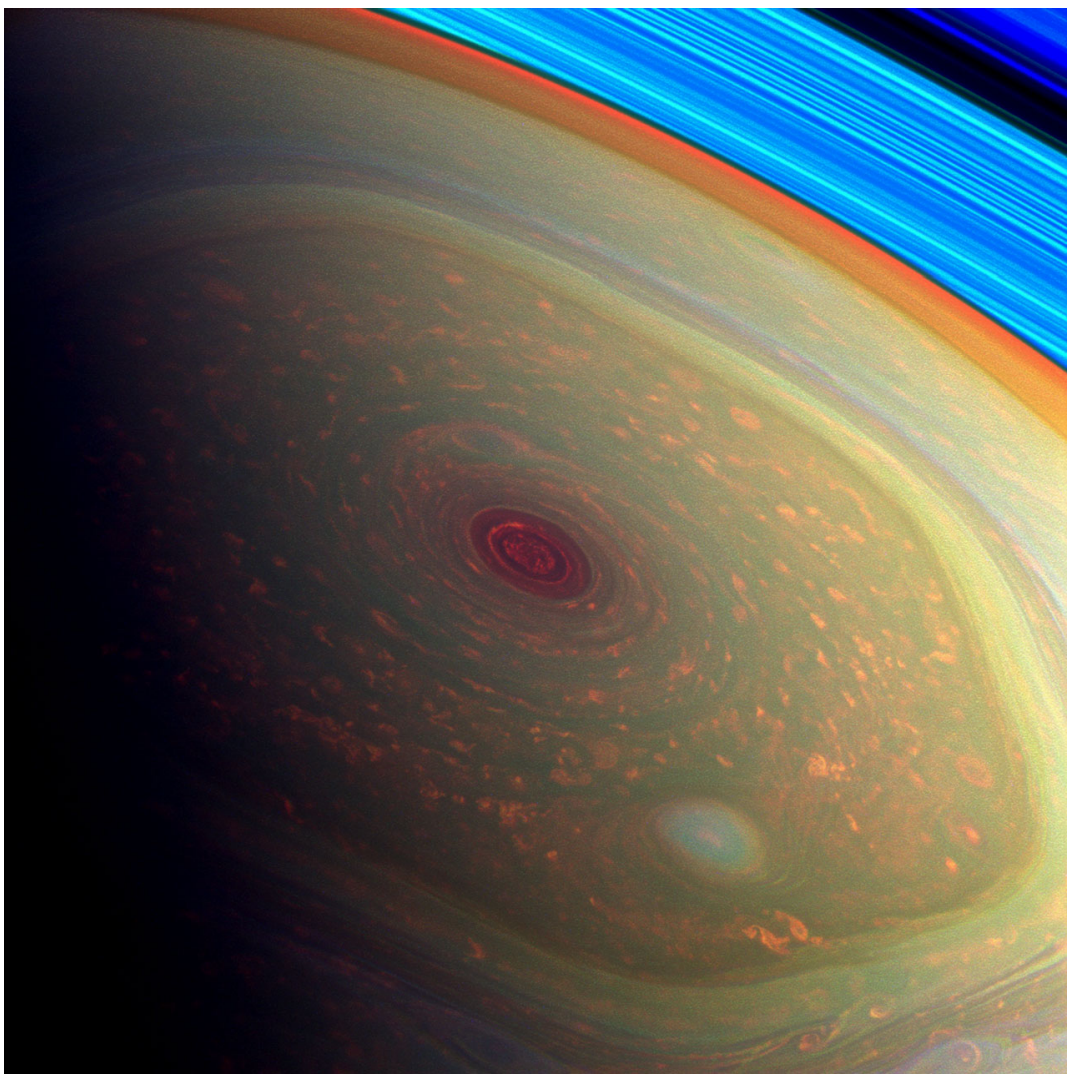

Fig. 12 Saturn's north polar hexagon and polar cyclone. This false color image was taken on November 27, 2012 by the Cassini ISS. The MT3 and MT2 images, which look dark due to absorption by methane gas in the atmosphere, are projected as blue and green, respectively. The CB2 image, which is not sensitive to methane absorption, is projected as red. The color balance is chosen to make the planet's atmosphere look realistic. The rings look bright blue because there is no methane gas between the rings and the spacecraft. The red spot in the center extends from the pole to a latitude of $88-89^{\circ}$. It looks red in the false-color image because the clouds are deep and methane gas absorbs the sunlight before it can reflect off the clouds and reach the spacecraft. The spot is a cyclone with winds of $\sim 100 \mathrm{~ms}^{-1}$ [PIA14946]

ture its polygonal shape. Larger excursions would give it a more sinusoidal, wave-like shape. The hexagon

was discovered in Voyager images taken in 1980 and was discovered again by Cassini VIMS, before the spring equinox when the North Pole was still in darkness (Baines et al. 2009b). Its effects extend into the stratosphere to altitudes of 0.5 mbar (Adriani et al. 2015; Fletcher et al. 2018b; Pryor et al. 2019; Sanz-Requena et al. 2018; Sanchez-Lavega et al. 2020a, 2020b). Based on ISS and VIMS imaging, the hexagon is a meandering jet whose 6-lobed pattern moves slowly or not at all relative to the nominal rotation rate for Saturn, which was defined following the Voyager encounter (Desch and Kaiser 1981). Small clouds within the jet move at $\sim 125 \mathrm{~ms}^{-1}$ relative to the pattern. Thus the hexagon is like a road fixed in the Voyager reference frame, and the clouds in the jet are cars moving along the road. Based on the hexagon's long-term stability, Sanchez-Lavega et al. (2014) argue that it is a deep-seated feature that could reveal the true rotation of the planet. 
Each pole of Saturn is occupied by a single isolated cyclonic vortex with peak winds of $150 \mathrm{~ms}^{-1}$ (Dyudina et al. 2008, 2009; Sanchez-Lavega et al. 2006; Antunano et al. 2015; Liu et al. 2019). The latitude of the peak winds is $88.5^{\circ}$ (Antunano et al. 2015), which means that the average relative vorticity within $1.5^{\circ}$ of the poles is equal to 0.57 times the local planetary vorticity. Based on Cassini ISS, VIMS, and CIRS data, Dyudina et al. (2008) and Dyudina et al. (2009) interpreted the vortex as a hurricane-like eye with eyewall clouds at $88.5^{\circ}$ extending $20-70 \mathrm{~km}$ above the clouds at the pole. Sromovsky and Fry (2019) argued that an abrupt change of optical thickness could mimic the apparent eyewalls. The region has a 4-7 K warm core extending from the upper troposphere into the stratosphere (Achterberg et al. 2018). The warm core and stable stratification imply downwelling, which is consistent with the observed low phosphine abundance in the core (Fletcher et al. 2008). Downwelling is also consistent with the near absence of high- and medium-level clouds in the eye (Sromovsky et al. 2020), although isolated convective clouds are present (Baines et al. 2018). Outside the eyewall are numerous anticyclonic vortices suggesting a convective origin (Dyudina et al. 2009). Sayanagi et al. (2017) compared the north and south polar cyclones and attributed the differences to seasonal effects. Polar phenomena are extensively reviewed by Sayanagi et al. (2018).

\subsection{Eddy Mean-Flow Interaction}

Giant planet atmospheres are a superb laboratory for studying the dynamics of rotating fluids. Cloud tracking provides estimates of the winds, and the lack of continents and oceans provides a simpler setting than on Earth. The Cassini mission advanced this field, not only by providing 13 years of observations of Saturn but also by providing a 3-month flyby of Jupiter with an upgraded suite of instruments compared with those on the Voyager flybys. The Jupiter observations are discussed in Sect. 8 of this paper. Here we focus on observations and models of the jets and vortices in Saturn's atmosphere. Saturn's atmospheric dynamics is extensively reviewed by Showman et al. (2018).

The eddy momentum flux is a fundamental quantity. Eddies are the residuals after the time- and longitude-dependent mean winds $\bar{u}$ and $\bar{v}$ have been subtracted off. The eddy eastward wind is $u^{\prime}$ and the eddy northward wind is $v^{\prime}$. By definition the means of $u^{\prime}$ and $v^{\prime}$ are zero, but the mean of their product $\overline{u^{\prime} v^{\prime}}$ may be non-zero. Multiplied by the gas density, $\overline{u^{\prime} v^{\prime}}$ is the eddy momentum flux - the northward flux of eastward momentum. Using ISS data, Del Genio et al. (2007) and Del Genio and Barbara (2012) measured $\overline{u^{\prime} v^{\prime}}$ as a function of latitude (Fig. 13). The middle panel of Fig. 13 shows negative values of $\overline{u^{\prime} v^{\prime}}$ at $8-18^{\circ}$ north latitude, indicating southward transport of eastward momentum by the eddies. The positive values at $8-18^{\circ}$ south latitude indicate northward transport of eastward momentum. In other words, the eddies are subtracting eastward momentum from the westward winds beyond $\pm 8-18^{\circ}$ and adding it to the eastward winds within $8^{\circ}$ of the equator. This seems counterintuitive, but it also occurs on Earth and Jupiter. The basic requirement is that the eddies have a source of energy that is distinct from the kinetic energy of the zonal jets.

If the eddy momentum flux were the only process acting, the jets could not be in equilibrium. To balance the eddies, there has to be an equatorward meridional flow $(\bar{v}<0$ in the north, $\bar{v}>0$ in the south), which carries low-angular momentum air to the equator. Thus air is approaching the equator from both sides. The net result is downwelling at the equator and upwelling where the meridional flows originate, i.e., in the $\pm 8-18^{\circ}$ latitude range. This picture applies in the troposphere and is consistent with the inferences drawn from the distributions of $\mathrm{PH}_{3}$ and $\mathrm{AsH}_{3}$ in Fig. 2.

Higher up, in the upper troposphere and stratosphere, other processes dominate. In the last paragraph of Sect. 3 we discussed the decay of zonal winds with height. Such decay 

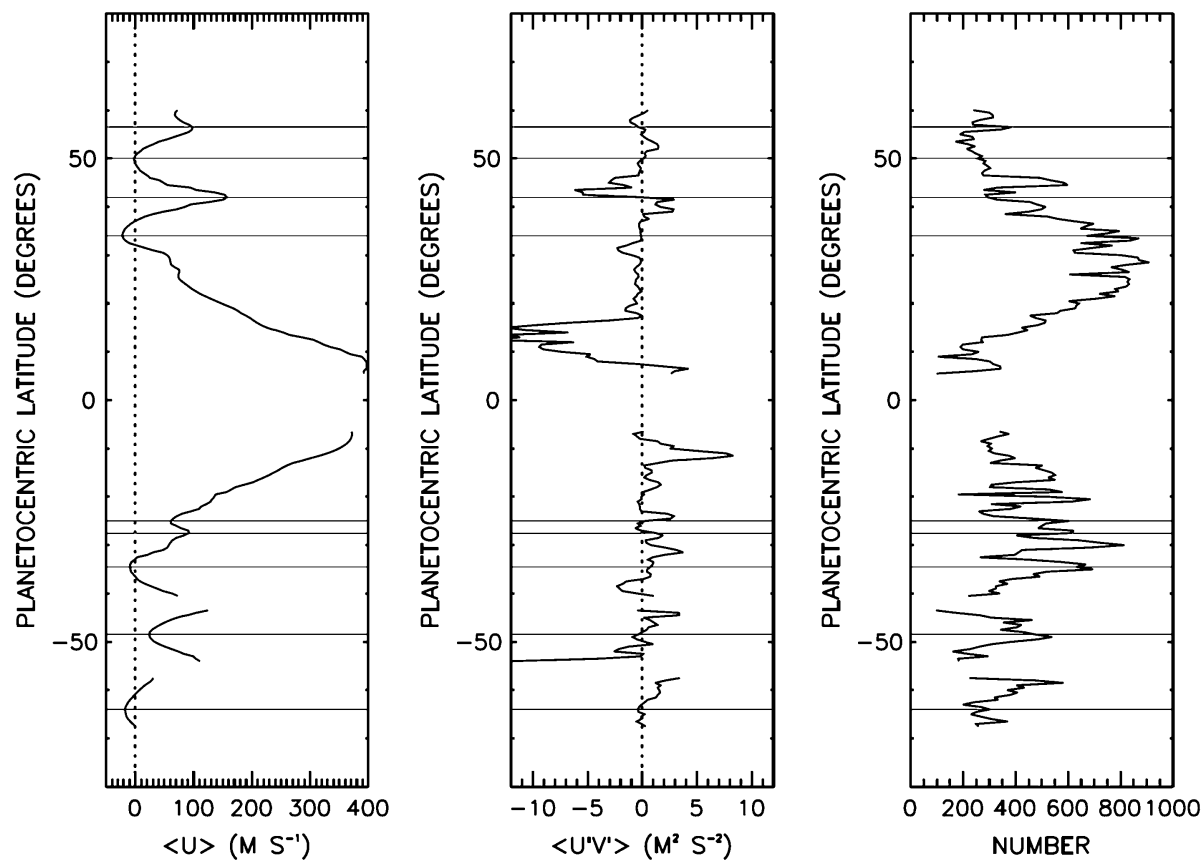

Fig. 13 Eddy momentum transport for Saturn from Cassini ISS. The eastward and northward eddy winds $\mathrm{u}^{\prime}$ and $\mathrm{v}^{\prime}$ are the departures from the zonal means. Their product $\overline{u^{\prime} v^{\prime}}$ averaged over longitude and multiplied by density is the northward eddy flux of eastward momentum. The fact that this quantity has the same sign as $\partial \bar{u} / \partial y$, which is the increase of the mean eastward wind with latitude, says that the eddies are putting energy into the jets and not the reverse. The left panel is $\bar{u}$, the middle panel is $\overline{u^{\prime} v^{\prime}}$, and the right panel is the number of velocity measurements per $0.5^{\circ}$ latitude bin (Del Genio and Barbara 2012)

requires a braking process - a force to the west acting on the eastward winds within $\pm 8^{\circ}$ of the equator (Fig. 5), and a force to the east acting on the westward jets farther from the equator. This force has not been identified, but it acts from the cloud tops up into the stratosphere. To balance it, there must be meridional flow, but that meridional flow must be diverging at the equator and converging between the eastward jet at the equator and the westward jets on either side of the equator. This leads to upwelling at the equator and downwelling on either side of the equator. This picture is qualitatively consistent with the inferences drawn from the $\mathrm{NH}_{3}$ distribution shown in Fig. 2 but is opposite to those drawn from $\mathrm{PH}_{3}$ and $\mathrm{AsH}_{3}$. Although the braking force has not been identified, it is based on the decay of zonal jets with height and is consistent with the stacked, oppositely-directed Hadley cells suggested by the tracer distributions. Zuchowski et al. (2009) show that a simple analytic model forced by Newtonian relaxation of heat and momentum, properly aligned, could give rise to the two-cell system.

Zonal jet stability is another important observation. Stability depends on the potential vorticity gradient (Antunano et al. 2019), which depends on vertical structure, both of temperature and wind, but a simple but relevant criterion is that the flow is stable if the curvature of the zonal wind profile with respect to latitude does not exceed $\beta$, which is twice the planet's angular velocity times the cosine of latitude divided by the planetary radius. Interestingly, this stability criterion is violated on Saturn near the peaks of the westward jets, especially at high latitudes, indicating that the zonal jets could be unstable (Antunano et al. 2015; 
Read et al. 2009a). That does not mean the flow is unstable, however, as there are other stability criteria that involve vertical structure (Dowling 1995). Liao et al. (2007) argue that a statistical equilibrium occurs as the growing wave saturates. In their global vortex analysis, Trammell et al. (2014) find a correlation between the number of vortices and the westward jet peaks, implying at least some degree of instability at those latitudes.

Waves and vortices are another important feature of giant planet atmospheres. Saturn currently has nothing like the Great Red Spot, which has endured for over 100 years, but it has similar structures. Generally, anticyclones last longer than cyclones, but del RioGaztelurrutia et al. $(2010,2018)$ document a cyclone that lasted for four years. For a comprehensive review of spots and vortices during the Cassini mission, see Hueso et al. (2020). Convective storms can generate waves that transport westward momentum away from their source regions, helping to accelerate eastward jets at the latitudes of the convection (Gunnarson et al. 2018; Liu and Schneider 2015; Sayanagi and Showman 2007). This acceleration must be balanced somewhere, and (Schneider and Liu 2009) argue that the balance occurs through magneto-hydrodynamic drag at 0.3 to $1.4 \mathrm{Mbar}$ where the atmosphere becomes electrically conducting (Liu et al. 2014; Liu and Schneider 2015).

\subsection{Modeling Studies}

Saturn's north polar hexagon at $75^{\circ}$ latitude has inspired several modeling studies, both in the laboratory (Aguiar et al. 2010) and on the computer (Morales-Juberias et al. 2011, 2015; Rostami et al. 2017). Morales-Juberias et al. (2011, 2015) use the EPIC general circulation model with 20 vertical layers, and Rostami et al. (2017) use a shallow water model, which has only one layer. Both models start with an unstable zonal jet at the latitude of the hexagon, and with the right parameter settings the growing wave saturates as a hexagonal pattern that resembles the observed one. Morales-Juberias et al. (2015) get the best fit when the strength of the jet decreases with depth, and Rostami et al. (2017) get the best fit when they include a central cyclone within $2^{\circ}$ of the pole. Central cyclones exist at both poles, and the main observable difference between north and south is that the southern jet is at a lower latitude than the northern one, $70.4^{\circ} \mathrm{S}$ vs. $75.8^{\circ} \mathrm{N}$ planetocentric (Antunano et al. 2015). The main unknown is the radius of deformation, which is related to the mean stratification within the weather layer-from the tops of the ammonia clouds to the base of the water cloud. Why there is only one hexagon is still uncertain.

Saturn's ribbon at $47^{\circ}$ latitude is a less dramatic relative of the hexagon (Sayanagi et al. 2010), since both seem to represent a steady meandering pattern on an eastward jet stream. The models do not explain why the conditions necessary for a long-lived meandering jet stream exist only at certain latitudes on Saturn or why there are no such jet streams on Jupiter. O'Neill et al. $(2015,2016)$ propose a model of Saturn's polar cyclones in which convection at mid latitudes produces a vortex that is cyclonic at the bottom and anticyclonic at the top. The two halves separate, and the cyclonic vortex drifts to the pole and merges with other cyclonic vortices to make a single polar vortex. Brueshaber et al. (2019) report on one-layer shallow-water simulations of poleward drifting cyclonic vortices that merge to form polar vortices, and also reveal a mechanism that separates the polar dynamical regimes of Jupiter, Saturn, and Uranus/Neptune. On the other hand, Antunano et al. (2018) report no meridional migration in the region north of the hexagon, and suggest that mergers do not contribute to the maintenance of the polar vortex. Sanz-Requena et al. $(2018,2019)$ describe the haze and cloud structure in the north pole and hexagon region.

Saturn's great storm has also inspired modeling studies. One model uses an imposed heat pulse and studies its interaction with the ambient zonal flow (Garcia-Melendo and 
Sanchez-Lavega 2017). Another model studies how precipitation of water, because of its high molecular mass relative to hydrogen, can stabilize the atmosphere and inhibit convection for decades due to the long radiative time constant of Saturn's atmosphere ( $\mathrm{Li}$ and Ingersoll 2015). A third model uses a long-term numerical integration of moist convection in a giant planet atmosphere (Sugiyama et al. 2014). The simulations are conducted using a two-dimensional cloud-resolving model that explicitly represents the convective motions and microphysics of $\mathrm{NH}_{3}, \mathrm{NH}_{4} \mathrm{SH}$, and $\mathrm{H}_{2} \mathrm{O}$. It produces intermittent cumulonimbus activity. The time scale is $\sim 60$ days, although it is proportional to the water abundance and is therefore likely to be greater for Saturn than for Jupiter.

Numerical models of Saturn's jets and vortices fall into two categories. One is the conventional general circulation model (GCM), which uses hydrostatic balance in the vertical and is valid for large horizontal scales and small vertical scales (Friedson and Moses 2012; Garcia-Melendo et al. 2007; Trammell et al. 2016; Spiga et al. 2020). The other is the fluid sphere model in which the vertical and horizontal scales are comparable and the flow takes place in thick spherical shells (Aurnou et al. 2008; Heimpel and Aurnou 2007; Liu et al. 2014). Computational limitations make it difficult to develop a single model that includes both small and large vertical scales, although the effort to do that is being made (Heimpel et al. 2016; Cuff and Heimpel 2018). One model (Yadav and Bloxham 2020) suggests that Saturn's hexagon could extend down to where the magnetic field limits the flow speed by interacting with the electrically conducting fluid. More often, the practitioners divide into separate camps and work with different theoretical tools. The goal of these studies is to account for the observations - the number of belts and zones, their associated wind speeds, the stability and other properties of large vortices, and the direction of winds at the equator-prograde for Jupiter and Saturn and retrograde for Uranus and Neptune. The global distribution of temperature and outgoing longwave radiation with respect to latitude (Fig. 3), as well as the temperature gradients with respect to the zonal jets (Fig. 4) are another observable that the models could simulate. See the discussion at the end of Sect. 3.

\subsection{Lightning}

The Cassini RPWS detects the radio waves from lightning at frequencies starting at $1.3 \mathrm{MHz}$ and ranging up to $40 \mathrm{MHz}$ (Fischer et al. 2006a; Fischer et al. 2006b; Fischer et al. 2007; Fischer et al. 2008). A single flash is called a Saturn Electrostatic Discharge (SED), and it lasts for less than the $35.2 \mathrm{~ms}$ integration time of the RPWS instrument (Fig. 14). Fundamentally, the duration of an SED is unknown (Farrell et al. 2007). An SED is not a whistler; it is a freely-propagating radio wave that follows a straight-line path from the source. Although the RPWS is listening virtually all the time, sometimes it detects nothing for months. SED activity is often from a single storm, with SEDs every few seconds for a 5-hour ON period followed by a 5-hour OFF period, when the storm is hidden behind Saturn. Often the storm itself is visible, both in ISS images (Dyudina et al. 2007) and in the images gathered by amateur astronomers at Earth. Criteria used to identify the storm are that it appeared within a day or two after the onset of SED activity and it was always on the side of the planet facing the spacecraft when the RPWS was detecting signals. Thus the number of lightning storms at any one time is likely to be either 0 or 1 . In contrast, Earth has 2000 lightning storms over the globe at all times, and Jupiter has dozens of storms at all times (Little et al. 1999).

Cassini ISS eventually detected the lightning flashes themselves (Dyudina et al. 2010), even on the day side despite the bright background of clouds in sunlight. The secret was to take many short exposures, since a short exposure reduces the background but not the lightning; it does reduce the probability of capturing a flash, so one needs to take many exposures. The diameter of the illuminated spot is about $200 \mathrm{~km}$, indicating that the lightning 
Fig. 14 Saturn electrostatic discharge counts, SEDs, which are roughly equivalent to lightning strikes, over a 2-year period starting in 2004. The SEDs are detected by the RPWS instrument, which is $\mathrm{ON}$ continuously. The lower panel shows ISS coverage of latitudes $-34^{\circ}$ to $-36^{\circ}$, where all the storms were occurring, and it is clear that storms were seen only when the RPWS was detecting SED's (Dyudina et al. 2007)

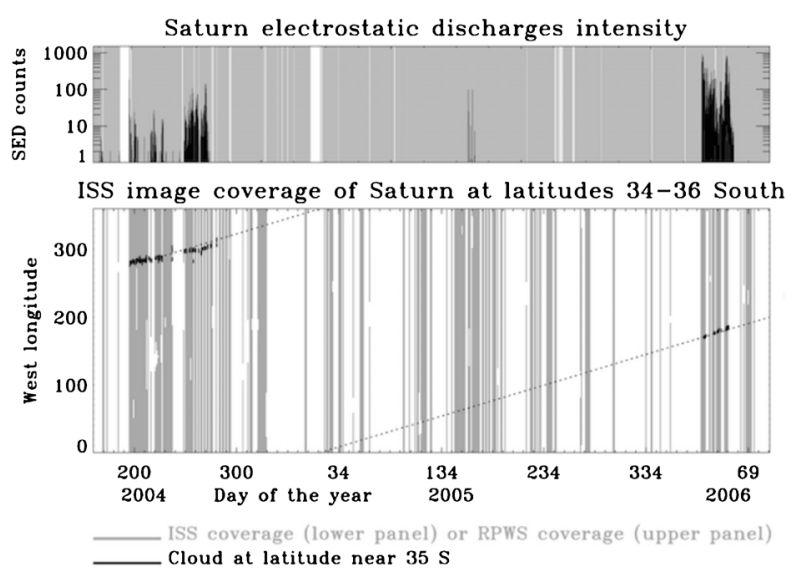

is $125-250 \mathrm{~km}$ below cloud tops. This depth is above the liquid $\mathrm{H}_{2} \mathrm{O}-\mathrm{NH}_{3}$ cloud and may be either in the $\mathrm{NH}_{4} \mathrm{SH}$ cloud or the $\mathrm{H}_{2} \mathrm{O}$ ice cloud.

The great northern storm of 2010-2011 was a copious lightning emitter (Dyudina et al. 2013; Fischer et al. 2011). The RPWS recorded flashes every $0.2 \mathrm{~s}$. The optical energy per flash was about equal to that of the radio energy (Dyudina et al. 2013), although there is considerable uncertainty in both. The optical energy in single flashes ranged up to $8 \times 10^{9} \mathrm{~J}$. The flash rate and total power were hundreds of times greater than those of the smaller southern storms that had been appearing intermittently since the start of Cassini observations. Fischer et al. (2014) proposed that the northern storm could account for a change in the Saturn kilometric radiation (SKR) frequency through its effect on thermospheric winds.

The Saturn lightning results stimulated two reviews of planetary lightning (Yair et al. 2008; Yair 2012). Lightning is an agent of chemical change. Based on VIMS observations, Baines et al. (2009a) proposed that the dark and spectrally featureless clouds associated with giant planet thunderstorms represent small particles of elemental carbon. The particles are postulated to arise from atmospheric methane exposed to high temperatures during the lightning discharge, but Sromovsky et al. (2018) argue that the dark features are more likely regions of reduced optical depth. Dubrovin et al. (2014) study the effect of lightning on the lower ionosphere of Saturn. They find that $\mathrm{H}_{3}{ }^{+}$ions are rapidly produced from the parent $\mathrm{H}_{2}{ }^{+}$ion. On the other hand (Hurley et al. 2012a) searched for but found no correlation between acetylene and thunderstorm activity on Saturn. The Saturn results also stimulated lightning searches throughout the solar system and on exoplanets from Earth-based radio telescopes and from spacecraft (Hodosan et al. 2016; Zarka et al. 2008).

\section{Interior Structure and Rotation}

\subsection{Magnetic and Atmospheric Periodicities}

The periodic variation of the dynamo field has been used to estimate the interior rotation rates of Jupiter, Uranus, and Neptune. However, Saturn's dynamo field axis is so closely aligned with its rotation axis that it has no measurable variation. Taking the misalignment of the other three giant planets, $10^{\circ}$ for Jupiter, $60^{\circ}$ for Uranus, and $47^{\circ}$ for Neptune, the $0.007^{\circ}$ upper bound for Saturn is highly improbable $\left(1\right.$ chance in $10^{6}$ or $10^{7}$ ), given that the probability of the magnetic axis aligning with the rotation axis goes as the square of the angle between them (Dougherty 2017; Cao et al. 2020). The close alignment means that 
there is no detectable wobble in the field as the planet rotates and therefore no periodic signal with which to estimate the rotation period of the planet's interior. The SKR radio emissions are tied to currents in the magnetosphere and ionosphere, and the period is variable from year to year (Fischer et al. 2014), which rules out a direct tie to the interior of Saturn.

There have been various attempts to use the atmospheric periods to estimate the interior rotation rate. They use the data in different ways, but they all derive a period that is near the midpoint of the atmospheric periods. Smith et al. (1982) use cloud tracking and choose a reference frame that minimizes the variance of the cloud-tracked zonal wind with respect to latitude, with 10:31:30 $\pm 30 \mathrm{~s}$ as the result. Anderson and Schubert (2007) chose the reference frame that minimizes the measured shape of the 100 mbar surface from an equipotential, with 10:32:35 $\pm 13 \mathrm{~s}$ as the result. Read et al. (2009b) use the reference frame in which the atmosphere is marginally stable with respect to Arnol'd's second stability criterion, with 10:34:13 $\pm 20 \mathrm{~s}$ as the result. Their method uses ISS and CIRS wind and temperature data to estimate potential vorticity in the range 2-270 mbar. The problem with these approaches is that they ignore the thermal wind equation, which allows vertical shear in the zonal winds. Thus the speed at depth might not match the average speed at the tops of the clouds where the winds and temperatures are measured. Read et al. (2009b) argue that the stability criterion depends on the speed of the longest Rossby waves, which are deeply rooted, perhaps extending to the base of the water cloud, but still the problem remains. For instance, the midpoint of the wind distribution at Earth's upper troposphere would give a period between 22 hours and 23 hours for the rotation of the planet, because the average wind at the top of the troposphere is to the east.

The gravity field (Jacobson et al. 2006) also has information about the rotation rate of the interior (Hubbard et al. 2009). As with all planets, Saturn's gravitational potential can be expanded into spherical harmonics, the leading term of which is the spherically symmetric potential $-\mathrm{GM} / \mathrm{R}$, followed by the zonal harmonics and the tesseral harmonics. The zonal harmonics are axially symmetric, and each term is proportional to a Legendre polynomial of degree $n$ and a dimensionless amplitude factor $\mathrm{J}_{n}$. The tesseral harmonics are the longitudinally-varying part of the gravitational potential. Saturn is a fluid planet, and if it were in equilibrium only the zonal harmonics with even $n$ would be present. The $\mathrm{J}_{n}$ reflect the response of the planet to its own rotation, so one could use them to infer the interior rotation rate if the interior structure were known. Conversely, one could infer the interior structure, e.g., core mass, metallic hydrogen, degree of helium separation, heavy elements in the molecular envelope, etc. if the rotation rate were known (Helled and Guillot 2013; Helled et al. 2015; Hubbard et al. 2009). Further information about the interior could come from the tidal Love number - the magnitude of the planet's response to tidal forces (Lainey et al. 2017). The tidal observations are astrometric data on the orbits of Saturn's moons spanning more than a century and include a large set of Cassini data. The study indicates significant tidal dissipation inside the planet. In all cases, an independent measure of the rotation rate is important for probing the internal structure.

\subsection{Normal Modes and Gravity}

The tesseral harmonics have an effect on the rings, which are a sensitive seismometer for detecting the non-zonal gravity field (Hedman and Nicholson 2013, 2014). The harmonics are of two types. One type has pattern speeds ranging from 807 to 834 degrees per day, for which the corresponding periods are 10:42 $\mathrm{m}$ to 10:22 m, respectively (Fig. 15). These nearly bracket the periods derived from tracking the clouds at various latitudes in Saturn's atmosphere, and could be due to non-zonal structures rotating with the planet. The large range of periods prevents improving on cloud tracking estimates alone. 


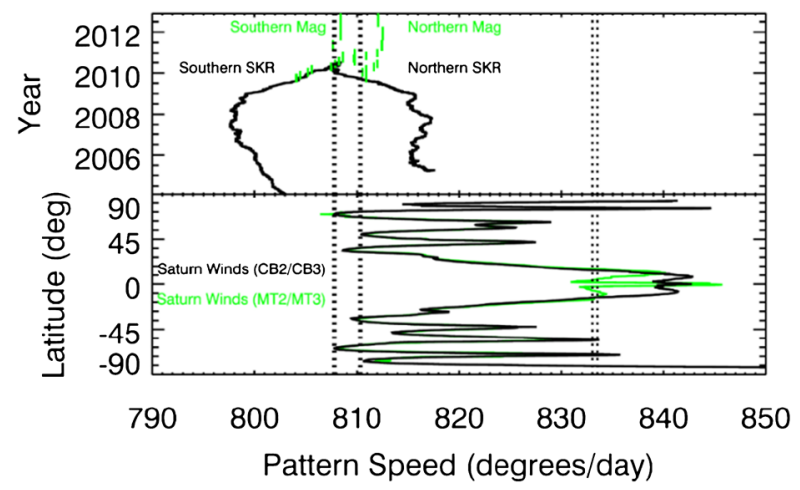

Fig. 15 Ring seismology. The vertical dotted lines are the speeds of non-axisymmetric patterns in Saturn's rings. Only the patterns that match the planet's rotation are shown. They could be due to floating masses in the interior of Saturn. For comparison, periods of exterior magnetic fields, radio emissions, and clouds in the atmosphere are shown. Other patterns with speeds twice as fast are likely due to normal mode oscillations of the planet (Hedman and Nicholson 2014)

The other types of tesseral harmonics have pattern speeds near five hours and are thought to be due to normal mode oscillations inside the planet. The precise frequencies of these normal modes depend both on the interior structure and the rotation rate. Parameters of the interior structure include the mass of the core, the helium mass fraction, the heavy-element mass fraction in the metallic and molecular envelopes, and the pressure at the metallicmolecular transition (Mankovich et al. 2019). For a given rotation rate, the interior parameters are adjusted to give the observed values of $\mathrm{J}_{2}$ and $\mathrm{J}_{4}$. The rotation rate is varied to give the best fit to the observed frequencies of the $\sim 20$ normal modes whose resonant effect on the ring orbits makes them detectable. The planet's rotation affects the frequencies through the Coriolis and centrifugal forces and the ellipticity of level surfaces. The distribution of rotation periods resulting from a broad sample of interior models can be summarized as $10^{\mathrm{h}}$ $33^{\mathrm{m}}$ (Mankovich et al. 2019). The residuals of the fit do not exhibit any strong evidence of differential rotation inside the planet, but differential rotation cannot be ruled out. The rotation period is consistent with predictions by Militzer et al. (2019) who combined interior models, which were constrained by Cassini's gravity measurements, with observations of the planet's oblateness by the Voyager spacecraft. Based on this analysis, a rotation period of 10:33:34 $\pm 55 \mathrm{~s}$ is predicted for the planet's deep interior.

The even zonal harmonics, especially $n=6$ and above, can be used to detect differential rotation inside the planet (Galanti and Kaspi 2017; Kaspi 2013). This method is being used both with Cassini at Saturn and with Juno at Jupiter. The odd harmonics have been used only at Jupiter. The software to analyze these data was developed to be used on both planets (Galanti and Kaspi 2016; Galanti et al. 2017; Kaspi et al. 2016). Cassini produced its best gravity data during six dives between the planet and the innermost ring during the final five months of the mission (Iess et al. 2019). On these dives the spacecraft skimmed 2600$3900 \mathrm{~km}$ above the cloud tops while the radio link to Earth was monitored to determine the gravitational field of the planet and the mass of the rings. The gravity measurements yielded unexpectedly large values of the even zonal harmonics $\mathbf{J}_{6}, \mathbf{J}_{8}$ and $\mathbf{J}_{10}$. These values do not match any interior model that assumes uniform rotation and makes reasonable assumptions about the equation of state and helium rain (Iess et al. 2019). Galanti et al. 2019 confirmed that an acceptable solution can only be found for $\mathrm{J}_{2}$ and $\mathrm{J}_{4}$. 
One can fit all the even harmonics $\mathrm{J}_{2}-\mathrm{J}_{10}$ with a model where unknown winds extend through the planet on cylinders. The best fit is where the atmosphere at the equator rotates 4\% faster than the deep interior, but the models also require a region that rotates more slowly than the deep interior, which is surprising because it is not seen in the cloud tracking data. One can also fit the harmonics by assuming the observed cloud-top winds extend to a finite depth. The best fit is with a finite depth of $9000 \mathrm{~km}$. Qualitatively the two results are the same, since the cloud-top winds are fastest at the equator. The depth of the winds for Saturn is 2-3 times deeper than that for Jupiter, but the difference is explained by the more rapid increase of electrical conductivity with depth on Jupiter. A comparison of the deep dynamics of Jupiter and Saturn in light of Juno and Cassini gravity measurements is presented by Kaspi et al. (2020). Whether magnetic drag suppresses differential rotation directly (Guillot et al. 2018) or indirectly through its effect on the stratification (Christensen et al. 2020) is an open question. Another open question is whether the measured odd zonal harmonics of the gravity field could be due to an extension of the cloud-level zonal winds, as seen on Jupiter by Juno (Kaspi et al. 2018). Kong et al. (2018) and Qin et al. (2020) claim that such an extension does not fit the data, and that the cloud-level winds are confined to a thin layer.

Once the gravity signal of the deep winds is adequately incorporated into models for Saturn's interior, reasonable structures are obtained (Iess et al. 2019; Galanti et al. 2019; Movshovitz et al. 2020). The conditions of helium rain are compatible with predictions from ab initio computer simulations. Cores of heavy elements with 15-18 Earth masses are compatible with the core-accretion hypothesis, which requires an 8-Earth mass core to trigger run-away gas accretion. The enrichment of heavy elements in the planet's molecular envelope of 1-3 times solar is lower than expected but still reasonable. This agreement with other sources of information contrasts with the persistent conundrum for Jupiter, for which the inferred heavy abundances are near or subsolar.

A puzzling feature of this analysis is that the same model of the gravitational field cannot fit all of the six passes in a combined, multi-arc orbital solution (Iess et al. 2019). The residuals represent unmodeled accelerations acting on Cassini over time scales of 20-60 min and could be due to time-varying tesseral harmonics. Convection in Saturn's interior is one possible source. Normal modes varying in amplitude and frequency is another. The data were eventually fit by assuming random accelerations of 10 minute duration acting within \pm 1 hour from pericenter (Iess et al. 2019). The hope is that these high-frequency accelerations do not significantly affect the determination of the even harmonics $J_{2}-J_{10}$.

The amplitudes of the normal modes are a separate issue than the normal mode frequencies. Ordinary turbulent convection as on the Sun (Goldreich and Keeley 1977) falls short by at least six orders of magnitude (Markham and Stevenson 2018) because the kinetic energy is so low. Moist convection apparently has enough thermal energy to drive the modes, should it be available to drive them, but the available kinetic energy falls short by two orders of magnitude (Dederick et al. 2018). Rock storms are an intriguing candidate because their expected energy is greater than for conventional water storms (Markham and Stevenson 2018).

\section{Upper Atmosphere Physics and Chemistry}

\subsection{Chemical Tracers and Ring Rain}

The stratosphere entered in the discussion in the sections entitled composition and chemistry, seasonal variations, and zonal jets and temperatures. To reduce the overlap between those 
sections and this one, we focus here on theoretical models and on the parts of the atmosphere above the stratosphere. The entire subject, upper atmosphere and ionosphere, is reviewed in the book chapter by Nagy et al. (2009). Species like $\mathrm{C}_{2} \mathrm{H}_{2}$ and $\mathrm{C}_{2} \mathrm{H}_{6}$ have chemical lifetimes in the stratosphere that are comparable to a season on Saturn, so they can be used as tracers of the meridional circulation and its annual reversal from north to south. At the equator the zonal winds in the stratosphere exhibit a 15-year oscillation that is accompanied by an oscillation of the temperature distribution symmetric about the equator to maintain thermal wind balance.

The simplest chemical model is a diurnally averaged 1-D photochemical model with eddy diffusion chosen to represent dynamical processes. Moses et al. (2000a) developed a model that couples hydrocarbon and oxygen photochemistry, molecular and eddy diffusion, radiative transfer, and condensation to better constrain the chemical species and to identify the important physical processes that control the abundances. Moses et al. (2000b) consider the role of oxygen by comparing their model results with the observed abundances of $\mathrm{H}_{2} \mathrm{O}$ and $\mathrm{CO}_{2}$. They find that an external source of oxygen is necessary, and they estimate its magnitude. Ring particle diffusion and interplanetary dust are mentioned as likely sources (Moses and Poppe 2017).

During Cassini's final orbits, the spacecraft flew between Saturn's upper atmosphere and its innermost ring, the D ring. The Ion Neutral Mass Spectrometer (INMS) identified $\mathrm{CH}_{4}$, $\mathrm{CO}_{2}, \mathrm{CO}, \mathrm{N}_{2}, \mathrm{H}_{2} \mathrm{O}, \mathrm{NH}_{3}$, and organics in the ring material. The INMS estimate of $>70 \%$ non-icy material exceeded remote sensing estimates and is still an open question (Miller et al. 2020). One source of nanograin particles is Saturn's extended atmosphere extending into the innermost ring (Perry et al. 2018). Another source is electrostatic charging, which could lead to ejection of nanograins out of the ring plane and into the atmosphere by the electromagnetic force (Ip et al. 2016). Further evidence of ring rain is seen in enhanced emissions at latitudes tied to magnetic field lines that cross the equatorial plane at the locations of the inner edges of the A-ring and B-ring and at the orbit of Enceladus (O'Donoghue et al. 2013, 2017).

Spatial and temporal variations in the mixing ratios of chemically reactive species hold clues to the circulation. Figure 16 shows the mixing ratios of $\mathrm{C}_{2} \mathrm{H}_{6}$ and $\mathrm{C}_{2} \mathrm{H}_{2}$ in the stratosphere just before and just after northern spring equinox (blue and red points, respectively). These hydrocarbons are produced as a result of methane photolysis at 0.1 $\mathrm{Pa}$ pressure levels, and they revert to much lower values by photochemical reactions at deeper levels, including $1 \mathrm{hPa}$ where the mixing ratios are shown. Departures from photochemical equilibrium are thought to be due to a meridional circulation. The photochemical loss time of $\mathrm{C}_{2} \mathrm{H}_{2}$ is 3 years and that of $\mathrm{C}_{2} \mathrm{H}_{2}$ is 600 years, and the turnover time of the stratosphere at the $1 \mathrm{hPa}$ level is between the two loss times (Guerlet et al. 2009; Sylvestre et al. 2015).

Hesman et al. (2009) compare the output of the static photochemical model with observations of $\mathrm{C}_{2} \mathrm{H}_{2}$ and $\mathrm{C}_{2} \mathrm{H}_{6}$, and they too find evidence of a meridional circulation. Guerlet et al. (2014) compare the temperatures computed from a radiative equilibrium model with temperatures observed by Cassini CIRS, and they find evidence that other processes, presumably related to dynamics, control Saturn's stratospheric thermal structure. Teanby et al. (2006) place new upper limits on halides in Saturn's stratosphere, but they advise that the abundances in the troposphere could be much larger.

Above the stratosphere, diffusive separation takes over and each gas's density falls off with altitude at its own individual scale height, which depends on its molecular weight. The altitude of the transition is called the homopause. Below the homopause, one finds gaseous hydrocarbons ranging in molecular weight at least up to benzene (Guerlet et al. 2015; Koskinen et al. 2016; Vervack and Moses 2015). These hydrocarbons are 


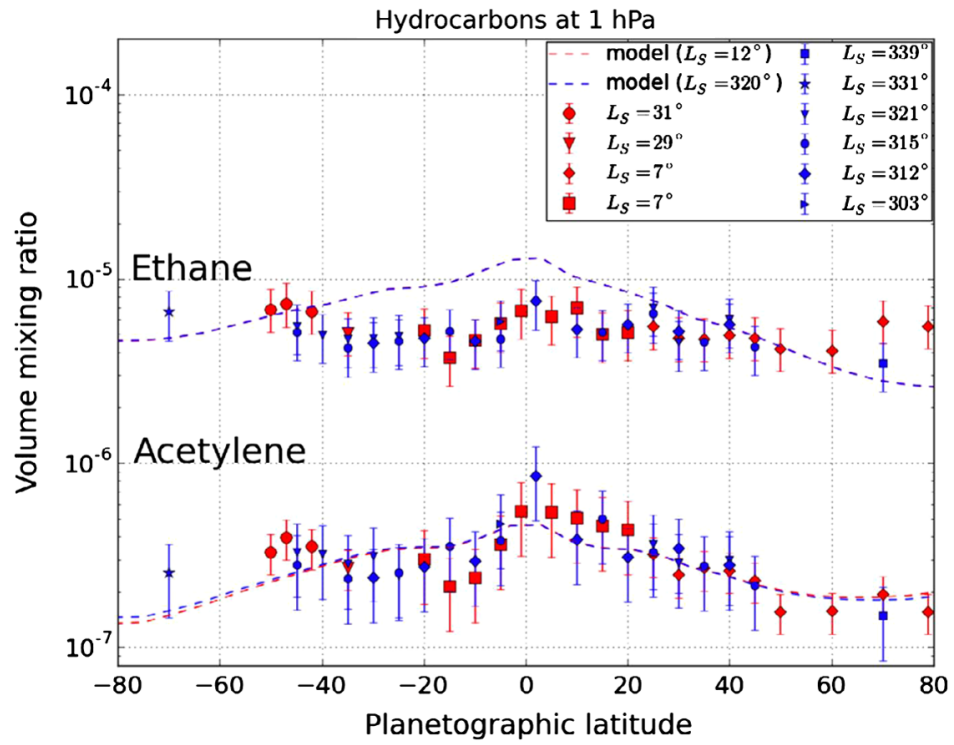

Fig. 16 Hydrocarbons in the stratosphere at 1 mbar pressure. $\mathrm{L}_{s}=0^{\circ}$ and $\mathrm{L}_{s}=360^{\circ}$ are the first day of northern spring on successive years. The dashed lines are the results of a 1D photochemical model with seasons (Moses and Greathouse 2005). Ring shadow is included in the model, which has vertical mixing of species but no meridional circulation. The data are from the CIRS instrument on Cassini (Sylvestre et al. 2015)

the source of stratospheric haze particles at non-auroral latitudes (Moses et al. 2000a; $\mathrm{Kim}$ et al. 2012). Above the homopause the major constituent is $\mathrm{H}_{2}$, which continues into the exosphere where the molecules don't collide with each other except at the exobase (Koskinen et al. 2013). Atomic hydrogen H is also present (Koskinen et al. 2020). Its abundance is generally consistent with models based on photochemistry and transport. The relatively high $(380 \mathrm{~K}$ to $590 \mathrm{~K}$ ) exospheric temperature at low to mid latitudes is a long-standing mystery (Shemansky and Liu 2012; Stallard et al. 2012b). Despite the changing seasons, the high temperatures have persisted from the period of the Voyager flybys through the Cassini era (Koskinen et al. 2015). High temperatures do occur in the auroral zones, but dynamical models strongly suggest that the upper atmosphere is geostrophically balanced and cannot convey the high-temperature air to lower latitudes (Fig. 17). One possibility is that atmospheric gravity waves, which are often overlooked in models, could supply enough torque to overcome the Coriolis force and allow enough meridional overturning to warm the equatorial thermosphere (Mueller-Wodarg et al. 2019; Brown et al. 2020).

The first in situ measurements of the equatorial thermosphere occurred during the final month of the Cassini mission, when the spacecraft skimmed through the upper atmosphere on four periapses and also during the final plunge. The Ion Neutral Mass Spectrometer (INMS) measured the profiles of temperature and composition (Yelle et al. 2018). The best fit to the exospheric temperature is in the range 340 to $370 \mathrm{~K}$, with a value during the final plunge of $354 \mathrm{~K}$. The helium profiles are consistent with diffusive equilibrium, but the methane profiles are not. Even well above the homopause, the $\mathrm{CH}_{4} / \mathrm{H}_{2}$ ratio is nearly constant with height. This points to an external source, the most likely being Saturn's rings (Yelle et al. 2018). 
Fig. 17 Comparison of Cassini UVIS observations with Voyager observations and with a 3-D general circulation model. The model result is represented by the smooth dot-dashed line, and it is significantly low (colder) equatorward of $\pm 60^{\circ}$ in both hemispheres. The problem is that the air from the Polar Regions, heated by auroral currents, cools before it reaches the lower latitudes. What keeps it warm is an ongoing mystery (Koskinen et al. 2015)

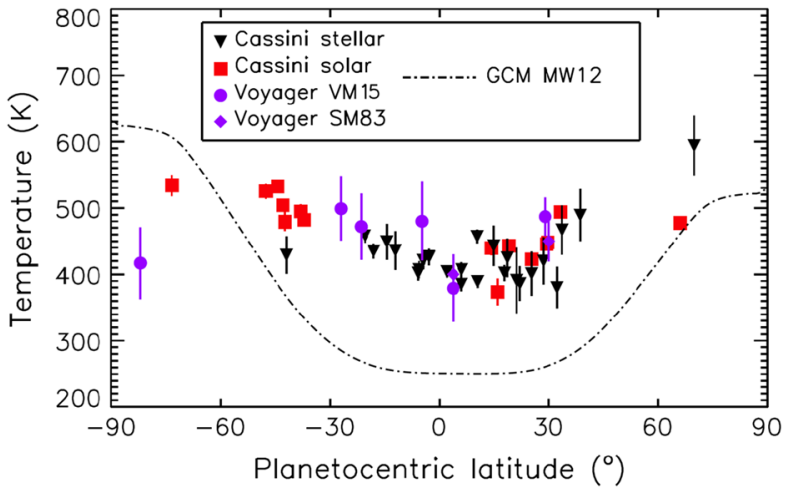

The aurora is caused by precipitating electrons striking the upper atmosphere. The source of the electrons is in the magnetosphere, and the subject is covered mainly in the section entitled Magnetospheres and Plasma Science (MAPS) Discipline Science Results. Some of the questions involve the energy and source of the electrons, how deep they penetrate into the atmosphere, and what effects they have on the temperatures, chemistry, and electromagnetic emissions associated with the aurora.

The aurora is observed in the ultraviolet (Gerard et al. 2013; Gustin et al. 2009, 2010, 2017), visible (Dyudina et al. 2016), infrared (Melin et al. 2011, 2016; O'Donoghue et al. 2014; Stallard et al. 2012a), and radio wavelengths (Lamy et al. 2009, 2013; Ye et al. 2016). The currents associated with the electrons can be observed when the spacecraft flies through an auroral flux tube (Bunce et al. 2014). And although the magnetosphere of Saturn is much less energetic than that of Jupiter, the footprint of the Enceladus flux tube has detectable emissions (Pryor et al. 2011), though not so bright as those of the Jovian moons: The geysers of Enceladus are weaker than the volcanoes of Io, and the magnetic field of Saturn is weaker than the magnetic field of Jupiter.

For a short, broad-brush summary of the magnetosphere, ionosphere, and atmosphere, we recommend Gombosi and Ingersoll (2010). This science objective is captured in detail in the Magnetospheres and Plasma Science (MAPS) Discipline Science Results under the following Cassini science objectives: Conduct in situ studies of Saturn's ionosphere and inner radiation belt, investigate magnetospheric periodicities, their coupling to the ionosphere and how the SKR periods are imposed from close to the planet $\left(3-5 R_{S}\right)$ out to the deep tail, determine the coupling between Saturn's rings and ionosphere, determine the temporal variability of Enceladus' plume (relevant to the Enceladus auroral footprint).

\section{Jupiter Science Results}

\subsection{Winds, Eddies and Long-Lived Ovals}

As it approached and flew past Jupiter in late 2000, Cassini provided three months of valuable data about the atmosphere. It is a photogenic planet; the colorful clouds provide ideal tracers of the winds (Fig. 18). The light and dark bands - the zones and belts-and their associated jet streams have been observed from Earth for more than 100 years. The Great Red Spot (GRS) and its smaller cousins have been observed for just as long. Thus Jupiter is an atmospheric dynamics laboratory, where dynamical phenomena like waves, jets, eddies, and vortices can be studied without the complications of continents, oceans, and large 
Fig. 18 The highest-resolution, full-disk color mosaic of Jupiter ever taken. Jupiter more than filled the field of view of the ISS, so the mosaic was assembled from over 30 individual images, allowing for the planet's rotation as the images were taken [PIA04866]

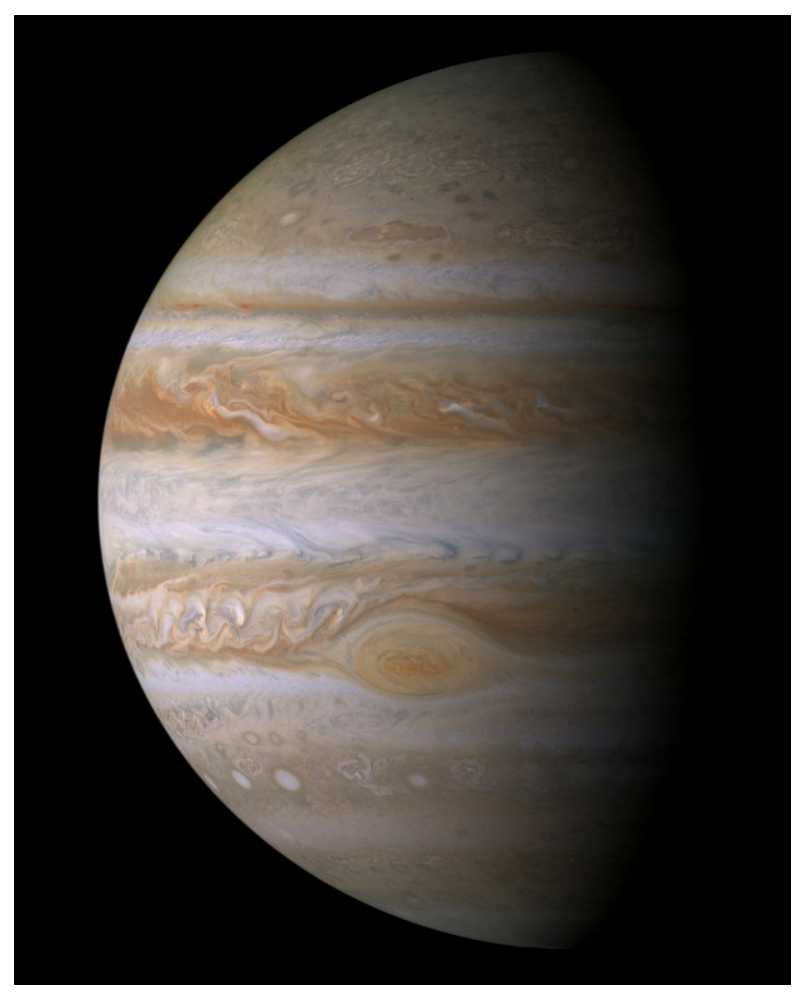

seasonal swings. A 70-day movie shows the clouds in motion at all longitudes and latitudes up to within $\sim 10$ degrees of the poles. That movie in cylindrical and polar projections is PIA03452, PIA03453, and PIA03454, and is available at https://photojournal.jpl.nasa.gov/.

The GRS is at least 150 years old, although it could be much older. The three white ovals to the south of the GRS formed in the late 1930s and merged into one oval named BA in the late 1990s. All of these features are anticyclones with circumferential winds greater than $100 \mathrm{~ms}^{-1}$. They drift slowly in longitude but stay fixed in latitude. Combined with Hubble Space Telescope and Galileo data, Cassini data were used to show that the GRS shrank by $15 \%$ from 1996 to 2006, both in terms of its visible appearance and its ring of circumferential winds (Asay-Davis et al. 2009; Shetty and Marcus 2010).

The white ovals occupied a single latitude band with anticyclonic vorticity. They avoided each other for 60 years because they were separated by cyclonic regions encroaching into the band from the equatorward side (Youssef and Marcus 2003). When the cyclonic regions got pushed out, the ovals merged. Peak velocities around oval BA remained steady while the color changed from white to red several years later. Using different techniques to measure winds and different definitions of the oval's edge, different groups have documented small changes in the properties of BA over similar time periods (Hueso et al. 2009; Choi et al. 2010; Sussman et al. 2010). The combination of Cassini ISS data and Hubble methane band data was used to understand the changes in vertical cloud structure as BA changed from white to red (Wong et al. 2011). Using ISS data from the Cassini flyby (Li et al. 2004; Choi et al. 2013) and amateur telescopic data (Rogers et al. 2006), several authors have documented the life cycles_formation, shape, lifetime, mergers-of the more numerous smaller spots on Jupiter. 
Fig. 19 Atmospheric zonal velocity for Jupiter. The black curve is from Cassini ISS data in late 2000, and the red curve is from Voyager data in mid-1979. The jets are remarkably steady over this 21-year interval (Porco et al. 2003)

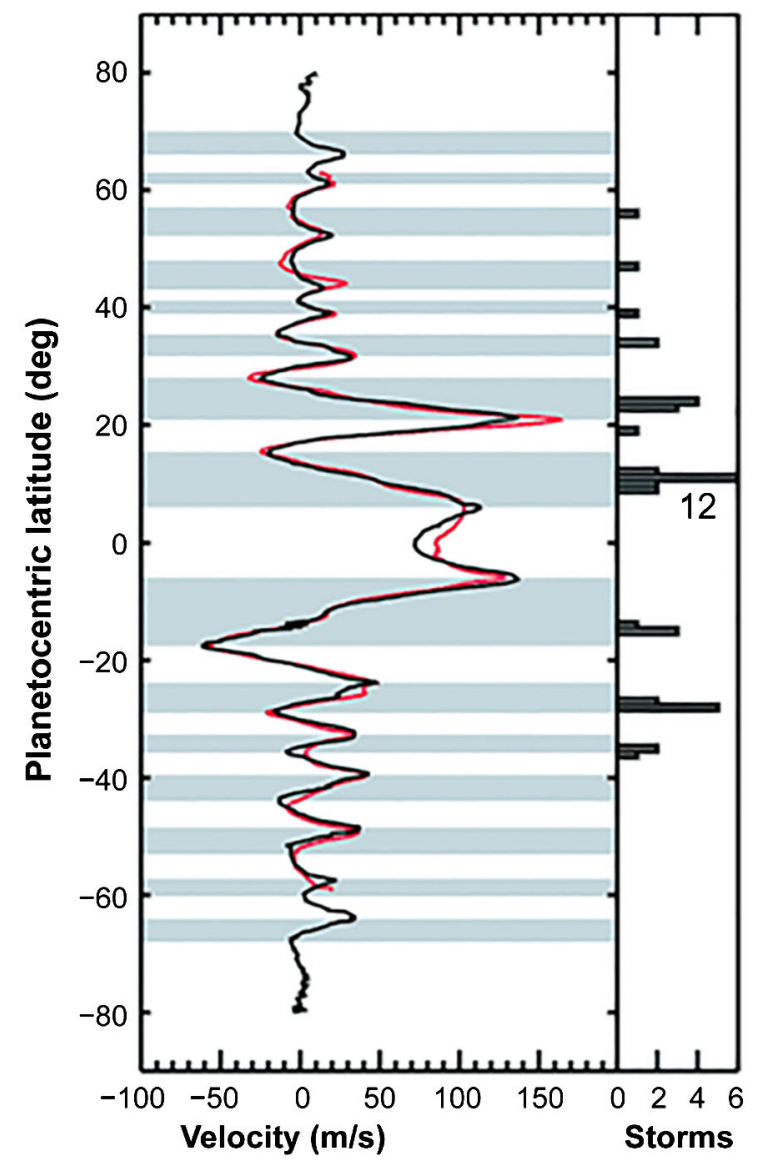

Jupiter's zonal jets are remarkably steady in comparison with Earth's jet streams, which change on time scales of 1 or 2 weeks (Fig. 19). Cassini ISS documented only one latitude outside the equator, near $21^{\circ}$ planetocentric, where the jet speed had changed by a modest amount from Voyager in 1979 to Cassini in 2000 (Porco et al. 2003; Asay-Davis et al. 2011). Most of the observed variability is in the stratosphere at the equator (Garcia-Melendo et al. 2011a) and is part of a regular $\sim 4$ year oscillation similar to the quasi-biennial oscillation on Earth (Flasar et al. 2004; Simon-Miller et al. 2007; Simon-Miller and Gierasch 2010). Meridional transport is inferred indirectly from Cassini CIRS data that give the latitudinal distribution of $\mathrm{C}_{2} \mathrm{H}_{2}$ and $\mathrm{C}_{2} \mathrm{H}_{6}$, which have known chemical lifetimes and thereby track the age of air masses in the stratosphere (Liang et al. 2005; Zhang et al. 2013b). Vertical wind shear of the zonal winds is studied by tracking features at different wavelengths that probe different altitudes (Li et al. 2006c). Cassini data.

The alternating white and grey bands in Fig. 19 are the zones and belts, respectively. The zones are defined as having anticyclonic vorticity (clockwise in the northern hemisphere) and the belts as having cyclonic vorticity (counterclockwise in the northern hemisphere). The vertical strip on the right shows the latitudes where bright white storm clouds associated with lightning occurred (Dyudina et al. 2004), and that was usually in the belts. Lighting in the belts has been a puzzle since the Galileo days (Little et al. 1999; Gierasch et al. 2000) because except for the lightning storms, the belts are generally clear and the zones 


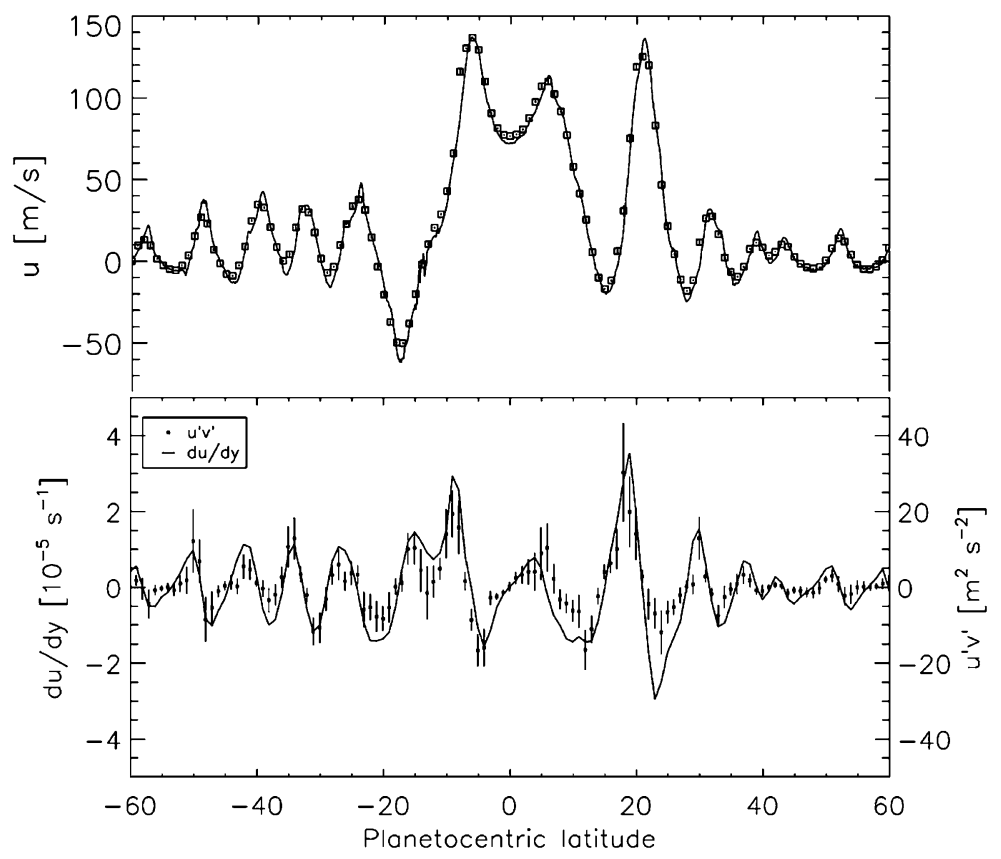

Fig. 20 Eddy momentum transport for Jupiter from Cassini ISS. The eastward and northward eddy winds $\mathrm{u}^{\prime}$ and $\mathrm{v}^{\prime}$ are the departures from the zonal means. Their product $\overline{u^{\prime} v^{\prime}}$ averaged over longitude and multiplied by density is the northward eddy flux of eastward momentum. The fact that this quantity has the same sign as $\partial \bar{u} / \partial y$, which is the increase of the mean eastward wind with latitude, says that the eddies are putting energy into the jets and not the reverse (Salyk et al. 2006)

are cloudy. Thus clouds and chemical tracers imply upwelling in the zones (Gierasch et al. 1986), but lightning and the eddy momentum transport imply the opposite. Figure 20 shows that the eddy momentum transport is into the jets (Salyk et al. 2006). To balance the angular momentum, there must be a mean north-south flow that is converging in the zones and diverging in the belts (Ingersoll et al. 2000), implying upwelling in the belts, consistent with lightning. So there is conflicting evidence of upwelling and downwelling. The Cassini data shown in Figs. 19 and 20 have not solved this contradiction, but they have at least focused attention on it (Fletcher et al. 2020). The double Hadley cell suggested for Saturn in Fig. 2 may be the key for Jupiter as well.

Waves in a planetary atmosphere provide information about the medium in which they propagate and also about the sources that excite them. Mesoscale waves-wavelengths $\sim 100 \mathrm{~km}$ - are visible from space if the crests and troughs are marked by clouds. The thunder following a lightning strike and the sonic boom following a meteor impact are examples of intense sound waves. Cassini could not detect sound waves, but it did detect thunderstorms and lightning (Dyudina et al. 2004; Porco et al. 2003; Baines et al. 2007). Voyager, Galileo, and New Horizons detected mesoscale wave trains with crests and troughs aligned north-south and wavelengths of $\sim 300 \mathrm{~km}$. Cassini apparently did not detect mesoscale waves (Arregi et al. 2009). They may have been absent at the time of the Cassini flyby or perhaps the waves were unobservable due to lack of cloud tracers. One theory says the mesoscale waves are propagating gravity waves (Flasar and Gierasch 1986), and another theory says they are shear instabilities (Bosak and Ingersoll 2002). The first theory requires an obstacle to the flow. The second theory requires strong shear and weak stratification. The 
measured phase velocities seem inconsistent with both models (Simon et al. 2015) given reasonable assumptions about the vertical wind shears, so the nature of the mesoscale waves is uncertain.

Cassini also detected large scale periodic patterns with wavelengths ranging up to $20,000 \mathrm{~km}$. Circumpolar waves have wavelengths and phase speeds that suggest they are Rossby waves (Barrado-Izagirre et al. 2008, 2009). Simultaneous observations by ISS, UVIS, and CIRS allow one to probe the vertical structure and opacity sources of the waves (Li et al. 2006b). Radio observations allow one to study how the passage of a wave affects the distribution of ammonia (Cosentino et al. 2017). Cassini observations have been used to support various theories of wave-like features in the South Equatorial Belt-that they are either inertia-gravity waves or Rossby waves (Simon-Miller et al. 2012) or a pattern associated with baroclinic instability at that latitude (Rogers et al. 2016).

Wave mean-flow interactions and cascades of energy from one scale to another are fundamental processes in the dynamics of planetary atmospheres. One theory says that the zonal mean flow forms into a potential vorticity (PV) staircase. PV is a conserved dynamical tracer and the staircase consists of latitude bands with constant PV and sharp boundaries in between. However, quantitative analysis of the Cassini 70-day movie shows that the PV gradient reverses sign and is not zero as the staircase model predicts ( $\mathrm{Li}$ et al. 2004; Read et al. 2006; Shetty and Marcus 2010). The 70-day movie was also used to show that the eddy momentum transport is into the jets from neighboring latitudes, meaning that the eddies are supplying energy to the jets and not the reverse (Fig. 20) (Salyk et al. 2006). The eddies could be getting their energy from convection ( $\mathrm{Li}$ et al. 2006a) or the latitudinal gradient of radiative heating, or both (Schneider and Liu 2009). Fourier spectra of the 70-day movie data generally reveal an inverse energy cascade, where kinetic energy flows from intermediate scales to large scales (Choi and Showman 2011; Galperin et al. 2014; Hadjighasem and Haller 2016), but there is evidence of a forward cascade from intermediate scales down to small scales, which has been interpreted as energy input at the intermediate scales, perhaps associated with the radius of deformation through baroclinic instability (Young and Read 2017). Others have compared visible features in the 70-day movie with fine-scale features in their 3-dimensional numerical models to test the models' treatment of unknown processes and parameters (Heimpel et al. 2005, 2016; Morales-Juberias and Dowling 2013).

\subsection{Atmospheric Structure and Composition}

Reactive gases can serve as tracers that reveal regions of upwelling, downwelling, and meridional motion (Fig. 21). During the Jupiter flyby, Cassini CIRS measured the latitude distributions of $\mathrm{C}_{2} \mathrm{H}_{2}$ and $\mathrm{C}_{2} \mathrm{H}_{6}$, which are produced in the upper stratosphere by photodissociation of methane. The rate of production is greatest at the equator where the solar ultraviolet (UV) is greatest. $\mathrm{C}_{2} \mathrm{H}_{2}$ has a chemical time constant that is short compared to the meridional overturning time, so it decreases toward the poles in response to the reduced solar UV. $\mathrm{C}_{2} \mathrm{H}_{6}$ has a much longer chemical time constant than $\mathrm{C}_{2} \mathrm{H}_{2}$ - many hundreds vs. tens of years depending on altitude. One might expect it to have a flat distribution if it were carried poleward faster than the chemical reaction rate. But its abundance increases toward the poles (Nixon et al. 2007, 2010), and that is more difficult to explain (Hue et al. 2018). It is possible to fit the $\mathrm{C}_{2} \mathrm{H}_{6}$ distribution by adjusting parameters of the meridional circulation, but then the $\mathrm{C}_{2} \mathrm{H}_{2}$ distribution doesn't fit. Independent estimates of the meridional circulation come from the distributions of $\mathrm{HCN}$ and $\mathrm{CO}_{2}$, which were measured by CIRS (Lellouch et al. 2006), but the problem remains. 

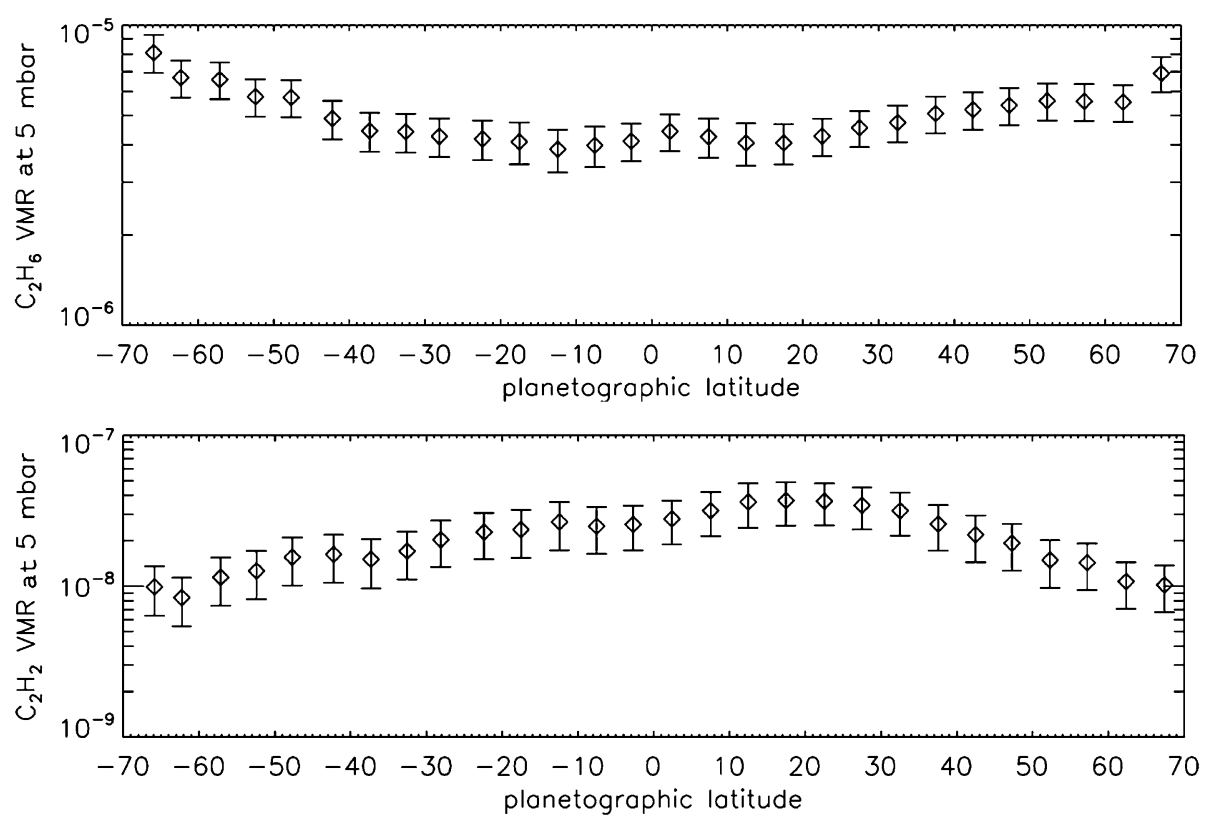

Fig. 21 Chemical tracers in Jupiter's atmosphere at 5 mbar from Cassini CIRS. Both acetylene and ethane are formed at low latitudes by solar UV. In photochemical equilibrium their abundances would be greatest at pressures less than 0.1 mbar. $\mathrm{C}_{2} \mathrm{H}_{2}$ has a short chemical time constant, $\sim 3 \times 10^{7} \mathrm{~s}$, so its abundance reflects photochemical equilibrium with lower sunlight toward the poles. $\mathrm{C}_{2} \mathrm{H}_{6}$ has a long lifetime, $\sim 3 \times 10^{10} \mathrm{~s}$, so its abundance at 5 mbar reflects transport by the meridional circulation. The time constant of this circulation is between the lifetimes of the two molecules (Nixon et al. 2007)

Cassini CIRS also measured the latitude distributions of phosphine (Irwin et al. 2004; Fletcher et al. 2009a) and ammonia (Achterberg et al. 2006). The measurements refer to the upper troposphere, at the 100-500 mbar level, and they tell a consistent story. Both gases are removed from the atmosphere at upper levels, phosphine by photodissociation and ammonia by condensation, and both have maximum abundance at the equator and reduced abundance in the cyclonic belts on either side of the equator. This indicates rapid uplift or strong vertical mixing in the equatorial zone and descent in the neighboring belts. Probing still deeper into the troposphere, Cassini VIMS detected a $3 \mu \mathrm{m}$ absorption that could be a mixture of $\mathrm{NH}_{4} \mathrm{SH}$ and $\mathrm{NH}_{3}$ (Brown et al. 2003; Sromovsky and Fry 2010) and provided new insights into the composition of fresh ammonia clouds (Sromovsky and Fry 2018).

Since the stratosphere is stably stratified, to maintain steady state the updrafts must be heated and the downdrafts must be cooled. The decrease of sunlight toward the poles is consistent with updrafts at the equator and downdrafts at the poles. CIRS measurements of stratospheric temperatures and abundances of radiatively active gases are used to model the radiative forcing (Zhang et al. 2013a, 2013b), which includes the effects of stratospheric aerosols (Zhang et al. 2013c, 2015).

Gaseous abundances can provide clues to planet formation. CIRS measured the nitrogen isotopic ratio in ammonia (Abbas et al. 2004; Fouchet et al. 2004a). The ratio is similar to that on the Sun but different from that on Earth, suggesting that the Earth was somehow isolated from the main reservoir of nitrogen during solar system formation. The hydrogen halides, on the other hand, are depleted relative to solar composition and support the halogens' condensation in ammonium salts (Fouchet et al. 2004b). 
The Cassini flyby provided insights into aurora-related phenomena on Jupiter as well. UVIS observations of the He $584 \AA$ line were used to infer enhanced eddy mixing in the polar regions, which may account for the enhancement of heavy hydrocarbons there (Parkinson et al. 2006). Alternately, the enhancement of $\mathrm{C}_{2} \mathrm{H}_{2}$ and $\mathrm{C}_{2} \mathrm{H}_{6}$ in the polar regions could be explained by a combination of auroral-driven chemistry and advection by the meridional circulation (Sinclair et al. 2017, 2019). CIRS observations have also been used to study the sources of heating in the polar stratosphere from 1-10 mbar (Sinclair et al. 2018). The result is that heating by auroral soot particles dominates at 1 mbar altitude, and that heating by charged particles dominates at $10 \mu$ bar. Cassini VIMS observations have been used to produce the first maps of $\mathrm{H}_{3}{ }^{+}$emission, temperature, and column density on Jupiter's nightside (Stallard et al. 2015). The enhanced emission in the polar regions is produced by enhanced $\mathrm{H}_{3}{ }^{+}$density rather than higher temperatures, which further points to the importance of auroral chemistry in that region.

Because of its high spatial and spectral resolution and full angular coverage during the flyby, Cassini obtained useful information about the clouds of Jupiter. Cloud color is a longstanding uncertainty: What chemical gives the Red Spot its redness? How many coloring agents are there? Comparison of laboratory experiments and visible spectra from Cassini VIMS suggests that the red chromophore is the product of photolyzed ammonia reacting with acetylene (Carlson et al. 2016; Baines et al. 2019). The chromophore occurs in an optically thin layer above the main cloud deck, and may require upward displacement of the air mass to form. A principal component analysis of Cassini ISS data indicates that the red chromophore explains most of the variance across the planet, but a second coloring agent is required to explain the brownish color of the cyclones in Jupiter's North Equatorial Belt (Ordonez-Etxeberria et al. 2016; Teifel' et al. 2018).

The vertical structure of the cloud layers is another long-standing unknown. Cassini CIRS thermal spectra were used to probe into the clouds at a wavelength of $7.18 \mu \mathrm{m}$, where the ammonia gas is relatively transparent (Matcheva et al. 2005). The result is that cloud base is at $0.9-1.1$ bar, which is deeper than the nominal ammonia cloud base and might imply another, deeper cloud, possibly $\mathrm{NH}_{4} \mathrm{SH}$. Cassini VIMS covers 4.5-5.1 $\mu \mathrm{m}$, another wavelength range where the gas is relatively transparent. Holes in the cloud allow thermal radiation to space, so the holes appear as hot spots. The cloud is found to be spectrally flat and at a pressure of 1.2 bar or deeper (Giles et al. 2015). Pure $\mathrm{NH}_{3}$ cloud and pure $\mathrm{NH}_{4} \mathrm{SH}$ cloud do not match the spectra, and some kind of coating is implied. Despite the possibility of coatings, CIRS has detected the spectral signature of $\mathrm{NH}_{3}$ ice particles in the upper tropospheric clouds (Wong et al. 2004).

The planet's global energy budget is a key to its evolution and internal processes, including the separation of helium from hydrogen in the metallic interior. To determine the energy budget accurately, one has to measure the thermal emission and scattered sunlight at all seasons, latitudes, longitudes, wavelengths, emission angles, incident angles, and phase angles. In practice, one samples these quantities and uses models to fill the gaps. Cassini CIRS, VIMS, and ISS all contribute to this estimate (Simon-Miller et al. 2006; Li et al. 2012; Sato et al. 2013; Mayorga et al. 2016). A comprehensive recent study (Li et al. 2018) reveals that Jupiter's Bond albedo and internal heat, $0.503 \pm 0.012$ and $7.4785 \pm 0.16 \mathrm{~W} \mathrm{~m}^{-2}$ are both $\sim 40 \%$ larger than the previous best estimates. The higher luminosity might come from greater helium differentiation, such that Saturn has formed a deep helium-rich shell or core (Mankovich and Fortney 2020). 


\section{Venus Atmosphere}

The Venus flybys yielded useful data at a variety of wavelengths. The VIMS instrument detected thermal emission from the hot surface at wavelengths of 0.85 and $0.90 \mu \mathrm{m}$, and thereby provided a new technique for exploring the surface mineralogy (Baines et al. 2000). In an attempt to image the surface, the RADAR instrument was operated at $2.2 \mathrm{~cm}$ in both scatterometric and radiometric modes during the encounters, but only the thermal emission from the thick absorbing atmosphere was detected (Lorenz et al. 2001). The UVIS instrument studied the airglow of Venus, focusing on $\mathrm{N}_{2}, \mathrm{C}, \mathrm{O}$, and $\mathrm{CO}$ as clues to the excitation mechanisms and abundances (Hubert et al. 2010, 2012; Gerard et al. 2011). Lightning on Venus is a long-standing controversial subject. The RPWS instrument on Cassini searched for lightning signals in the frequency range 0.125 to $16 \mathrm{MHz}$ and found none, even though they easily detected lightning at rates of $70 \mathrm{~s}^{-1}$ during the Earth flyby several months later (Gurnett et al. 2001).

\section{Open Science Questions After Cassini}

For an object as complex as Saturn, there will always be unanswered questions following a successful mission, and Cassini is no exception. Here is a list, by no means complete, of objectives that seem possible with future missions and expanded technologies.

Water Measure the global water abundance and determine its role in bringing heat to the surface. Determine the role of moist convection in maintaining the large-scale motions. A Saturn probe to the base of the water cloud is a challenging but possible objective. Juno-type microwave remote sensing, in addition, is possible and has the advantage of global coverage.

Ammonia Determine the global ammonia abundance, and map its distribution with depth over the planet. Currently available data are good down to only about 3 bars, which do not necessarily represent the deep well mixed abundance of ammonia. A Saturn probe to 10 bars could make this measurement, and microwave remote sensing is potentially valuable.

Noble Gases Determine Saturn's noble gas abundances including helium, and their isotopic ratios, as done for Jupiter by the Galileo probe. A Saturn probe could measure noble gases to exquisite precision.

Weather Layer Determine the vertical profiles of temperature, winds, clouds, condensable gases, longwave and shortwave radiation, and turbulence levels. Again, a Saturn probe is needed. A cloud-resolving model like those used in tropical meteorology, but tailored to the giant planets, is both timely and valuable.

Interior Motions Determine Saturn's rotation period if such a number exists, or determine the spread of periods if differential motions persist into the interior. Document the time dependence of motions in Saturn's interior.

Interior Structure Using the power of ring seismology and gravity sounding, improve the understanding of Saturn's internal equation of state, mass distribution, composition, and temperature distribution. Develop a fully coupled interior/weather-layer model as done for Earth's ocean/atmosphere system. 


\section{Scientific Objectives}

This section gives the Saturn portion of the scientific objectives for the prime mission and for the Cassini Solstice Mission, which was a mission extension. The former are listed either as S_AOn or J_AOn, with S for Saturn, J for Jupiter, AO for announcement of opportunity and $\mathrm{n}$ as a counter in the list. The latter are listed as SNnx and SCny, with S for Saturn, N for new phenomena to pursue, and $\mathrm{C}$ for change, both intrinsic and seasonally driven. Again $\mathrm{nx}$ and ny are counters. The list below is reproduced from Volume 1 of the Cassini Mission final report to NASA; Sect. 3, Science mission results; Saturn discipline science results. The full document is available at https://pds-rings.seti.org/cassini/.

- Saturn Temperature, Clouds, Composition (S_AO1)

- Temperature and clouds (S_AO1) - Determine temperature field, cloud properties, and composition of the atmosphere of Saturn.

- Composition and chemistry (SN1c) - Measure the spatial and temporal variability of trace gases and isotopes.

- Saturn Winds and Weather (S_AO2)

- Seasonal Variations (SC1a) - Observe seasonal variations in temperature, clouds, and composition in three spatial dimensions.

- Saturn's Winds (SC1b) - Observe seasonal changes in the winds at all accessible altitudes coupled with simultaneous observations of clouds, temperatures, composition, and lightning.

- Storms (SN1b, SN2a) - Observe the aftermath of the 2010-2011 storm. Study the life cycles of Saturn's newly discovered atmospheric waves, south polar hurricane, and rediscovered north polar hexagon. Monitor the planet for new storms and respond with observations when they occur.

- Atmospheric dynamical processes (S_AO2) - Measure the global wind field, including wave and eddy components; observe synoptic cloud features and processes.

- Saturn Lightning Sources and Morphology (S_AO6, SN2a) - Investigate the sources and the morphology of Saturn lightning-Saturn Electrostatic Discharges (SED) lightning whistlers.

- Saturn Interior Structure and Rotation (S_AO3)

- Saturn's Rotation Rate (SN1a) - Determine Saturn's rotation rate and internal structure despite the planet's unexpected high degree of axisymmetry.

- Saturn Formation and Evolution (S_AO5) - Provide observational constraints (gas composition, isotope ratios, heat flux, ...) on scenarios for the formation and the evolution of Saturn.

- Aurora, Chemistry, and Upper Atmosphere (SC2a) - Observe the upper atmosphere and the aurora as it changes on all time scales-minutes to years-and is affected by seasonal and solar cycle forcing.

- Jupiter atmospheric dynamics (J_AO1) - Extend the time for studies of atmospheric dynamics beyond the period accessible to the Galileo nominal mission.

- Jupiter Global Atmospheric Structure and Composition (J_AO2) - Infer global atmospheric thermal structure and composition with instrumentation not carried by the Galileo Orbiter, complementing the local in situ measurements of the Galileo Probe.

- Venus atmosphere observations - No specific objectives were called out.

- Cruise Planetary and Stellar Internal Oscillations (C_AO5) - Attempt to detect internal oscillations of Saturn, Jupiter, and some stars.

- Saturn Ionosphere-Magnetospheric Interaction (S_AO4) - Study the diurnal variations and magnetic control of the ionosphere of Saturn. 


\section{Acronyms}

$\begin{array}{ll}\text { AO } & \text { Announcement of Opportunity } \\ \text { CIRS } & \text { Composite Infrared Spectrometer } \\ \text { GCM } & \text { general circulation model } \\ \text { GRS } & \text { Great Red Spot } \\ \text { HST } & \text { Hubble Space Telescope } \\ \text { IR } & \text { infrared } \\ \text { ISS } & \text { Imaging Science Subsystem } \\ \text { MAPS } & \text { Magnetospheres and Plasma Science } \\ \text { PV } & \text { potential vorticity } \\ \text { QBO } & \text { quasi-biennial oscillation } \\ \text { RPWS } & \text { Radio and Plasma Wave Science } \\ \text { SAO } & \text { Semiannual Oscillation } \\ \text { SED } & \text { Saturn Electrostatic Discharges } \\ \text { SKR } & \text { Saturn kilometric radiation } \\ \text { UVIS } & \text { Ultraviolet Imaging Spectrograph } \\ \text { VIMS } & \text { Visual and Infrared Mapping Spectrometer }\end{array}$

Acknowledgements This research was supported by Grant NAS7-03001, Cassini Project, NASA Jet Propulsion Laboratory, USA. I thank my Cassini colleagues for their many scientific contributions and their ability to work together in guiding this mission. Scott Edgington and Linda Spilker were extremely helpful as I prepared this document. It was a pleasure working on this great mission. The lesson is, if you build a well-equipped spacecraft and send it to an unexplored place, the discoveries will come.

Funding information Grant NAS7-03001, Cassini Project, NASA Jet Propulsion Laboratory, USA.

Data availability Cassini data can be accessed on the Planetary Data System (PDS) at https://pds.nasa.gov.

Code availability Not applicable.

Conflict of interest None.

Publisher's Note Springer Nature remains neutral with regard to jurisdictional claims in published maps and institutional affiliations.

Open Access This article is licensed under a Creative Commons Attribution 4.0 International License, which permits use, sharing, adaptation, distribution and reproduction in any medium or format, as long as you give appropriate credit to the original author(s) and the source, provide a link to the Creative Commons licence, and indicate if changes were made. The images or other third party material in this article are included in the article's Creative Commons licence, unless indicated otherwise in a credit line to the material. If material is not included in the article's Creative Commons licence and your intended use is not permitted by statutory regulation or exceeds the permitted use, you will need to obtain permission directly from the copyright holder. To view a copy of this licence, visit http://creativecommons.org/licenses/by/4.0/.

\section{References}

M.M. Abbas, A. LeClair, T. Owen, B.J. Conrath, F.M. Flasar, V.G. Kunde et al., The nitrogen isotopic ratio in Jupiter's atmosphere from observations by the Composite Infrared Spectrometer on the Cassini spacecraft. Astrophys. J. 602, 1063-1074 (2004). https://doi.org/10.1086/381084

M.M. Abbas et al., Distribution of $\mathrm{CO}_{2}$ in Saturn's atmosphere from Cassini/CIRS infrared observations. Astrophys. J. 776(2), 73 (2013). https://doi.org/10.1088/0004-637x/776/2/73

R.K. Achterberg, B.J. Conrath, P.J. Gierasch, Cassini CIRS retrievals of ammonia in Jupiter's upper troposphere. Icarus 182, 169-180 (2006). https://doi.org/10.1016/j.icarus.2005.12.020 
R.K. Achterberg, P.J. Gierasch, B.J. Conrath, L.N. Fletcher, B.E. Hesman, G.L. Bjoraker, F.M. Flasar, Changes to Saturn's zonal-mean tropospheric thermal structure after the 2010-2011 northern hemisphere storm. Astrophys. J. 786(2), 92 (2014). https://doi.org/10.1088/0004-637x/786/2/92

R.K. Achterberg, F.M. Flasar, G.L. Bjoraker, B.E. Hesman, N.J.P. Gorius, A.A. Mamoutkine et al., Thermal emission from Saturn's polar cyclones. Geophys. Res. Lett. 45(11), 5312-5319 (2018). https://doi.org/ 10.1029/2018GL078157

A. Adriani, M.L. Moriconi, E. D’Aversa, F. Oliva, G. Filacchione, Faint luminescent ring over Saturn's polar hexagon. Astrophys. J. 808, L16 (2015). https://doi.org/10.1088/2041-8205/808/1/16

A.C.B. Aguiar, P.L. Read, R.D. Wordsworth, T. Salter, Y.H. Yamazaki, A laboratory model of Saturn's north polar hexagon. Icarus 206(2), 755-763 (2010). https://doi.org/10.1016/j.icarus.2009.10.022

J.D. Anderson, G. Schubert, Saturn's gravitational field, internal rotation, and interior structure. Science 317, 1384-1387 (2007). https://doi.org/10.1126/science.1144835

A. Antunano, T. del Rio-Gaztelurrutia, A. Sanchez-Lavega, R. Hueso, Dynamics of Saturn's polar regions. J. Geophys. Res., Planets 120(2), 155-176 (2015). https://doi.org/10.1002/2014je004709

A. Antunano, T. del Rio-Gaztelurrutia, A. Sanchez-Lavega, J. Rodriguez-Aseguinolaza, Cloud morphology and dynamics in Saturn's northern polar region. Icarus 299, 117-132 (2018). https://doi.org/10.1016/j. icarus.2017.07.017

A. Antunano, T. del Río-Gaztelurrutia, A. Sánchez-Lavega, P.L. Read, L.N. Fletcher, Potential vorticity of Saturn's polar regions. J. Geophys. Res. 124, 186-201 (2019). https://doi.org/10.1029/2018JE005764

J. Arregi, J.F. Rojas, R. Hueso, A. Sanchez-Lavega, Gravity waves in Jupiter's equatorial clouds observed by the Galileo orbiter. Icarus 202, 358-360 (2009). https://doi.org/10.1016/j.icarus.2009.03.028

X.S. Asay-Davis, P.S. Marcus, M.H. Wong, I. de Pater, Jupiter's shrinking Great Red Spot and steady Oval BA: Velocity measurements with the "Advection Corrected Correlation Image Velocimetry" automated cloud-tracking method. Icarus 203, 164-188 (2009). https://doi.org/10.1016/j.icarus.2009.05.001

X.S. Asay-Davis, P.S. Marcus, M.H. Wong, I. de Pater, Changes in Jupiter's zonal velocity between 1979 and 2008. Icarus 211, 1215-1232 (2011). https://doi.org/10.1016/j.icarus.2010.11.018

S.K. Atreya, M.H. Wong, T.C. Owen, P.R. Mahaffy, H.B. Niemann, I. de Pater, P. Drossart, T. Encrenaz, A comparison of the atmospheres of Jupiter and Saturn: deep atmospheric composition, cloud structure, vertical mixing, and origin. Planet. Space Sci. 47(10-11), 1243-1262 (1999). https://doi.org/10.1016/ s0032-0633(99)00047-1.

S.K. Atreya, P.R. Mahaffy, H.B. Niemann, M.H. Wong, T.C. Owen, Composition and origin of the atmosphere of Jupiter - an update, and implications for the extrasolar giant planets. Planet. Space Sci. 51(2), 105-112 (2003). https://doi.org/10.1016/s0032-0633(02)00144-7

S.K. Atreya, A. Crida, T. Guillot, J.I. Lunine, N. Madhusudhan, M. Mousis, The origin and evolution of Saturn, with exoplanet perspective, in Saturn in the 21 st Century, ed. by K. Baines, M. Flasar, N. Krupp, T. Stallard (Cambridge University Press, Cambridge, 2019), pp. 5-43

J. Aurnou, M. Heimpel, L. Allen, E. King, J. Wicht, Convective heat transfer and the pattern of thermal emission on the gas giants. Geophys. J. Int. 173(3), 793-801 (2008). https://doi.org/10.1111/j.1365246X.2008.03764.x

J.V. Auwera, N. Moazzen-Ahmadi, J.M. Flaud, Toward an accurate database for the $12 \mu \mathrm{m}$ region of the ethane spectrum. Astrophys. J. 662(1), 750-757 (2007)

K.H. Baines, G. Bellucci, J.P. Bibring, R.H. Brown, B.J. Buratti, E. Bussoletti et al., Detection of sub-micron radiation from the surface of Venus by Cassini/VIMS. Icarus 148, 307-311 (2000). https://doi.org/10. 1006/icar.2000.6519

K.H. Baines et al., The atmospheres of Saturn and Titan in the near-infrared: first results of Cassini/VIMS. Earth Moon Planets 96, 3-4, 119-147 (2005). https://doi.org/10.1007/s11038-005-9058-2

K.H. Baines, A.A. Simon-Miller, G.S. Orton, H.A. Weaver, A. Lunsford, T.W. Momary et al., Polar lightning and decadal-scale cloud variability on Jupiter. Science 318, 226-229 (2007). https://doi.org/10.1126/ science. 1147912

K.H. Baines, M.L. Delitsky, T.W. Momary, R.H. Brown, B.J. Buratti, R.N. Clark, P.D. Nicholson, Storm clouds on Saturn: lightning-induced chemistry and associated materials consistent with Cassini/VIMS spectra. Planet. Space Sci. 57(14-15), 1650-1658 (2009a). https://doi.org/10.1016/j.pss.2009.06.025

K.H. Baines, T.W. Momary, L.N. Fletcher, A.P. Showman, M. Roos-Serote, R.H. Brown, B.J. Buratti, R.N. Clark, P.D. Nicholson, Saturn's north polar cyclone and hexagon at depth revealed by Cassini/VIMS. Planet. Space Sci. 57(14-15), 1671-1681 (2009b). https://doi.org/10.1016/j.pss.2009.06.026

K.H. Baines, L.A. Sromovsky, P.M. Fry, T.W. Momary, R.H. Brown, B.J. Buratti, R.N. Clark, P.D. Nicholson, C. Sotin, The eye of Saturn's north polar vortex: unexpected cloud structures observed at high spatial resolution by Cassini/VIMS. Geophys. Res. Lett. 45, 5867-5875 (2018)

K.H. Baines, L.A. Sromovsky, R.W. Carlson, T.W. Momary, P.M. Fry, The visual spectrum of Jupiter's Great Red Spot accurately modeled with aerosols produced by photolyzed ammonia reacting with acetylene. Icarus 330, 217-229 (2019) 
D. Banfield, B.J. Conrath, P.J. Gierasch, P.D. Nicholson, K. Matthews, Near-IR spectrophotometry of Jovian aerosols - meridional and vertical distributions. Icarus 134(1), 11-23 (1998). https://doi.org/10.1006/ icar.1998.5942

N. Barrado-Izagirre, A. Sanchez-Lavega, S. Perez-Hoyos, R. Hueso, Jupiter's polar clouds and waves from Cassini and HST images: 1993-2006. Icarus 194, 173-185 (2008). https://doi.org/10.1016/j.icarus.2007. 08.025

N. Barrado-Izagirre, S. Perez-Hoyos, A. Sanchez-Lavega, Brightness power spectral distribution and waves in Jupiter's upper cloud and hazes. Icarus 202, 181-196 (2009). https://doi.org/10.1016/j.icarus.2009. 02.015

J.K. Barstow, P.G.J. Irwin, L.N. Fletcher, R.S. Giles, C. Merlet, Probing Saturn's tropospheric cloud with Cassini/VIMS. Icarus 271, 400-417 (2016). https://doi.org/10.1016/j.icarus.2016.01.013

R.F. Beebe, Comparative study of the dynamics of the outer planets. Space Sci. Rev. 116(1-2), 137-154 (2005). https://doi.org/10.1007/s11214-005-1952-4

T. Bosak, A.P. Ingersoll, Shear instabilities as a probe of Jupiter's atmosphere. Icarus 158(2), 401-409 (2002). https://doi.org/10.1006/icar.2002.6886

R.H. Brown, K.H. Baines, G. Bellucci, J.P. Bibring, B.J. Buratti, F. Capaccioni et al., Observations with the Visual and Infrared Mapping Spectrometer (VIMS) during Cassini's flyby of Jupiter. Icarus 164, 461-470 (2003). https://doi.org/10.1016/s0019-1035(03)00134-9

Z. Brown, T. Koskinen, I. Muller-Wodarg, R. West, A. Jouchoux, L. Esposito, A pole-to-pole pressuretemperature map of Saturn's thermosphere from Cassini Grand Finale data. Nat. Astron. 4, 872-879 (2020). https://doi.org/10.1038/s41550-020-1060-0

S.B. Brueshaber, K.M. Sayanagi, T.E. Dowling, Dynamical regimes of giant planet polar vortices. Icarus 323, 46-61 (2019)

E.J. Bunce et al., Cassini nightside observations of the oscillatory motion of Saturn's northern auroral oval. J. Geophys. Res. Space Phys. 119(5), 3528-3543 (2014). https://doi.org/10.1002/2013ja019527

H. Cao, M.K. Dougherty, G.J. Hunt, G. Provan, S.W.H. Cowley, E.J. Bunce, S. Kellock, D.J. Stevenson, The landscape of Saturn's internal magnetic field from the Cassini Grand Finale. Icarus 344, UNSP 113541 (2020)

R.W. Carlson, K.H. Baines, M.S. Anderson, G. Filacchione, A.A. Simon, Chromophores from photolyzed ammonia reacting with acetylene: application to Jupiter's Great Red Spot. Icarus 274, 106-115 (2016). https://doi.org/10.1016/j.icarus.2016.03.008

T. Cavalie, M. Dobrijevic, L.N. Fletcher, J.C. Loison, K.M. Hickson, V. Hue, P. Hartogh, Photochemical response to the variation of temperature in the 2011-2012 stratospheric vortex of Saturn. Astron. Astrophys. 580, A55 (2015). https://doi.org/10.1051/0004-6361/201425444

T. Cavalie, V. Hue, P. Hartogh, R. Moreno, E. Lellouch, H. Feuchtgruber, C. Jarchow, T. Cassidy, L.N. Fletcher, F. Billebaud, M. Dobrijevic, L. Rezac, G.S. Orton, M. Rengel, T. Fouchet, S. Guerlet, Herschel map of Saturn's stratospheric water, delivered by the plumes of Enceladus. Astron. Astrophys. 630, A87 (2019)

D.S. Choi, A.P. Showman, Power spectral analysis of Jupiter's clouds and kinetic energy from Cassini. Icarus 216, 597-609 (2011). https://doi.org/10.1016/j.icarus.2011.10.001

D.S. Choi, A.P. Showman, R.H. Brown, Cloud features and zonal wind measurements of Saturn's atmosphere as observed by Cassini/VIMS. J. Geophys. Res., Planets 114, E04007 (2009). https://doi.org/10.1029/ 2008je003254

D.S. Choi, A.P. Showman, A.R. Vasavada, The evolving flow of Jupiter's White Ovals and adjacent cyclones. Icarus 207, 359-372 (2010). https://doi.org/10.1016/j.icarus.2009.10.013

D.S. Choi, A.P. Showman, A.R. Vasavada, A.A. Simon-Miller, Meteorology of Jupiter's equatorial hot spots and plumes from Cassini. Icarus 223, 832-843 (2013). https://doi.org/10.1016/j.icarus.2013.02.001

U.R. Christensen, J. Wicht, W. Dietrich, Mechanisms for limiting the depth of zonal winds in the gas giant planets. Astrophys. J. 890, 61 (2020)

B.J. Conrath, D. Gautier, Saturn helium abundance: a reanalysis of Voyager measurements. Icarus 144(1), 124-134 (2000). https://doi.org/10.1006/icar.1999.6265

B.J. Conrath, D. Gautier, R.A. Hanel, J.S. Hornstein, The helium abundance of Saturn from Voyager measurements. Astrophys. J. 282(2), 807-815 (1984). https://doi.org/10.1086/162267

R.G. Cosentino, B. Butler, B. Sault, R. Morales-Juberias, A. Simon, I. de Pater, Atmospheric waves and dynamics beneath Jupiter's clouds from radio wavelength observations. Icarus 292, 168-181 (2017). https://doi.org/10.1016/j.icarus.2017.01.006

K. Cuff, M. Heimpel, Effects of neutral buoyancy outer boundary condition in models of deep convection in giant planets. Phys. Earth Planet. Inter. 282, 89-99 (2018)

E. Dederick, J. Jackiewicz, T. Guillot, An analysis of stochastic Jovian oscillation excitation by moist convection. Astrophys. J. 856, 50 (2018) 
A.D. Del Genio, J.M. Barbara, Constraints on Saturn's tropospheric general circulation from Cassini ISS images. Icarus 219(2), 689-700 (2012). https://doi.org/10.1016/j.icarus.2012.03.035

A.D. Del Genio, J.M. Barbara, An objective classification of Saturn cloud features from Cassini ISS images. Icarus 271, 222-236 (2016). https://doi.org/10.1016/j.icarus.2016.02.011

A.D. Del Genio, J.M. Barbara, J. Ferrier, A.P. Ingersoll, R.A. West, A.R. Vasavada, J. Spitale, C.C. Porco, Saturn eddy momentum fluxes and convection: first estimates from Cassini images. Icarus 189(2), 479492 (2007). https://doi.org/10.1016/j.icarus.2007.02.013

A.D. Del Genio, R.K. Achterberg, K.H. Baines, F.M. Flasar, P.L. Read, A. Sanchez-Lavega, A.P. Showman, Saturn atmospheric structure and dynamics, in Saturn from Cassini-Huygens, ed. by M.K. Doughterty, L.W. Esposito, S.M. Krimigis (Springer, Dordrecht, 2009), pp. 113-159

T. del Rio-Gaztelurrutia, J. Legarreta, R. Hueso, S. Perez-Hoyos, A. Sanchez-Lavega, A long-lived cyclone in Saturn's atmosphere: observations and models. Icarus 209(2), 665-681 (2010). https://doi.org/10. 1016/j.icarus.2010.04.002

T. del Rio-Gaztelurrutia, A. Sanchez-Lavega, A. Antunano, J. Legarreta, E. Garcia-Melendo, K.M. Sayanagi et al., A planetary-scale disturbance in a long living three vortex coupled system in Saturn's atmosphere. Icarus 302, 499-513 (2018). https://doi.org/10.1016/j.icarus.2017.11.029

M.D. Desch, M.L. Kaiser, Voyager measurement of the rotation period of Saturn's magnetic field. Geophys. Res. Lett. 8(3), 253-256 (1981). https://doi.org/10.1029/GL008i003p00253

V.M. Devi, I. Kleiner, R.L. Sams, L.R. Brown, D.C. Benner, L.N. Fletcher, Line positions and intensities of the phosphine $\left(\mathrm{PH}_{3}\right)$ pentad near $4.5 \mu \mathrm{m}$. J. Mol. Spectrosc. 298, 11-23 (2014). https://doi.org/10.1016/ j.jms.2014.01.0130022-2852

M. Dobrijevic, T. Cavalie, F. Billebaud, A methodology to construct a reduced chemical scheme for 2D-3D photochemical models: application to Saturn. Icarus 214(1), 275-285 (2011). https://doi.org/10.1016/ j.icarus.2011.04.027

M. Dougherty (Cassini magnetometer team), Cassini's magnetometer at Saturn. Astron. Geophys. 58(4), 3642 (2017). https://doi.org/10.1093/astrogeo/atx140

T.E. Dowling, Dynamics of Jovian atmospheres. Annu. Rev. Fluid Mech. 27, $293-334$ (1995). https://doi. org/10.1146/annurev.fl.27.010195.001453

D. Dubrovin, A. Luque, F.J. Gordillo-Vazquez, Y. Yair, F.C. Parra-Rojas, U. Ebert, C. Price, Impact of lightning on the lower ionosphere of Saturn and possible generation of halos and sprites. Icarus 241, 313-328 (2014). https://doi.org/10.1016/j.icarus.2014.06.025

U.A. Dyudina, A.D. Del Genio, A.P. Ingersoll, C.C. Porco, R.A. West, A.R. Vasavada, J.M. Barbara, Lightning on Jupiter observed in the $\mathrm{H}$-alpha line by the Cassini imaging science subsystem. Icarus 172, 24-36 (2004). https://doi.org/10.1016/j.icarus.2004.07.014

U.A. Dyudina, A.P. Ingersoll, S.P. Ewald, C.C. Porco, G. Fischer, W. Kurth, M. Desch, A. Del Genio, J. Barbara, J. Ferrier, Lightning storms on Saturn observed by Cassini ISS and RPWS during 2004-2006. Icarus 190(2), 545-555 (2007). https://doi.org/10.1016/j.icarus.2007.03.035

U.A. Dyudina et al., Dynamics of Saturn's south polar vortex. Science 319(5871), 1801 (2008). https:// doi.org/10.1126/science. 1153633

U.A. Dyudina et al., Saturn's south polar vortex compared to other large vortices in the Solar System. Icarus 202(1), 240-248 (2009). https://doi.org/10.1016/j.icarus.2009.02.014

U.A. Dyudina, A.P. Ingersoll, S.P. Ewald, C.C. Porco, G. Fischer, W.S. Kurth, R.A. West, Detection of visible lightning on Saturn. Geophys. Res. Lett. 37, L09205 (2010). https://doi.org/10.1029/2010g1043188

U.A. Dyudina, A.P. Ingersoll, S.P. Ewald, C.C. Porco, G. Fischer, Y. Yair, Saturn's visible lightning, its radio emissions, and the structure of the 2009-2011 lightning storms. Icarus 226(1), 1020-1037 (2013). https://doi.org/10.1016/j.icarus.2013.07.013

U.A. Dyudina, X. Zhang, L. Li, P. Kopparla, A.P. Ingersoll, L. Dones, A. Verbiscer, Y.L. Yung, Reflected light curves, spherical and bond albedos of Jupiter- and Saturn-like exoplanets. Astrophys. J. 822(2), 76 (2016). https://doi.org/10.3847/0004-637x/822/2/76

W.M. Farrell, M.L. Kaiser, G. Fischer, P. Zarka, W.S. Kurth, D.A. Gurnett, Are Saturn electrostatic discharges really superbolts? A temporal dilemma. Geophys. Res. Lett. 34, 6 (2007). https://doi.org/10.1029/ $2006 \mathrm{gl} 1028841$

B. Fegley, K. Lodders, Chemical models of the deep atmospheres of Jupiter and Saturn. Icarus 110(1), 117154 (1994). https://doi.org/10.1006/icar.1994.1111

G. Fischer et al., Saturn lightning recorded by Cassini/RPWS in 2004. Icarus 183(1), 135-152 (2006a). https://doi.org/10.1016/j.icarus.2006.02.010

G. Fischer, W. Macher, D.A. Gurnett, M.D. Desch, A. Lecacheux, P. Zarka, W.S. Kurth, M.L. Kaiser, Discrimination between Jovian radio emissions and Saturn electrostatic discharges. Geophys. Res. Lett. 33, 21 (2006b). https://doi.org/10.1029/2006gl026766

G. Fischer, W.S. Kurth, U.A. Dyudina, M.L. Kalser, P. Zarka, A. Lecacheux, A.P. Ingersoll, D.A. Gurnett, Analysis of a giant lightning storm on Saturn. Icarus 190(2), 528-544 (2007). https://doi.org/10.1016/j. icarus.2007.04.002 
G. Fischer, D.A. Gurnett, W.S. Kurth, F. Akalin, P. Zarka, U.A. Dyudina, W.M. Farrell, M.L. Kaiser, Atmospheric electricity at Saturn. Space Sci. Rev. 137(1-4), 271-285 (2008). https://doi.org/10.1007/ s11214-008-9370-z

G. Fischer et al., A giant thunderstorm on Saturn. Nature 475(7354), 75-77 (2011). https://doi.org/10.1038/ nature 10205

G. Fischer, S.Y. Ye, J.B. Groene, A.P. Ingersoll, K.M. Sayanagi, J.D. Menietti, W.S. Kurth, D.A. Gurnett, A possible influence of the Great White Spot on Saturn kilometric radiation periodicity. Ann. Geophys. 32(12), 1463-1476 (2014). https://doi.org/10.5194/angeo-32-1463-2014

F.M. Flasar, Temperatures, winds, and composition in the Saturnian system. Science 307(5713), 1247-1251 (2005). https://doi.org/10.1126/science.1105806

F. Flasar, P. Gierasch, Mesoscale waves as a probe of Jupiter's deep atmosphere. J. Atmos. Sci. 43(22), 26832707 (1986). https://doi.org/10.1175/1520-0469(1986)043<2683:MWAAPO>2.0.CO;2

F.M. Flasar, V.G. Kunde, R.K. Achterberg, B.J. Conrath, A.A. Simon-Miller, C.A. Nixon, P.J. Gierasch, P.N. Romani, B. Bezard, P. Irwin, G.L. Bjoraker, J.C. Brasunas, D.E. Jennings, J.C. Pearl, M.D. Smith, G.S. Orton, L.J. Spilker, R. Carlson, S.B. Calcutt, P.L. Read, F.W. Taylor, P. Parrish, A. Barucci, R. Courtin, A. Coustenis, D. Gautier, E. Lellouch, A. Marten, R. Prange, Y. Biraud, T. Fouchet, C. Ferrari, T.C. Owen, M.M. Abbas, R.E. Samuelson, F. Raulin, P. Ade, C.J. Cesarsky, K.U. Grossman, A. Coradini, An intense stratospheric jet on Jupiter. Nature 427, 132-135 (2004)

L.N. Fletcher, P.G.J. Irwin, N.A. Teanby, G.S. Orton, P.D. Parrish, S.B. Calcutt, N. Bowles, R. de Kok, C. Howett, F.W. Taylor, The meridional phosphine distribution in Saturn's upper troposphere from Cassini/CIRS observations. Icarus 188(1), 72-88 (2007a). https://doi.org/10.1016/j.icarus.2006.10.029

L.N. Fletcher, P.G.J. Irwin, N.A. Teanby, G.S. Orton, P.D. Parrish, R. de Kok, C. Howett, S.B. Calcutt, N. Bowles, F.W. Taylor, Characterizing Saturn's vertical temperature structure from Cassini/CIRS. Icarus 189(2), 457-478 (2007b). https://doi.org/10.1016/j.icarus.2007.02.006

L.N. Fletcher, P.G.J. Irwin, G.S. Orton, N.A. Teanby, R.K. Achterberg, G.L. Bjoraker, P.L. Read, A.A. SimonMiller, C. Howett, R. de Kok, N. Bowles, S.B. Calcutt, B. Hesman, F.M. Flasar, Temperature and composition of Saturn's polar hot spots and hexagon. Science 319(5859), 79-81 (2008)

L.N. Fletcher, G.S. Orton, N.A. Teanby, P.G.J. Irwin, Phosphine on Jupiter and Saturn from Cassini/CIRS. Icarus 202, 543-564 (2009a). https://doi.org/10.1016/j.icarus.2009.03.023

L.N. Fletcher, G.S. Orton, N.A. Teanby, P.G.J. Irwin, G.L. Bjoraker, Methane and its isotopologues on Saturn from Cassini/CIRS observations. Icarus 199(2), 351-367 (2009b). https://doi.org/10.1016/j.icarus. 2008.09.019

L.N. Fletcher, R.K. Achterberg, T.K. Greathouse, G.S. Orton, B.J. Conrath, A.A. Simon-Miller, N. Teanby, S. Guerlet, P.G.J. Irwin, F.M. Flasar, Seasonal change on Saturn from Cassini/CIRS observations, 20042009. Icarus 208(1), 337-352 (2010). https://doi.org/10.1016/j.icarus.2010.01.022

L.N. Fletcher, K.H. Baines, T.W. Momary, A.P. Showman, P.G.J. Irwin, G.S. Orton, M. Roos-Serote, C. Merlet, Saturn's tropospheric composition and clouds from Cassini/VIMS 4.6-5.1 $\mu \mathrm{m}$ nightside spectroscopy. Icarus 214(2), 510-533 (2011a). https://doi.org/10.1016/j.icarus.2011.06.006

L.N. Fletcher, B.E. Hesman, P.G.J. Irwin, K.H. Baines, T.W. Momary, A. Sanchez-Lavega, F.M. Flasar, P.L. Read, G.S. Orton, A. Simon-Miller, R. Hueso, G.L. Bjoraker, A. Mamoutkine, T. del Rio-Gaztelurrutia, J.M. Gomez, B. Buratti, R.N. Clark, P.D. Nicholson, C. Sotin, Thermal structure and dynamics of Saturn's northern springtime disturbance. Science 332(6036), 1413-1417 (2011b). https://doi.org/10.1126/ science. 1204774

L.N. Fletcher et al., The origin and evolution of Saturn's 2011-2012 stratospheric vortex. Icarus 221(2), 560586 (2012). https://doi.org/10.1016/j.icarus.2012.08.024

L.N. Fletcher, T.K. Greathouse, G.S. Orton, P.G.J. Irwin, O. Mousis, J.A. Sinclair, R.S. Giles, The origin of nitrogen on Jupiter and Saturn from the 15N/14N ratio. Icarus 238, 170-190 (2014). https://doi.org/10. 1016/j.icarus.2014.05.007

L.N. Fletcher, P.G.J. Irwin, J.A. Sinclair, G.S. Orton, R.S. Giles, J. Hurley, N. Gorius, R.K. Achterberg, B.E. Hesman, G.L. Bjoraker, Seasonal evolution of Saturn's polar temperatures and composition. Icarus 250, 131-153 (2015). https://doi.org/10.1016/j.icarus.2014.11.022

L.N. Fletcher, P.G.J. Irwin, R.K. Achterberg, G.S. Orton, F.M. Flasar, Seasonal variability of Saturn's tropospheric temperatures, winds and para- $\mathrm{H}_{2}$ from Cassini far-IR spectroscopy. Icarus 264, 137-159 (2016). https://doi.org/10.1016/j.icarus.2015.09.009

L.N. Fletcher, S. Guerlet, G.S. Orton, R.G. Cosentino, T. Fouchet, P.G.J. Irwin, L.M. Li, F.M. Flasar, N. Gorius, R. Morales-Juberias, Disruption of Saturn's quasi-periodic equatorial oscillation by the great northern storm. Nat. Astron. 1(11), 765-770 (2017). https://doi.org/10.1038/s41550-017-0271-5

L.N. Fletcher, M. Gustafsson, G.S. Orton, Hydrogen dimers in giant-planet infrared spectra. Astrophys. J. Suppl. Ser. 235, 24 (2018a) 
L.N. Fletcher, G.S. Orton, J.A. Sinclair, S. Guerlet, P.L. Read, A. Antunano, R.K. Achterberg, F.M. Flasar, P.G.J. Irwin, G.L. Bjoraker, J. Hurley, B.E. Hesman, M. Segura, N. Gorius, A. Mamoutkine, S.B. Calcutt, A hexagon in Saturn's northern stratosphere surrounding the emerging summertime polar vortex. Nat. Commun. 9, 3564 (2018b)

L.N. Fletcher, T.K. Greathouse, S. Guerlet, J.I. Moses, R.A. West, Saturn's seasonally changing atmosphere: thermal structure, composition and aerosols, in Saturn in the 21st Century, ed. by K.H. Baines, F.M. Flasar, N. Krupp, T. Stallard (Cambridge University Press, Cambridge, 2018c), pp. 251-294. https:// doi.org/10.1017/9781316227220.010

L.N. Fletcher, Y. Kaspi, T. Guillot, A.P. Showman, How well do we understand the belt/zone circulation of giant planet atmospheres? Space Sci. Rev. 216(2), 30 (2020). https://doi.org/10.1007/s11214-019-0631-9

T. Fouchet, P.G.J. Irwin, P. Parrish, S.B. Calcutt, F.W. Taylor, C.A. Nixon, T. Owen, Search for spatial variation in the Jovian $15 \mathrm{~N} / 14 \mathrm{~N}$ ratio from Cassini/CIRS observations. Icarus 172(1), 50-58 (2004a). https:// doi.org/10.1016/j.icarus.2003.11.011

T. Fouchet, G. Orton, P.G.J. Irwin, S.B. Calcutt, C.A. Nixon, Upper limits on hydrogen halides in Jupiter from Cassini/CIRS observations. Icarus 170, 237-241 (2004b). https://doi.org/10.1016/j.icarus.2004.03.013

T. Fouchet, S. Guerlet, D.F. Strobel, A.A. Simon-Miller, B. Bézard, F.M. Flasar, An equatorial oscillation in Saturn's middle atmosphere. Nature 453(7192), 200-202 (2008). https://doi.org/10.1038/nature06912

T. Fouchet, J.I. Moses, B.J. Conrath, Saturn: composition and chemistry, in Saturn from Cassini-Huygens, ed. by M.K. Doughterty, L.W. Esposito, S.M. Krimigis (Springer, Dordrecht, 2009), pp. 83-112

T. Fouchet, T.K. Greathouse, A. Spiga, L.N. Fletcher, S. Guerlet, J. Leconte, G.S. Orton, Stratospheric aftermath of the 2010 storm on Saturn as observed by the TEXES instrument. I. Temperature structure. Icarus 277, 196-214 (2016). https://doi.org/10.1016/j.icarus.2016.04.030

A.J. Friedson, J.I. Moses, General circulation and transport in Saturn's upper troposphere and stratosphere. Icarus 218(2), 861-875 (2012). https://doi.org/10.1016/j.icarus.2012.02.004

E. Galanti, Y. Kaspi, An adjoint-based method for the inversion of the Juno and Cassini gravity measurements into wind fields. Astrophys. J. 820(2), 91 (2016). https://doi.org/10.3847/0004-637x/820/2/91

E. Galanti, Y. Kaspi, Prediction for the flow-induced gravity field of Saturn: implications for Cassini's Grand Finale. Astrophys. J. Lett. 843, 2 (2017). https://doi.org/10.3847/2041-8213/aa7aec

E. Galanti, Y. Kaspi, E. Tziperman, A full, self-consistent treatment of thermal wind balance on oblate fluid planets. J. Fluid Mech. 810, 175-195 (2017). https://doi.org/10.1017/jfm.2016.687

E. Galanti, Y. Kaspi, Y. Miguel, T. Guillot, D. Durante, P. Racioppa, L. Iess, Saturn's deep atmospheric flows revealed by the Cassini Grand Finale gravity measurements. Geophys. Res. Lett. 46(2), 616-624 (2019). https://doi.org/10.1029/2018GL078087

B. Galperin, R.M.B. Young, S. Sukoriansky, N. Dikovskaya, P.L. Read, A.J. Lancaster, D. Armstrong, Cassini observations reveal a regime of zonostrophic macroturbulence on Jupiter. Icarus 229, 295-320 (2014). https://doi.org/10.1016/j.icarus.2013.08.030

E. Garcia-Melendo, A. Sanchez-Lavega, Shallow water simulations of Saturn's giant storms at different latitudes. Icarus 286, 241-260 (2017). https://doi.org/10.1016/j.icarus.2016.10.006

E. Garcia-Melendo, A. Sanchez-Lavega, R. Hueso, Numerical models of Saturn's long-lived anticyclones. Icarus 191(2), 665-677 (2007). https://doi.org/10.1016/j.icarus.2007.05.020

E. Garcia-Melendo, A. Sanchez-Lavega, J.F. Rojas, S. Perez-Hoyos, R. Hueso, Vertical shears in Saturn's eastward jets at cloud level. Icarus 201(2), 818-820 (2009). https://doi.org/10.1016/j.icarus.2009. 02.022

E. Garcia-Melendo, A. Sanchez-Lavega, J. Legarreta, S. Perez-Hoyos, R. Hueso, A strong high altitude narrow jet detected at Saturn's equator. Geophys. Res. Lett. 37, L22204 (2010). https://doi.org/10.1029/ $2010 \mathrm{~g} 1045434$

E. Garcia-Melendo, J. Arregi, J.F. Rojas, R. Hueso, N. Barrado-Izagirre, J.M. Gomez-Forrellad et al., Dynamics of Jupiter's equatorial region at cloud top level from Cassini and HST images. Icarus 211, 1242-1257 (2011a). https://doi.org/10.1016/j.icarus.2010.11.020

E. Garcia-Melendo, S. Perez-Hoyos, A. Sanchez-Lavega, R. Hueso, Saturn's zonal wind profile in 2004-2009 from Cassini ISS images and its long-term variability. Icarus 215(1), 62-74 (2011b). https://doi.org/10. 1016/j.icarus.2011.07.005

E. Garcia-Melendo, R. Hueso, A. Sanchez-Lavega, J. Legarreta, T. del Rio-Gaztelurrutia, S. Perez-Hoyos, J.F. Sanz-Requena, Atmospheric dynamics of Saturn's 2010 giant storm. Nat. Geosci. 6(7), 525-529 (2013). https://doi.org/10.1038/ngeo1860

J.C. Gerard, B. Hubert, J. Gustin, V.I. Shematovich, D. Bisikalo, G.R. Gladstone, L.W. Esposito, EUV spectroscopy of the Venus dayglow with UVIS on Cassini. Icarus 211, 70-80 (2011). https://doi.org/10. 1016/j.icarus.2010.09.020

J.C. Gerard, J. Gustin, W.R. Pryor, D. Grodent, B. Bonfond, A. Radioti, G.R. Gladstone, J.T. Clarke, J.D. Nichols, Remote sensing of the energy of auroral electrons in Saturn's atmosphere: Hubble and Cassini spectral observations. Icarus 223(1), 211-221 (2013). https://doi.org/10.1016/j.icarus.2012.11.033 
P. Gierasch, B. Conrath, J. Magalhaes, Zonal mean properties of Jupiter's upper troposphere from Voyager infrared observations. Icarus 67, 456-483 (1986)

P.J. Gierasch, A.P. Ingersoll, D. Banfield, S.P. Ewald, P. Helfenstein, A. Simon-Miller, A. Vasavada, H.H. Breneman, D.A. Senske (Galileo Imaging Team), Observation of moist convection in Jupiter's atmosphere. Nature 403(6770), 628-630 (2000). https://doi.org/10.1038/35001017

R.S. Giles, L.N. Fletcher, P.G.J. Irwin, Cloud structure and composition of Jupiter's troposphere from $5 \mu \mathrm{m}$ Cassini VIMS spectroscopy. Icarus 257, 457-470 (2015). https://doi.org/10.1016/j.icarus.2015.05.030

P. Goldreich, D. Keeley, Solar seismology 1. Stability of solar P-modes. Astrophys. J. 211, 934-942 (1977)

T.I. Gombosi, A.P. Ingersoll, Saturn: atmosphere, ionosphere, and magnetosphere. Science 327(5972), 14761479 (2010). https://doi.org/10.1126/science.1179119

S. Guerlet, T. Fouchet, B. Bezard, A.A. Simon-Miller, F.M. Flasar, Vertical and meridional distribution of ethane, acetylene and propane in Saturn's stratosphere from CIRS/Cassini limb observations. Icarus 203, 214-232 (2009). https://doi.org/10.1016/j.icarus.2009.04.002

S. Guerlet, T. Fouchet, B. Bezard, J.I. Moses, L.N. Fletcher, A.A. Simon-Miller, F.M. Flasar, Meridional distribution of $\mathrm{CH}_{3} \mathrm{C}_{2} \mathrm{H}$ and $\mathrm{C}_{4} \mathrm{H}_{2}$ in Saturn's stratosphere from CIRS/Cassini limb and nadir observations. Icarus 209(2), 682-695 (2010). https://doi.org/10.1016/j.icarus.2010.03.033

S. Guerlet, T. Fouchet, B. Bezard, F.M. Flasar, A.A. Simon-Miller, Evolution of the equatorial oscillation in Saturn's stratosphere between 2005 and 2010 from Cassini/CIRS limb data analysis. Geophys. Res. Lett. 38, L09201 (2011). https://doi.org/10.1029/2011g1047192

S. Guerlet et al., Global climate modeling of Saturn's atmosphere. Part I: evaluation of the radiative transfer model. Icarus 238, 110-124 (2014). https://doi.org/10.1016/j.icarus.2014.05.010

S. Guerlet, T. Fouchet, S. Vinatier, A.A. Simon, E. Dartois, A. Spiga, Stratospheric benzene and hydrocarbon aerosols detected in Saturn's auroral regions. Astron. Astrophys. 580, A89 (2015)

S. Guerlet, T. Fouchet, A. Spiga, F.M. Flasar, L.N. Fletcher, B.E. Hesman, N. Gorius, Equatorial oscillation and planetary wave activity in Saturn's stratosphere through the Cassini epoch. J. Geophys. Res., Planets 123(1), 246-261 (2018). https://doi.org/10.1002/2017JE005419

T. Guillot, A comparison of the interiors of Jupiter and Saturn. Planet. Space Sci. 47(10-11), 1183-1200 (1999). https://doi.org/10.1016/s0032-0633(99)00043-4.

T. Guillot, Y. Miguel, B. Militzer, W.B. Hubbard, Y. Kaspi, E. Galanti et al., A suppression of differential rotation in Jupiter's deep interior. Nature 555(7695), 227 (2018). https://doi.org/10.1038/nature25775

J.L. Gunnarson, K.M. Sayanagi, J.J. Blalock, L.N. Fletcher, A.P. Ingersoll, U.A. Dyudina et al., Saturn's new ribbons: Cassini observations of planetary waves in Saturn's 42N atmospheric jet. Geophys. Res. Lett. 45(15), 7399-7408 (2018). https://doi.org/10.1029/2018GL078156

D.A. Gurnett, P. Zarka, R. Manning, W.S. Kurth, G.B. Hospodarsky, T.F. Averkamp et al., Non-detection at Venus of high-frequency radio signals characteristic of terrestrial lightning. Nature 409, 313-315 (2001). https://doi.org/10.1038/35053009

J. Gustin, J.C. Gerard, W. Pryor, P.D. Feldman, D. Grodent, G. Holsclaw, Characteristics of Saturn's polar atmosphere and auroral electrons derived from HST/STIS, FUSE and Cassini/UVIS spectra. Icarus 200(1), 176-187 (2009). https://doi.org/10.1016/j.icarus.2008.11.013

J. Gustin, I. Stewart, J.C. Gerard, L. Esposito, Characteristics of Saturn's FUV airglow from limb-viewing spectra obtained with Cassini-UVIS. Icarus 210(1), 270-283 (2010). https://doi.org/10.1016/j.icarus. 2010.06.031

J. Gustin, D. Grodent, A. Radioti, W. Pryor, L. Lamy, J. Ajello, Statistical study of Saturn's auroral electron properties with Cassini/UVIS FUV spectral images. Icarus 284, 264-283 (2017). https://doi.org/10. 1016/j.icarus.2016.11.017

A. Hadjighasem, G. Haller, Geodesic transport barriers in Jupiter's atmosphere: a video-based analysis. SIAM Rev. 58, 69-89 (2016). https://doi.org/10.1137/140983665

R.A. Hanel, B.J. Conrath, V.G. Kunde, J.C. Pearl, J.A. Pirraglia, Albedo, internal heat flux, and energy balance of Saturn. Icarus 53(2), 262-285 (1983). https://doi.org/10.1016/0019-1035(83)90147-1

T.R. Hanley, P.G. Steffes, B.M. Karpowicz, A new model of the hydrogen and helium-broadened microwave opacity of ammonia based on extensive laboratory measurements. Icarus 202(1), 316-335 (2009). https://doi.org/10.1016/j.icarus.2009.02.002

M.M. Hedman, P.D. Nicholson, Kronoseismology: using density waves in Saturn's C ring to probe the planet's interior. Astron. J. 146, 1 (2013). https://doi.org/10.1088/0004-6256/146/1/12

M.M. Hedman, P.D. Nicholson, More kronoseismology with Saturn's rings. Mon. Not. R. Astron. Soc. 444(2), 1369-1388 (2014). https://doi.org/10.1093/mnras/stu1503

M. Heimpel, J. Aurnou, Turbulent convection in rapidly rotating spherical shells: a model for equatorial and high latitude jets on Jupiter and Saturn. Icarus 187(2), 540-557 (2007). https://doi.org/10.1016/j.icarus. 2006.10.023

M. Heimpel, J. Aurnou, J. Wicht, Simulation of equatorial and high-latitude jets on Jupiter in a deep convection model. Nature 438, 193-196 (2005). https://doi.org/10.1038/nature04208 
M. Heimpel, T. Gastine, J. Wicht, Simulation of deep-seated zonal jets and shallow vortices in gas giant atmospheres. Nat. Geosci. 9, 19 (2016). https://doi.org/10.1038/ngeo2601

R. Helled, T. Guillot, Interior models of Saturn: including the uncertainties in shape and rotation. Astrophys. J. 767(2), 113 (2013). https://doi.org/10.1088/0004-637x/767/2/113

R. Helled, E. Galanti, Y. Kaspi, Saturn's fast spin determined from its gravitational field and oblateness. Nature 520, 202-U140 (2015). https://doi.org/10.1038/nature14278

F. Hersant, D. Gautier, G. Tobie, J.I. Lunine, Interpretation of the carbon abundance in Saturn measured by Cassini. Planet. Space Sci. 56(8), 1103-1111 (2008). https://doi.org/10.1016/j.pss.2008.02.007

B.E. Hesman et al., Saturn's latitudinal $\mathrm{C}_{2} \mathrm{H}_{2}$ and $\mathrm{C}_{2} \mathrm{H}_{6}$ abundance profiles from Cassini/CIRS and groundbased observations. Icarus 202(1), 249-259 (2009). https://doi.org/10.1016/j.icarus.2009.02.013

B.E. Hesman et al., Elusive ethylene detected in Saturn's northern storm region. Astrophys. J. 760(1), 24 (2012). https://doi.org/10.1088/0004-637x/760/1/24

G. Hodosan, C. Helling, R. Asensio-Torres, I. Vorgul, P.B. Rimmer, Lightning climatology of exoplanets and brown dwarfs guided by solar system data. Mon. Not. R. Astron. Soc. 461(4), 3927-3947 (2016). https://doi.org/10.1093/mnras/stw1571

C.J.A. Howett, P.G.J. Irwin, N.A. Teanby, A. Simon-Miller, S.B. Calcutt, L.N. Fletcher, R. de Kok, Meridional variations in stratospheric acetylene and ethane in the southern hemisphere of the Saturnian atmosphere as determined from Cassini/CIRS measurements. Icarus 190(2), 556-572 (2007). https://doi.org/ 10.1016/j.icarus.2007.03.009

W.B. Hubbard, M.K. Dougherty, D. Gautier, R. Jacobson, The interior of Saturn, in Saturn from CassiniHuygens, ed. by M.K. Doughterty, L.W. Esposito, S.M. Krimigis (Springer, Dordrecht, 2009), pp. 7581

B. Hubert, J.C. Gerard, J. Gustin, V.I. Shematovich, D.V. Bisikalo, A.I. Stewart, G.R. Gladstone, UVIS observations of the FUV OI and CO 4P Venus dayglow during the Cassini flyby. Icarus 207, 549-557 (2010). https://doi.org/10.1016/j.icarus.2009.12.029

B. Hubert, J.C. Gerard, J. Gustin, D.V. Bisikalo, V.I. Shematovich, G.R. Gladstone, Cassini-UVIS observation of dayglow FUV emissions of carbon in the thermosphere of Venus. Icarus 220, 635-646 (2012). https://doi.org/10.1016/j.icarus.2012.06.002

V. Hue, T. Cavalie, M. Dobrijevic, F. Hersant, T.K. Greathouse, 2D photochemical modeling of Saturn's stratosphere. Part I: seasonal variation of atmospheric composition without meridional transport. Icarus 257, 163-184 (2015). https://doi.org/10.1016/j.icarus.2015.04.001

V. Hue, T.K. Greathouse, T. Cavalie, M. Dobrijevic, F. Hersant, 2D photochemical modeling of Saturn's stratosphere. Part II: feedback between composition and temperature. Icarus 267, 334-343 (2016). https://doi.org/10.1016/j.icarus.2015.12.007

V. Hue, E. Hersant, T. Cavalie, M. Dobrijevic, J.A. Sinclair, Photochemistry, mixing and transport in Jupiter's stratosphere constrained by Cassini. Icarus 307, 106-123 (2018)

R. Hueso, J. Legarreta, E. Garcia-Melendo, A. Sanchez-Lavega, S. Perez-Hoyos, The Jovian anticyclone BA II. Circulation and interaction with the zonal jets. Icarus 203, 499-515 (2009). https://doi.org/10.1016/ j.icarus.2009.05.004

R. Hueso, J. Legarreta, S. Perez-Hoyos, J.F. Rojas, A. Sanchez-Lavega, A. Morgado, The international outer planets watch atmospheres node database of giant-planet images. Planet. Space Sci. 58(10), 1152-1159 (2010). https://doi.org/10.1016/j.pss.2010.04.006

R. Hueso, A. Sanchez-Lavega, J.F. Rojas, A.A. Simon, T. Barry, T. del Rio-Gaztelurrutia, A. Antunano, K.M. Sayanagi, M. Delcroix, L.N. Fletcher, E. Garcia-Melendo, S. Perez-Hoyos, J. Blalock, F. Colas, J.M. Gomez-Forrellad, J.L. Gunnarson, D. Peach, M.H. Wong, Saturn atmospheric dynamics one year after Cassini: long-lived features and time variations in the drift of the hexagon. Icarus 336, UNSP 113429 (2020)

J. Hurley, P.G.J. Irwin, L.N. Fletcher, J.I. Moses, B. Hesman, J. Sinclair, C. Merlet, Observations of upper tropospheric acetylene on Saturn: no apparent correlation with $2000 \mathrm{~km}$-sized thunderstorms. Planet. Space Sci. 65, 21-37 (2012a). https://doi.org/10.1016/j.pss.2011.12.026

J. Hurley, L.N. Fletcher, P.G.J. Irwin, S.B. Calcutt, J.A. Sinclair, C. Merlet, Latitudinal variation of upper tropospheric $\mathrm{NH}_{3}$ on Saturn derived from Cassini/CIRS far-infrared measurements. Planet. Space Sci. 73(1), 347-363 (2012b). https://doi.org/10.1016/j.pss.2012.08.003

L. Iess, B. Militzer, Y. Kaspi, P. Nicholson, D. Durante, P. Pacioppa, A. Anabtawi, E. Galanti, W. Hubbard, M.J. Mariani, P. Tortora, S. Wahl, M. Zannoni, Measurement and implications of Saturn's gravity field and ring mass. Science (2019). https://doi.org/10.1125/science.aat2965

A.P. Ingersoll, P.J. Gierasch, D. Banfield, A.R. Vasavada (Galileo Imaging Team), Moist convection as an energy source for the large-scale motions in Jupiter's atmosphere. Nature 403(6770), 630-632 (2000). https://doi.org/10.1038/35001021

A.P. Ingersoll, S.P. Ewald, K.M. Sayanagi, J.J. Blalock, Saturn's atmosphere at 1-10 kilometer resolution. Geophys. Res. Lett. 45(15), 7851-7856 (2018). https://doi.org/10.1029/2018GL079255 
W.H. Ip, C.M. Liu, K.C. Pan, Transport and electrodynamical coupling of nano-grains ejected from the Saturnian rings and their possible ionospheric signatures. Icarus 276, 163-169 (2016). https://doi.org/10. 1016/j.icarus.2016.04.004

P.G.J. Irwin, P. Parrish, T. Fouchet, S.B. Calcutt, F.W. Taylor, A.A. Simon-Miller, C.A. Nixon, Retrievals of Jovian tropospheric phosphine from Cassini/CIRS. Icarus 172, 37-49 (2004). https://doi.org/10.1016/j. icarus.2003.09.027

R.A. Jacobson et al., The gravity field of the Saturnian system from satellite observations and spacecraft tracking data. Astron. J. 132(6), 2520-2526 (2006). https://doi.org/10.1086/508812

M.A. Janssen, A.P. Ingersoll, M.D. Allison, S. Gulkis, A.L. Laraia, K.H. Baines, S.G. Edgington, Y.Z. Anderson, K. Kelleher, F.A. Oyafuso, Saturn's thermal emission at 2.2-cm wavelength as imaged by the Cassini RADAR radiometer. Icarus 226(1), 522-535 (2013). https://doi.org/10.1016/j.icarus.2013. 06.008

D.E. Jennings et al., Composite infrared spectrometer (CIRS) on Cassini. Appl. Opt. 56(18), 5274-5294 (2017). https://doi.org/10.1364/ao.56.005274

Y. Kaspi, Inferring the depth of the zonal jets on Jupiter and Saturn from odd gravity harmonics. Geophys. Res. Lett. 40(4), 676-680 (2013). https://doi.org/10.1029/2012g1053873

Y. Kaspi, J.E. Davighi, E. Galanti, W.B. Hubbard, The gravitational signature of internal flows in giant planets: comparing the thermal wind approach with barotropic potential-surface methods. Icarus 276, 170181 (2016). https://doi.org/10.1016/j.icarus.2016.04.001

Y. Kaspi, E. Galanti, W.B. Hubbard, D.J. Stevenson, S.J. Bolton, L. Iess et al., Jupiter's atmospheric jet streams extend thousands of kilometres deep. Nature 555(7695), 223 (2018). https://doi.org/10.1038/ nature 25793

Y. Kaspi, E. Galanti, A.P. Showman, D.J. Stevenson, T. Guillot, L. Iess, S.J. Bolton, Comparison of the deep atmospheric dynamics of Jupiter and Saturn in light of the Juno and Cassini gravity measurements. Space Sci. Rev. 216, 84 (2020)

S.J. Kim, T.R. Geballe, The 2.9-4.2 micron spectrum of Saturn: clouds and $\mathrm{CH}_{4}, \mathrm{PH}_{3}$, and $\mathrm{NH}_{3}$. Icarus 179(2), 449-458 (2005). https://doi.org/10.1016/j.icarus.2005.06.015

S.J. Kim, C.K. Sim, D.W. Lee, R. Courtin, J.I. Moses, Y.C. Minh, The three-micron spectral feature of the Saturnian haze: implications for the haze composition and formation process. Planet. Space Sci. 65 , 122-129 (2012). https://doi.org/10.1016/j.pss.2012.02.013

D. Kong, K. Zhang, G. Schubert, J.D. Anderson, Origin of Jupiter's cloud-level zonal winds remains a puzzle even after Juno. Proc. Natl. Acad. Sci. USA 115(34), 8499-8504 (2018). https://doi.org/10.1073/ pnas. 1805927115

T.T. Koskinen, S. Guerlet, Atmospheric structure and helium abundance on Saturn from Cassini/UVIS and CIRS observations. Icarus 307, 161-171 (2018). https://doi.org/10.1016/j.icarus.2018.02.020

T.T. Koskinen, B.R. Sandel, R.V. Yelle, F.J. Capalbo, G.M. Holsclaw, W.E. McClintock, S. Edgington, The density and temperature structure near the exobase of Saturn from Cassini UVIS solar occultations. Icarus 226(2), 1318-1330 (2013). https://doi.org/10.1016/j.icarus.2013.07.037

T.T. Koskinen, B.R. Sandel, R.V. Yelle, D.F. Strobel, I.C.F. Muller-Wodarg, J.T. Erwin, Saturn's variable thermosphere from Cassini/UVIS occultations. Icarus 260, 174-189 (2015). https://doi.org/10.1016/j. icarus.2015.07.008

T.T. Koskinen, J.I. Moses, R.A. West, S. Guerlet, A. Jouchoux, The detection of benzene in Saturn's upper atmosphere. Geophys. Res. Lett. 43(15), 7895-7901 (2016). https://doi.org/10.1002/2016g1070000

T.T. Koskinen, B.R. Sandel, R. Yelle, G.M. Holsclaw, E. Quemerais, Saturn in Lyman alpha: a comparison of Cassini and Voyager observations. Icarus 339, UNSP 113594 (2020)

V. Lainey, R.A. Jacobson, R. Tajeddine, N.J. Cooper, C. Murray, V. Robert et al., New constraints on Saturn's interior from Cassini astrometric data. Icarus 281, 286-296 (2017). https://doi.org/10.1016/j.icarus. 2016.07.014

L. Lamy, B. Cecconi, R. Prange, P. Zarka, J.D. Nichols, J.T. Clarke, An auroral oval at the footprint of Saturn's kilometric radio sources, colocated with the UV aurorae. J. Geophys. Res. Space Phys. 114, A10212 (2009). https://doi.org/10.1029/2009ja014401

L. Lamy, R. Prange, W. Pryor, J. Gustin, S.V. Badman, H. Melin, T. Stallard, D.G. Mitchell, P.C. Brandt, Multispectral simultaneous diagnosis of Saturn's aurorae throughout a planetary rotation. J. Geophys. Res. Space Phys. 118(8), 4817-4843 (2013). https://doi.org/10.1002/jgra.50404

A.L. Laraia, A.P. Ingersoll, M.A. Janssen, S. Gulkis, F. Oyafuso, M. Allison, Analysis of Saturn's thermal emission at 2.2-cm wavelength: spatial distribution of ammonia vapor. Icarus 226(1), 641-654 (2013). https://doi.org/10.1016/j.icarus.2013.06.017

E. Lellouch, B. Bezard, D.F. Strobel, G.L. Bjoraker, F.M. Flasar, P.N. Romani, On the $\mathrm{HCN}$ and $\mathrm{CO}_{2}$ abundance and distribution in Jupiter's stratosphere. Icarus 184, 478-497 (2006). https://doi.org/10.1016/j. icarus.2006.05.018 
C. Li, A.P. Ingersoll, Moist convection in hydrogen atmospheres and the frequency of Saturn's giant storms. Nat. Geosci. 8(5), 398-403 (2015). https://doi.org/10.1038/ngeo2405

L.M. Li, A.P. Ingersoll, A.R. Vasavada, C.C. Porco, A.D. Del Genio, S.P. Ewald, Life cycles of spots on Jupiter from Cassini images. Icarus 172, 9-23 (2004). https://doi.org/10.1016/j.icarus.2003.10.015

L.M. Li, A.P. Ingersoll, X.L. Huang, Interaction of moist convection with zonal jets on Jupiter and Saturn. Icarus 180, 113-123 (2006a). https://doi.org/10.1016/j.icarus.2005.08.016

L.M. Li, A.P. Ingersoll, A.R. Vasavada, A.A. Simon-Miller, R.K. Achterberg, S.P. Ewald et al., Waves in Jupiter's atmosphere observed by the Cassini ISS and CIRS instruments. Icarus 185, 416-429 (2006b). https://doi.org/10.1016/j.icarus.2006.08.005

L.M. Li, A.P. Ingersoll, A.R. Vasavada, A.A. Simon-Miller, A.D. Del Genio, S.P. Ewald et al., Vertical wind shear on Jupiter from Cassini images. J. Geophys. Res., Planets 111, E04004 (2006c). https://doi.org/ $10.1029 / 2005 \mathrm{je} 002556$

L.M. Li, P.J. Gierasch, R.K. Achterberg, B.J. Conrath, F.M. Flasar, A.R. Vasavada, A.P. Ingersoll, D. Banfield, A.A. Simon-Miller, L.N. Fletcher, Strong jet and a new thermal wave in Saturn's equatorial stratosphere. Geophys. Res. Lett. 35, 23 (2008). https://doi.org/10.1029/2008g1035515

L.M. Li et al., Saturn's emitted power. J. Geophys. Res., Planets 115, E11002 (2010). https://doi.org/10. 1029/2010je003631

L.M. Li et al., Equatorial winds on Saturn and the stratospheric oscillation. Nat. Geosci. 4(11), 750-752 (2011). https://doi.org/10.1038/ngeo1292

L.M. Li, K.H. Baines, M.A. Smith, R.A. West, S. Perez-Hoyos, H.J. Trammell et al., Emitted power of Jupiter based on Cassini CIRS and VIMS observations. J. Geophys. Res., Planets 117, E11002 (2012). https://doi.org/10.1029/2012je004191

L.M. Li et al., Strong temporal variation over one Saturnian year: from Voyager to Cassini. Sci. Rep. 3, 2410 (2013). https://doi.org/10.1038/srep02410

L.M. Li, X. Jiang, H.J. Trammell, Y.F. Pan, J. Hernandez, B.J. Conrath et al., Saturn's giant storm and global radiant energy. Geophys. Res. Lett. 42, 2144-2148 (2015). https://doi.org/10.1002/2015g1063763

L. Li, X. Jiang, R.A. West, P.J. Gierasch, S. Perez-Hoyos, A. Sanchez-Lavega, L.N. Fletcher, J.J. Fortney, B. Knowles, C.C. Porco, K.H. Baines, P.M. Fry, A. Mallama, R.K. Achterberg, A.A. Simon, C.A. Nixon, G.S. Orton, U.A. Dyudina, S.P. Ewald, R.W. Schmude, Less absorbed solar energy and more internal heat for Jupiter. Nat. Commun. 9, 3709 (2018)

M.C. Liang, R.L. Shia, A.Y.T. Lee, M. Allen, A.J. Friedson, Y.L. Yung, Meridional transport in the stratosphere of Jupiter. Astrophys. J. 635, L177-L180 (2005). https://doi.org/10.1086/499624

X.H. Liao, T.H. Feng, K.K. Zhang, On the saturation and temporal variation of mean zonal flows: an implication for equatorial jets on giant planets. Astrophys. J. 666(1), L41-L44 (2007). https://doi.org/10. $1086 / 521526$

B. Little, C.D. Anger, A.P. Ingersoll, A.R. Vasavada, D.A. Senske, H.H. Breneman, W.J. Borucki, S.S.I.T. Galileo, Galileo images of lightning on Jupiter. Icarus 142(2), 306-323 (1999). https://doi.org/10.1006/ icar.1999.6195

J.J. Liu, T. Schneider, Scaling of off-equatorial jets in giant planet atmospheres. J. Atmos. Sci. 72(1), 389-408 (2015). https://doi.org/10.1175/jas-d-13-0391.1

J.J. Liu, T. Schneider, L.N. Fletcher, Constraining the depth of Saturn's zonal winds by measuring thermal and gravitational signals. Icarus 239, 260-272 (2014). https://doi.org/10.1016/j.icarus.2014.05.036

T. Liu, K.M. Sayanagi, S.R. Brueshaber, J.J. Blalock, A.P. Ingersoll, U.A. Dyudina, S.P. Ewald, Saturn's north polar vortex structure extracted from cloud images by the optical flow method. J. Geophys. Res., Planets 124, 3041-3062 (2019)

R.D. Lorenz, C. Elachi, R.D. West, W.T.K. Johnson, M.A. Janssen, M. Moghaddam et al., Cassini Radio Detection and Ranging (RADAR): Earth and Venus observations. J. Geophys. Res. Space Phys. 106, 30271-30279 (2001). https://doi.org/10.1029/2001ja900035

J. Lunine, Chemistry of the solar system revealed in the interiors of the giant planets. Proc. Int. Astron. Union 7(S280), 249-260 (2011). https://doi.org/10.1017/S1743921311025026. The Molecular Universe

C.R. Mankovich, J.J. Fortney, Evidence for a dichotomy in the interior structures of Jupiter and Saturn from helium phase separation. Astrophys. J. 889, 51 (2020)

C. Mankovich, M.S. Marley, J.J. Fortney, N. Movshovitz, Cassini ring seismology as a probe of Saturn's interior. I. Rigid rotation. Astrophys. J. 871(1), 1 (2019). https://doi.org/10.3847/1538-4357/aaf798

S. Markham, D. Stevenson, Excitation mechanisms for Jovian seismic modes. Icarus 306, 200-213 (2018)

K.I. Matcheva, B.J. Conrath, P.J. Gierasch, F.M. Flasar, The cloud structure of the Jovian atmosphere as seen by the Cassini/CIRS experiment. Icarus 179, 432-448 (2005). https://doi.org/10.1016/j.icarus.2005. 06.020

L.C. Mayorga, J. Jackiewicz, K. Rages, R.A. West, B. Knowles, N. Lewis, M.S. Marley, Jupiter's phase variations from Cassini: a testbed for future direct-imaging missions. Astron. J. 152, 209 (2016). https:// doi.org/10.3847/0004-6256/152/6/209 
H. Melin, T. Stallard, S. Miller, J. Gustin, M. Galand, S.V. Badman, W.R. Pryor, J. O’Donoghue, R.H. Brown, K.H. Baines, Simultaneous Cassini VIMS and UVIS observations of Saturn's southern aurora: comparing emissions from $\mathrm{H}, \mathrm{H}_{2}$ and $\mathrm{H}_{3}{ }^{+}$at a high spatial resolution. Geophys. Res. Lett. 38, L15203 (2011). https://doi.org/10.1029/2011g1048457

H. Melin et al., Simultaneous multi-scale and multi-instrument observations of Saturn's aurorae during the 2013 observing campaign. Icarus 263, 56-74 (2016). https://doi.org/10.1016/j.icarus.2015.08.021

B. Militzer, S. Wahl, W.B. Hubbard, Models of Saturn's interior constructed with an accelerated concentric Maclaurin spheroid method. Astrophys. J. 879, 78 (2019)

K.E. Miller, J.H. Waite, R.S. Perryman, M.E. Perry, A. Bouquet, B.A. Magee, B. Bolton, T. Brockwell, M.M. Hedman, C.R. Glein, Cassini INMS constraints on the composition and latitudinal fractionation of Saturn ring rain material. Icarus 339, UNSP 113595 (2020)

R. Morales-Juberias, T.E. Dowling, Jupiter's Great Red Spot: fine-scale matches of model vorticity patterns to prevailing cloud patterns. Icarus 225, 216-227 (2013). https://doi.org/10.1016/j.icarus.2013.03.026

R. Morales-Juberias, K.M. Sayanagi, T.E. Dowling, A.P. Ingersoll, Emergence of polar-jet polygons from jet instabilities in a Saturn model. Icarus 211(2), 1284-1293 (2011). https://doi.org/10.1016/j.icarus.2010. 11.006

R. Morales-Juberias, K.M. Sayanagi, A.A. Simon, L.N. Fletcher, R.G. Cosentino, Meandering shallow atmospheric jet as a model of Saturn's north-polar hexagon. Astrophys. J. Lett. 806, 1 (2015). https://doi.org/ $10.1088 / 2041-8205 / 806 / 1 / 118$

J.I. Moses, T.K. Greathouse, Latitudinal and seasonal models of stratospheric photochemistry on Saturn: comparison with infrared data from IRTF/TEXES. J. Geophys. Res., Planets 110, E09007 (2005)

J.I. Moses, A.R. Poppe, Dust ablation on the giant planets: consequences for stratospheric photochemistry. Icarus 297, 33-58 (2017)

J.I. Moses, B. Bezard, E. Lellouch, G.R. Gladstone, H. Feuchtgruber, M. Allen, Photochemistry of Saturn's atmosphere - I. Hydrocarbon chemistry and comparisons with ISO observations. Icarus 143, 244-298 (2000a). https://doi.org/10.1006/icar.1999.6270

J.I. Moses, E. Lellouch, B. Bezard, G.R. Gladstone, H. Feuchtgruber, M. Allen, Photochemistry of Saturn's atmosphere II. Effects of an influx of external oxygen. Icarus 145(1), 166-202 (2000b). https://doi.org/ 10.1006/icar.1999.6320

J.I. Moses, E.S. Armstrong, L.N. Fletcher, A.J. Friedson, P.G.J. Irwin, J.A. Sinclair, B.E. Hesman, Evolution of stratospheric chemistry in the Saturn storm beacon region. Icarus 261, 149-168 (2015). https://doi. org/10.1016/j.icarus.2015.08.012

O. Mousis, Y. Alibert, W. Benz, Saturn's internal structure and carbon enrichment. Astron. Astrophys. 449(1), 411-415 (2006). https://doi.org/10.1051/0004-6361:20054224

N. Movshovitz, J.J. Fortney, C. Mankovich, D. Thorngren, R. Helled, Saturn's probable interior: an exploration of Saturn's potential interior density structures. Astrophys. J. 891, 109 (2020)

I.C.F. Mueller-Wodarg, T.T. Koskinen, L. Moore, J. Serigano, R.V. Yelle, S. Horst et al., Atmospheric waves and their possible effect on the thermal structure of Saturn's thermosphere. Geophys. Res. Lett. 46(5), 2372-2380 (2019). https://doi.org/10.1029/2018GL081124

A.F. Nagy, A.J. Kliore, M. Mendillo, S. Miller, L. Moore, J.I. Moses, I. Muller-Wodarg, D. Shemansky, Upper atmosphere and ionosphere of Saturn, in Saturn from Cassini-Huygens, ed. by M.K. Doughterty, L.W. Esposito, S.M. Krimigis (Springer, Dordrecht, 2009), pp. 181-201

N. Nettelmann, R. Pustow, R. Redmer, Saturn layered structure and homogeneous evolution models with different EOSs. Icarus 225(1), 548-557 (2013). https://doi.org/10.1016/j.icarus.2013.04.018

C.A. Nixon, R.K. Achterberg, B.J. Conrath, P.G.J. Irwin, N.A. Teanby, T. Fouchet et al., Meridional variations of $\mathrm{C}_{2} \mathrm{H}_{2}$ and $\mathrm{C}_{2} \mathrm{H}_{6}$ in Jupiter's atmosphere from Cassini CIRS infrared spectra. Icarus 188, 47-71 (2007). https://doi.org/10.1016/j.icarus.2006.11.016

C.A. Nixon, R.K. Achterberg, P.N. Romani, M. Allen, X. Zhang, N.A. Teanby et al., Abundances of Jupiter's trace hydrocarbons from Voyager and Cassini. Planet. Space Sci. 58, 1667-1680 (2010). https://doi.org/ 10.1016/j.pss.010.05.008

J. O’Donoghue, T.S. Stallard, H. Melin, G.H. Jones, S.W.H. Cowley, S. Miller et al., The domination of Saturn's low-latitude ionosphere by ring 'rain,'. Nature 496(7444), 193-195 (2013). https://doi.org/10. 1038/nature12049

J. O’Donoghue, T.S. Stallard, H. Melin, S.W.H. Cowley, S.V. Badman, L. Moore, S. Miller, C. Tao, K.H. Baines, J.S.D. Blake, Conjugate observations of Saturn's northern and southern $\mathrm{H}_{3}{ }^{+}$aurorae. Icarus 229, 214-220 (2014). https://doi.org/10.1016/j.icarus.2013.11.009

J. O’Donoghue, L. Moore, J.E.P. Connerney, H. Melin, T.S. Stallard, S. Miller, K.H. Baines, Redetection of the ionospheric $\mathrm{H}_{3}{ }^{+}$signature of Saturn's "ring rain". Geophys. Res. Lett. 44, 11762-11769 (2017). https://doi.org/10.1002/2017gl075932

F. Oliva, A. Adriani, M.L. Moriconi, G.L. Liberti, E. D’Aversa, G. Filacchione, Clouds and hazes vertical structure of a Saturn's giant vortex from Cassini/VIMS-V data analysis. Icarus 278, 215-237 (2016). https://doi.org/10.1016/j.icarus.2016.06.021 
M.E. O’Neill, K.A. Emanuel, G.R. Flierl, Polar vortex formation in giant-planet atmospheres dues to moist convection. Nat. Geosci. 8(7), 523-U118 (2015). https://doi.org/10.1038/ngeo2459

M.E. O'Neill, K.A. Emanuel, G.R. Flierl, Weak jets and strong cyclones: shallow water modeling of giant planet polar caps. J. Atmos. Sci. 73, 1841-1855 (2016). https://doi.org/10.1175/JAS-D-15-0314.1

I. Ordonez-Etxeberria, R. Hueso, A. Sanchez-Lavega, S. Perez-Hoyos, Spatial distribution of Jovian clouds, hazes and colors from Cassini ISS multi-spectral images. Icarus 267, 34-50 (2016). https://doi.org/10. 1016/j.icarus.2015.12.008

G. Orton, A. Friedson, J. Caldwell, H. Hammel, K. Baines, J. Bergstralh, T. Martin, M. Malcom, R. West, W. Golisch, D. Griep, C. Kaminski, A. Tokunaga, R. Baron, M. Shure, Thermal maps of Jupiter - spatialorganization and time-dependence of stratospheric temperatures, 1980 to 1990. Science 252, 537-542 (1991)

G.S. Orton, P.A. Yanamandra-Fisher, B.M. Fisher, A.J. Friedson, P.D. Parrish, J.F. Nelson, A.S. Bauermeister, L. Fletcher, D.Y. Gezari, F. Varosi, A.T. Tokunaga, J. Caldwell, K.H. Baines, J.L. Hora, M.E. Ressler, T. Fujiyoshi, T. Fuse, H. Hagopian, T.Z. Martin, J.T. Bergstralh, C. Howett, W.F. Hoffmann, L.K. Deutsch, J.E. Van Cleve, E. Noe, J.D. Adams, M. Kassis, E. Tollestrup, Semi-annual oscillations in Saturn's low-latitude stratospheric temperatures. Nature 453, 196-199 (2008)

T. Owen, T. Encrenaz, Element abundances and isotope ratios in the giant planets and Titan. Space Sci. Rev. 106(1-4), 121-138 (2003). https://doi.org/10.1023/a:1024633603624.

C.D. Parkinson, A.I.F. Stewart, A.S. Wong, Y.L. Yung, J.M. Ajello, Enhanced transport in the polar mesosphere of Jupiter: evidence from Cassini UVIS helium 584 angstrom airglow. J. Geophys. Res., Planets 111, E02002 (2006). https://doi.org/10.1029/2005je002539

S. Perez-Hoyos, A. Sanchez-Lavega, On the vertical wind shear of Saturn's equatorial jet at cloud level. Icarus 180(1), 161-175 (2006). https://doi.org/10.1016/j.icarus.2005.07.011

S. Perez-Hoyos, A. Sanchez-Lavega, R.G. French, Short-term changes in the belt/zone structure of Saturn's southern hemisphere (1996-2004). Astron. Astrophys. 460(2), 641-645 (2006). https://doi.org/10.1051/ 0004-6361:20065972

S. Perez-Hoyos, J.F. Sanz-Requena, A. Sanchez-Lavega, P.G.J. Irwin, A. Smith, Saturn's tropospheric particles phase function and spatial distribution from Cassini ISS 2010-11 observations. Icarus 277, 1-18 (2016). https://doi.org/10.1016/j.icarus.2016.04.022

M.E. Perry, J.H. Waite, D.G. Mitchell, K.E. Miller, T.E. Cravens, R.S. Perryman, L. Moore, R.V. Yelle, H.-W. Hsu, M.M. Hedman, J.N. Cuzzi, D.F. Strobel, O.Q. Hamil, C.R. Glein, L.J. Paxton, B.D. Teolis, R.L. McNutt, Material flux from the rings of Saturn into its atmosphere. Geophys. Res. Lett. 45, 1009310100 (2018)

J.D.R. Pierel, C.A. Nixon, E. Lellouch, L.N. Fletcher, G.L. Bjoraker, R.K. Achterberg, B. Bezard, B.E. Hesman, P.G.J. Irwin, F.M. Flasar, D/H Ratios on Saturn and Jupiter from Cassini CIRS. Astron. J. 154, 5 (2017). https://doi.org/10.3847/1538-3881/aa899d

C.C. Porco, R.A. West, A. McEwen, A.D. Del Genio, A.P. Ingersoll, P. Thomas et al., Cassini imaging of Jupiter's atmosphere, satellites, and rings. Science 299, 1541-1547 (2003). https://doi.org/10.1126/ science. 1079462

C.C. Porco et al., Cassini imaging science: initial results on Saturn's atmosphere. Science 307(5713), 12431247 (2005). https://doi.org/10.1126/science.1107691

W.R. Pryor et al., The auroral footprint of Enceladus on Saturn. Nature 472(7343), 331-333 (2011). https:// doi.org/10.1038/nature09928

W.R. Pryor, L.W. Esposito, A. Jouchoux, R.A. West, D. Grodent, J.-C. Gerard, A. Radioti, L. Lamy, T. Koskinen, Cassini UVIS detection of Saturn's north polar hexagon in the Grand Finale orbits. J. Geophys. Res., Planets 124, 1979-1988 (2019)

S. Qin, D. Kong, K. Zhang, G. Schubert, Y. Huang, Interpreting the equatorially antisymmetric gravitational field of Saturn measured by the Cassini Grand Finale. Astrophys. J. 890, 26 (2020)

P.L. Read, A chorus of the winds-on Saturn! J. Geophys. Res., Planets 123, 1007-1011 (2018)

P.L. Read, P.J. Gierasch, B.J. Conrath, A. Simon-Miller, T. Fouchet, Y.H. Yamazaki, Mapping potentialvorticity dynamics on Jupiter. I: zonal-mean circulation from Cassini and Voyager 1 data. Q. J. R. Meteorol. Soc. 132, 1577-1603 (2006). https://doi.org/10.1256/qj.05.34

P.L. Read, B.J. Conrath, L.N. Fletcher, P.J. Gierasch, A.A. Simon-Miller, L.C. Zuchowski, Mapping potential vorticity dynamics on Saturn: zonal mean circulation from Cassini and Voyager data. Planet. Space Sci. 57, 1682-1698 (2009a). https://doi.org/10.1016/j.pss.2009.03.004

P.L. Read, T.E. Dowling, G. Schubert, Saturn's rotation period from its atmospheric planetary-wave configuration. Nature 460(7255), 608-610 (2009b). https://doi.org/10.1038/nature08194

J.H. Rogers, H.J. Mettig, A. Cidadao, P.C. Sherrod, D. Peach, Merging circulations on Jupiter: observed differences between cyclonic and anticyclonic mergers. Icarus 185, 244-257 (2006). https://doi.org/10. 1016/j.icarus.2006.05.022 
J.H. Rogers, L.N. Fletcher, G. Adamoli, M. Jacquesson, M. Vedovato, G.S. Orton, A dispersive wave pattern on Jupiter's fastest retrograde jet at $20^{\circ} \mathrm{S}$. Icarus 277, 354-369 (2016). https://doi.org/10.1016/j.icarus. 2016.05.028

M.T. Roman, D. Banfield, P.J. Gierasch, Saturn's cloud structure inferred from Cassini ISS. Icarus 225(1), 93-110 (2013). https://doi.org/10.1016/j.icarus.2013.03.015

M. Rostami, V. Zeitlin, A. Spiga, On the dynamical nature of Saturn's north polar hexagon. Icarus 297, 59-70 (2017)

C. Salyk, A.P. Ingersoll, J. Lorre, A. Vasavada, A.D. Del Genio, Interaction between eddies and mean flow in Jupiter's atmosphere: analysis of Cassini imaging data. Icarus 185, 430-442 (2006). https://doi.org/10. 1016/j.icarus.2006.08.007

A. Sanchez-Lavega, Saturn's Great White Spots, chaos: an interdisciplinary. J. Nonlinear Sci. 4(2), 341-353 (1994). https://doi.org/10.1063/1.166012

A. Sanchez-Lavega, J.F. Rojas, P.V. Sada, P.V. Sada, Saturn's zonal winds at cloud level. Icarus 147(2), 405420 (2000). https://doi.org/10.1006/icar.2000.6449

A. Sanchez-Lavega, R. Hueso, S. Perez-Hoyos, J.F. Rojas, R.G. French, Saturn's cloud morphology and zonal winds before the Cassini encounter. Icarus 170(2), 519-523 (2004). https://doi.org/10.1016/j.icarus. 2004.05.002

A. Sanchez-Lavega, R. Hueso, S. Perez-Hoyos, J.F. Rojas, A strong vortex in Saturn's South Pole. Icarus 184(2), 524-531 (2006). https://doi.org/10.1016/j.icarus.2006.05.020

A. Sanchez-Lavega, R. Hueso, S. Perez-Hoyos, The three-dimensional structure of Saturn's equatorial jet at cloud level. Icarus 187(2), 510-519 (2007). https://doi.org/10.1016/j.icarus.2006.10.022

A. Sanchez-Lavega et al., Deep winds beneath Saturn's upper clouds from a seasonal long-lived planetaryscale storm. Nature 475(7354), 71-74 (2011). https://doi.org/10.1038/nature10203

A. Sanchez-Lavega et al., Ground-based observations of the long-term evolution and death of Saturn's 2010 Great White Spot. Icarus 220(2), 561-576 (2012). https://doi.org/10.1016/j.icarus.2012.05.033

A. Sanchez-Lavega et al., The long- term steady motion of Saturn's hexagon and the stability of its enclosed jet stream under seasonal changes. Geophys. Res. Lett. 41(5), 1425-1431 (2014). https://doi.org/10. 1002/2013g1059078

A. Sanchez-Lavega et al., An enduring rapidly moving storm as a guide to Saturn's equatorial jet's complex structure. Nat. Commun. 7, 13262 (2016). https://doi.org/10.1038/ncomms13262

A. Sánchez-Lavega, G. Fischer, L. Fletcher, E. García-Melendo, B. Hesman, S. Pérez-Hoyos, L. Sromovsky, The great Saturn storm of 2010-2011, in Saturn in the 21st Century, ed. by K. Baines, F. Flasar, N. Krupp, T. Stallard Cambridge Planetary Science (Cambridge University Press, Cambridge, 2018), pp. 377-416. https://doi.org/10.1017/9781316227220.013

A. Sanchez-Lavega, A. Garcia-Munoz, T. del Rio-Gaztelurrutia, S. Perez-Hoyos, J.F. Sanz-Requena, R. Hueso, S. Guerlet, J. Peralta, Multilayer hazes over Saturn's hexagon from Cassini ISS limb images. Nat. Commun. 11, 2281 (2020a)

A. Sanchez-Lavega, E. Garcia-Melendo, J. Legarreta, R. Hueso, T. del Rio-Gaztelurrutia, J.F. Sanz-Requena, S. Perez-Hoyos, A.A. Simon, M.H. Wong, M. Soria, J.M. Gomez-Forrellad, T. Barry, M. Delcroix, K.M. Sayanagi, J.J. Blalock, J.L. Gunnarson, U. Dyudina, S. Ewald, A complex storm system in Saturn's north polar atmosphere in (2018). Nat. Astron. 4, 180-187 (2020b)

J.F. Sanz-Requena, S. Pérez-Hoyos, A. Sánchez-Lavega, A. Antuñano, P.G.J. Irwin, Haze and cloud structure of Saturn's north pole and hexagon wave from Cassini/ISS imaging. Icarus 305, 284-300 (2018). https://doi.org/10.1016/j.icarus.2017.12.043

J.F. Sanz-Requena, S. Pérez-Hoyos, A. Sánchez-Lavega, T. del Rio-Gaztelurrutia, P.G.J. Irwin, Hazes and clouds in a singular triple vortex in Saturn's atmosphere from HST/WFC3 multispectral imaging. Icarus 333, 22-36 (2019). https://doi.org/10.1016/j.icarus.2019.05.037

T.M. Sato, T. Satoh, Y. Kasaba, Retrieval of Jovian cloud structure from the Cassini ISS limb-darkening data I. Continuum scattering phase functions for cloud and haze in the South Tropical Zone. Icarus 222, 100-121 (2013). https://doi.org/10.1016/j.icarus.2012.09.035

K.M. Sayanagi, A.P. Showman, Effects of a large convective storm on Saturn's equatorial jet. Icarus 187(2), 520-539 (2007). https://doi.org/10.1016/j.icarus.2006.10.020

K.M. Sayanagi, R. Morales-Juberias, A.P. Ingersoll, Saturn's northern hemisphere ribbon: simulations and comparison with the meandering Gulf stream. J. Atmos. Sci. 67, 2658-2678 (2010). https://doi.org/ 10.1175/2010jas3315.1

K.M. Sayanagi, U.A. Dyudina, S.P. Ewald, G. Fischer, A.P. Ingersoll, W.S. Kurth, G.D. Muro, C.C. Porco, R.A. West, Dynamics of Saturn's great storm of 2010-2011 from Cassini ISS and RPWS. Icarus 223(1), 460-478 (2013). https://doi.org/10.1016/j.icarus.2012.12.013

K.M. Sayanagi, U.A. Dyudina, S.P. Ewald, G.D. Muro, A.P. Ingersoll, Cassini ISS observation of Saturn's String of Pearls. Icarus 229, 170-180 (2014). https://doi.org/10.1016/j.icarus.2013.10.032 
K.M. Sayanagi, J.J. Blalock, U.A. Dyudina, S.P. Ewald, A.P. Ingersoll, Cassini ISS observation of Saturn's north polar vortex and comparison to the south polar vortex. Icarus $\mathbf{2 8 5}, 68-82$ (2017). https://doi.org/10. 1016/j.icarus.2016.12.011

K.M. Sayanagi, K.H. Baines, U. Dyudina, L.N. Fletcher, A. Sánchez-Lavega, R.A. West, Saturn's polar atmosphere, in Saturn in the 21st Century, ed. by K.H. Baines, F.M. Flasar, N. Krupp, T. Stallard (Cambridge University Press, Cambridge, 2018), pp. 337-376. https://doi.org/10.1017/9781316227220.012

P.J. Schinder, F.M. Flasar, E.A. Marouf, R.G. French, C.A. McGhee, A.J. Kliore, N.J. Rappaport, E. Barbinis, D. Fleischman, A. Anabtawi, Saturn's equatorial oscillation: evidence of descending thermal structure from Cassini radio occultations. Geophys. Res. Lett. 38, L08205 (2011). https://doi.org/10.1029/ $2011 \mathrm{~g} 1047191$

P.J. Schinder, F.M. Flasar, E.A. Marouf, R.G. French, A. Anabtawi, E. Barbinis, A.J. Kliore, A numerical technique for two-way radio occultations by oblate axisymmetric atmospheres with zonal winds. Radio Sci. 50(7), 712-727 (2015). https://doi.org/10.1002/2015rs005690

T. Schneider, J. Liu, Formation of jets and equatorial superrotation on Jupiter. J. Atmos. Sci. 66(3), 579-601 (2009). https://doi.org/10.1175/2008JAS2798.1

D.E. Shemansky, X. Liu, Saturn upper atmospheric structure from Cassini EUV and FUV occultations. Can. J. Phys. 90(8), 817-831 (2012). https://doi.org/10.1139/p2012-036

S. Shetty, P.S. Marcus, Changes in Jupiter's Great Red Spot (1979-2006) and Oval BA (2000-2006). Icarus 210, 182-201 (2010). https://doi.org/10.1016/j.icarus.2010.06.026

A.P. Showman, I. de Pater, Dynamical implications of Jupiter's tropospheric ammonia abundance. Icarus 174(1), 192-204 (2005). https://doi.org/10.1016/j.icarus.2004.10.004

A.P. Showman, A.P. Ingersoll, R. Achterberg, Y. Kaspi, The global atmospheric circulation of Saturn, in Saturn in the 21st Century, ed. by K.H. Baines, F.M. Flasar, N. Krupp, T. Stallard (Cambridge University Press, Cambridge, 2018), pp. 295-336. https://doi.org/10.1017/9781316227220.011

A.A. Simon, L. Li, D.C. Reuter, Small-scale waves on Jupiter: a reanalysis of new horizons, Voyager, and Galileo data. Geophys. Res. Lett. 42(8), 2612-2618 (2015). https://doi.org/10.1002/2015GL063433

A.A. Simon-Miller, P.J. Gierasch, On the long-term variability of Jupiter's winds and brightness as observed from Hubble. Icarus 210, 258-269 (2010). https://doi.org/10.1016/j.icarus.2010.06.020

A.A. Simon-Miller, B.J. Conrath, P.J. Gierasch, G.S. Orton, R.K. Achterberg, F.M. Flasar, B.M. Fisher, Jupiter's atmospheric temperatures: from Voyager IRIS to Cassini CIRS. Icarus 180, 98-112 (2006). https://doi.org/10.1016/j.icarus.2005.07.019

A.A. Simon-Miller, B.W. Poston, G.S. Orton, B. Fisher, Wind variations in Jupiter's equatorial atmosphere: a QQO counterpart? Icarus 186, 192-203 (2007). https://doi.org/10.1016/j.icarus.2006.08.009

A.A. Simon-Miller, J.H. Rogers, P.J. Gierasch, D. Choi, M.D. Allison, G. Adamoli, H.J. Mettig, Longitudinal variation and waves in Jupiter's south equatorial wind jet. Icarus 218, 817-830 (2012). https://doi.org/ 10.1016/j.icarus.2012.01.022

J.A. Sinclair, P.G.J. Irwin, L.N. Fletcher, J.I. Moses, T.K. Greathouse, A.J. Friedson, B. Hesman, J. Hurley, C. Merlet, Seasonal variations of temperature, acetylene and ethane in Saturn's atmosphere from 2005 to 2010, as observed by Cassini-CIRS. Icarus 225(1), 257-271 (2013). https://doi.org/10.1016/j.icarus. 2013.03.011

J.A. Sinclair, P.G.J. Irwin, L.N. Fletcher, T. Greathouse, S. Guerlet, J. Hurley, C. Merlet, From Voyager-IRIS to Cassini-CIRS: interannual variability in Saturn's stratosphere? Icarus 233, 281-292 (2014). https:// doi.org/10.1016/j.icarus.2014.02.009

J.A. Sinclair, G.S. Orton, T.K. Greathouse, L.N. Fletcher, J.I. Moses, V. Hue, P.G.J. Irwin, Jupiter's auroralrelated stratospheric heating and chemistry I: analysis of Voyager-IRIS and Cassini-CIRS spectra. Icarus 292, 182-207 (2017). https://doi.org/10.1016/j.icarus.2016.12.033

J.A. Sinclair, G.S. Orton, T.K. Greathouse, L.N. Fletcher, J.I. Moses, V. Hue, P.G.J. Irwin, Jupiter's auroralrelated stratospheric heating and chemistry II: analysis of IRTF-TEXES spectra measured in December 2014. Icarus 300, 305-326 (2018). https://doi.org/10.1016/j.icarus.2017.09.016

J.A. Sinclair, J.I. Moses, V. Hue, T.K. Greathouse, G.S. Orton, L.N. Fletcher, P.G.J. Irwin, Jupiter's auroralrelated stratospheric heating and chemistry III: abundances of $\mathrm{C}_{2} \mathrm{H}_{4}, \mathrm{CH}_{3} \mathrm{C}_{2} \mathrm{H}, \mathrm{C}_{4} \mathrm{H}_{2}$ and $\mathrm{C}_{6} \mathrm{H}_{6}$ from Voyager-IRIS and Cassini-CIRS. Icarus 328, 176-193 (2019)

B.A. Smith, L. Soderblom, R.M. Batson, P.M. Bridges, J.L. Inge, H. Masursky, E. Shoemaker, R.F. Beebe, J. Boyce, G. Briggs, A. Bunker, S.A. Collins, C. Hansen, T.V. Johnson, J.L. Mitchell, R.J. Terrile, A.F. Cook, J.N. Cuzzi, J.B. Pollack, G.E. Danielson, A.P. Ingersoll, M.E. Davies, G.E. Hunt, D. Morrison, T. Owen, C. Sagan, J. Veverka, R. Strom, V.E. Suomi, A new look at the Saturn system: the Voyager 2 images. Science 215(4532), 504-537 (1982). https://doi.org/10.1126/science.215.4532.504

A. Spiga, S. Guerlet, E. Millour, M. Indurain, Y. Meurdesoif, S. Cabanes, T. Dubos, J. Leconte, A. Boissinot, S. Lebonnois, M. Sylvestre, T. Fouchet, Global climate modeling of Saturn's atmosphere. Part II: multiannual high-resolution dynamical simulations. Icarus 335, UNSP 113377 (2020) 
L.J. Spilker, Cassini's final year at Saturn: science highlights and discoveries. Geophys. Res. Lett. 46, 57545758 (2019)

L.A. Sromovsky, P.M. Fry, The source of widespread $3 \mu \mathrm{m}$ absorption in Jupiter's clouds: constraints from 2000 Cassini VIMS observations. Icarus 210, 230-257 (2010). https://doi.org/10.1016/j.icarus.2010. 06.039

L.A. Sromovsky, P.M. Fry, Composition and structure of fresh ammonia clouds on Jupiter based on quantitative analysis of Galileo/NIMS and New Horizons/LEISA spectra. Icarus 307, 347 (2018)

L.A. Sromovsky, P.M. Fry, Interpretation of shadows and antishadows on Saturn and the evidence against south polar eyewalls. Icarus (2019, in press). https://doi.org/10.1016/j.icarus.2019.113399

L.A. Sromovsky, K.H. Baines, P.M. Fry, Saturn's Great Storm of 2010-2011: evidence for ammonia and water ices from analysis of VIMS spectra. Icarus 226(1), $402-418$ (2013). https://doi.org/10.1016/j. icarus.2013.05.043

L.A. Sromovsky, K.H. Baines, P.M. Fry, T.W. Momary, Cloud clearing in the wake of Saturn's Great Storm of 2010-2011 and suggested new constraints on Saturn's He/H $\mathrm{H}_{2}$ ratio. Icarus 276, 141-162 (2016). https://doi.org/10.1016/j.icarus.2016.04.031

L.A. Sromovsky, K.H. Baines, P.M. Fry, R.W. Carlson, A possibly universal red chromophore for modeling color variations on Jupiter. Icarus 291, 232-244 (2017). https://doi.org/10.1016/j.icarus.2016.12.014

L.A. Sromovsky, K.H. Baines, P.M. Fry, Models of bright storm clouds and related dark ovals in Saturn's Storm Alley as constrained by 2008 Cassini/VIMS spectra. Icarus 302, 360 (2018)

L.A. Sromovsky, K.H. Baines, P.M. Fry, Saturn's south polar cloud composition and structure inferred from 2006 Cassini/VIMS spectra and ISS images. Icarus 344, 113398 (2020). https://doi.org/10.1016/ j.icarus.2019.113398

T.S. Stallard, H. Melin, S. Miller, S.V. Badman, R.H. Brown, K.H. Baines, Peak emission altitude of Saturn's $\mathrm{H}_{3}{ }^{+}$aurora. Geophys. Res. Lett. 39, L15103 (2012a). https://doi.org/10.1029/2012g1052806

T.S. Stallard, H. Melin, S. Miller, J. O’Donoghue, S.W. Cowley, S.V. Badman, A. Adriani, R.H. Brown, K.H. Baines, Temperature changes and energy inputs in giant planet atmospheres: what we are learning from $\mathrm{H}_{3}{ }^{+}$. Philos. Trans. A Math. Phys. Eng. Sci. 370(1978), 5213-5224 (2012b). https://doi.org/10.1098/ rsta.2012.0028

T.S. Stallard, H. Melin, S. Miller, S.V. Badman, K.H. Baines, R.H. Brown et al., Cassini VIMS observations of $\mathrm{H}_{3}{ }^{+}$emission on the nightside of Jupiter. J. Geophys. Res. Space Phys. 120, 6948-6973 (2015). https://doi.org/10.1002/2015ja021097

A. Studwell, L. Li, X. Jiang, K.H. Baines, P.M. Fry, T.W. Momary, U.A. Dyudina, Saturn's global zonal winds explored by Cassini/VIMS 5- $\mu$ m images. Geophys. Res. Lett. 45, 6823-6831 (2018)

K. Sugiyama, K. Nakajima, M. Odaka, K. Kuramoto, Y.Y. Hayashi, Numerical simulations of Jupiter's moist convection layer: structure and dynamics in statistically steady states. Icarus 229, 71-91 (2014). https://doi.org/10.1016/j.icarus.2013.10.016

M.G. Sussman, N.J. Chanover, A.A. Simon-Miller, A.R. Vasavada, R.F. Beebe, Analysis of Jupiter's Oval BA: a streamlined approach. Icarus 210, 202-210 (2010). https://doi.org/10.1016/j.icarus.2010.06.044

M. Sylvestre, S. Guerlet, T. Fouchet, A. Spiga, F.M. Flasar, B. Hesman, G.L. Bjoraker, Seasonal changes in Saturn's stratosphere inferred from Cassini/CIRS limb observations. Icarus 258, 224-238 (2015). https://doi.org/10.1016/j.icarus.2015.05.025

N.A. Teanby, L.N. Fletcher, P.G.J. Irwin, T. Fouchet, G.S. Orton, New upper limits for hydrogen halides on Saturn derived from Cassini-CIRS data. Icarus 185(2), 466-475 (2006). https://doi.org/10.1016/j.icarus. 2006.07.011

V.G. Teifel', V.D. Vdovichenko, P.G. Lysenko, A.M. Karimov, G.A. Kirienko, N.N. Bondarenko et al., Ammonia in Jupiter's atmosphere: spatial and temporal variations of the NH3 absorption bands at 645 and 787 Nm. Sol. Syst. Res. 52(6), 480-494 (2018). https://doi.org/10.1134/S0038094618060072

V.G. Tejfel, Latitudinal variations of the molecular absorption on Saturn and seasonal changes of the atmospheric state at S- and N-hemispheres. Terr. Atmos. Ocean. Sci. 16(1), 231-240 (2005). https://doi.org/ 10.3319/tao.2005.16.1.231(aa)

T. Temma, N.J. Chanover, A.A. Simon-Miller, D.A. Glenar, J.J. Hillman, D.M. Kuehn, Vertical structure modeling of Saturn's equatorial region using high spectral resolution imaging. Icarus 175(2), 464-489 (2005). https://doi.org/10.1016/j.icarus.2004.11.006

H.J. Trammell, L.M. Li, X. Jiang, M. Smith, S. Horst, A. Vasavada, The global vortex analysis of Jupiter and Saturn based on Cassini imaging science subsystem. Icarus 242, 122-129 (2014). https://doi.org/10. 1016/j.icarus.2014.07.019

H.J. Trammell et al., Vortices in Saturn's northern hemisphere (2008-2015) observed by Cassini ISS. J. Geophys. Res., Planets 121(9), 1814-1826 (2016). https://doi.org/10.1002/2016je005122

A.R. Vasavada, S.M. Horst, M.R. Kennedy, A.P. Ingersoll, C.C. Porco, A.D. Del Genio, R.A. West, Cassini imaging of Saturn: southern hemisphere winds and vortices. J. Geophys. Res., Planets 111, E5 (2006). https://doi.org/10.1029/2005je002563 
R.J. Vervack, J.I. Moses, Saturn's upper atmosphere during the Voyager era: reanalysis and modeling of the UVS occultations. Icarus 258, 135-163 (2015). https://doi.org/10.1016/j.icarus.2015.06.007

R.A. West, K.H. Baines, E. Karkoschka, A. Sanchez-Lavega, Clouds and aerosols in Saturn's atmosphere, in Saturn from Cassini-Huygens, ed. by M.K. Doughterty, L.W. Esposito, S.M. Krimigis (Springer, Dordrecht, 2009), pp. 161-179

M.H. Wong, G.L. Bjoraker, M.D. Smith, F.M. Flasar, C.A. Nixon, Identification of the 10- $\mu$ m ammonia ice feature on Jupiter. Planet. Space Sci. 52, 385-395 (2004). https://doi.org/10.1016/j.pss.2003.06.005

M.H. Wong, I. de Pater, X. Asay-Davis, P.S. Marcus, C.Y. Go, Vertical structure of Jupiter's Oval BA before and after it reddened: what changed? Icarus 215, 211-225 (2011). https://doi.org/10.1016/j.icarus. 2011.06.032

R.K. Yadav, J. Bloxham, Deep rotating convection generates the polar hexagon on Saturn. Proc. Natl. Acad. Sci. USA 117, 13991-13996 (2020)

Y. Yair, New results on planetary lightning. Adv. Space Res. 50(3), 293-310 (2012). https://doi.org/10.1016/ j.asr.2012.04.013

Y. Yair, G. Fischer, F. Simoes, N. Renno, P. Zarka, Updated review of planetary atmospheric electricity. Space Sci. Rev. 1371-4, 29-44 (2008). https://doi.org/10.1007/s11214-008-9349-9

S.Y. Ye, G. Fischer, W.S. Kurth, J.D. Menietti, D.A. Gurnett, Rotational modulation of Saturn's radio emissions after equinox. J. Geophys. Res. Space Phys. 121(12), 11714-11728 (2016). https://doi.org/10. 1002/2016ja023281

R.V. Yelle, J. Serigano, T.T. Koskinen, S.M. Horst, M.E. Perry, R.S. Perryman, J.H. Waite, Thermal structure and composition of Saturn's upper atmosphere from Cassini/ion neutral mass spectrometer measurements. Geophys. Res. Lett. 45(20), 10951-10958 (2018). https://doi.org/10.1029/2018GL078454

R.M.B. Young, P.L. Read, Forward and inverse kinetic energy cascades in Jupiter's turbulent weather layer. Nat. Phys. 13, 1135 (2017). https://doi.org/10.1038/nphys4227

A. Youssef, P.S. Marcus, The dynamics of Jovian white ovals from formation to merger. Icarus 162(1), 74-93 (2003). https://doi.org/10.1016/S0019-1035(02)00060-X

P. Zarka, W. Farrell, G. Fischer, A. Konovalenko, Ground-based and space-based radio observations of planetary lightning. Space Sci. Rev. 137(1-4), 257-269 (2008). https://doi.org/10.1007/s11214-008-9366-8

X. Zhang, C.A. Nixon, R.L. Shia, R.A. West, P.G.J. Irwin, R.V. Yelle et al., Radiative forcing of the stratosphere of Jupiter, Part I: atmospheric cooling rates from Voyager to Cassini. Planet. Space Sci. 88, 3-25 (2013a). https://doi.org/10.1016/j.pss.2013.07.005

X. Zhang, R.-L. Shia, Y.L. Yung, Jovian stratosphere as a chemical transport system: benchmark analytical solutions. Astrophys. J. 767, 172 (2013b). https://doi.org/10.1088/0004-637x/767/2/172

X. Zhang, R.A. West, D. Banfield, Y.L. Yung, Stratospheric aerosols on Jupiter from Cassini observations. Icarus 226, 159-171 (2013c). https://doi.org/10.1016/j.icarus.2013.05.020

X. Zhang, R.A. West, P.G.J. Irwin, C.A. Nixon, Y.L. Yung, Aerosol influence on energy balance of the middle atmosphere of Jupiter. Nat. Commun. 6, 10231 (2015). https://doi.org/10.1038/ncomms10231

L.C. Zuchowski, Y.H. Yamazaki, P.L. Read, Modeling Jupiter's cloud bands and decks 1, jet scale meridional circulations. Icarus 200, 548-562 (2009). https://doi.org/10.1016/j.icarus.2008.11.024 